\title{
RATIONAL ISOMORPHISMS BETWEEN $K$-THEORIES AND COHOMOLOGY THEORIES
}

\author{
Eric M. Friedlander and Mark E. Walker
}

\begin{abstract}
The well known isomorphism relating the rational algebraic $K$-theory groups and the rational motivic cohomology groups of a smooth variety over a field of characteristic 0 is shown to be realized by a map (the "Segre map") of infinite loop spaces. Moreover, the associated Chern character map on rational homotopy groups is shown to be a ring isomorphism. A technique is introduced which establishes a useful general criterion for a natural transformation of functors on quasi-projective complex varieties to induce a homotopy equivalence of semi-topological singular complexes. Since semi-topological $K$-theory and morphic cohomology can be formulated as the semi-topological singular complexes associated to $K$-theory and motivic cohomology, this criterion provides a rational isomorphism between the semi-topological $K$-theory groups and the morphic cohomology groups of a smooth complex variety. Consequences include a Riemann-Roch theorem for the Chern character on semitopological $K$-theory and an interpretation of the "topological filtration" on singular cohomology groups in $K$ theoretic terms.
\end{abstract}

\section{INTRODUCTION}

A celebrated consequence of A. Grothendieck's Riemann-Roch Theorem (cf. [SGA6], [BS]) is the fact that the Chern character introduces a multiplicative isomorphism

$$
c h: K_{0}(X) \otimes \mathbb{Q} \stackrel{\cong}{\rightrightarrows} C H^{*}(X) \otimes \mathbb{Q},
$$

where $K_{0}(X)$ denotes the Grothendieck group of algebraic vector bundles on a smooth variety quasiprojective $X$ over a field and $C H^{*}(X)$ denotes the Chow ring of algebraic cycles on $X$ modulo rational equivalence (cf. [F; 15.2.16]). This theorem was extended to higher $K$-groups by S. Bloch [B] (cf. M. Levine [Le]) who established an isomorphism

$$
\bigoplus_{n} K_{n}(X) \otimes \mathbb{Q} \stackrel{\cong}{\rightrightarrows} \bigoplus_{n, q} C H^{q}(X, n) \otimes \mathbb{Q}
$$

relating the Quillen $K$-groups of $X$ to Bloch's higher Chow groups. These results were motivated by the earlier theorem of M. Atiyah and F. Hirzebruch $[\mathrm{AH}]$ which asserts the existence of a multiplicative isomorphism

$$
\operatorname{ch}^{\text {top }}: \bigoplus_{n} K_{\text {top }}^{-n}(T) \otimes \mathbb{Q} \cong \bigoplus_{n, q} H_{\text {sing }}^{2 q-n}(T, \mathbb{Q})
$$

relating the topological (complex) $K$-theory of a space $T$ to its singular cohomology.

Key words and phrases. Chern character, morphic cohomology, motivic cohomology, algebraic $K$-theory, semi-topological $K$-theory.

Both authors were partially supported by the N.S.F. and the N.S.A.

Typeset by $\mathcal{A M}_{\mathcal{M}}-\mathrm{T}_{\mathrm{E}} \mathrm{X}$ 
The purpose of this paper is to further investigate such rational isomorphisms for smooth quasi-projective varieties defined over the complex numbers $\mathbb{C}$ or real number field $\mathbb{R}$. In particular, in Theorem 4.7 we establish a natural isomorphism of graded rings

$$
c h^{s s t}: \bigoplus_{n} K_{n}^{s s t}(X) \otimes \mathbb{Q} \stackrel{\sim}{\longrightarrow} \bigoplus_{n, q} L^{q} H^{2 q-n}(X, \mathbb{Q})
$$

relating the singular semi-topological $K$-theory of a smooth, complex variety to its morphic cohomology. The (singular) semi-topological $K$-theory of complex varieties was introduced and studied by the authors in [FW3] (where it was written $K_{*}\left(X \times \Delta_{t o p}\right)$ ). Morphic cohomology is a theory introduced by the first author and H. B. Lawson based on topological abelian groups of algebraic cycles [FL1]. The isomorphism (0.3) enables us to further our program of establishing the basic formal properties of (singular) semi-topological $K$-theory. Our point of view is that this theory, based as it is on algebraic equivalence of vector bundles, interpolates between rational equivalence and topological equivalence. The analogous program for cohomology led the first author and Lawson to formulate morphic cohomology [FL1], whose properties are well established [F4].

In order to derive (0.3) from (0.1), we first establish that Bloch's isomorphism (0.1) can be realized by a natural transformation of spaces and can be refined to give (as expected) an isomorphism of graded rings induced by the Chern character. We present a simpler proof than the original arguments of Bloch and Levine, one which uses the motivic spectral sequence as constructed by the first author and A. Suslin [FS] following the fundamental construction of Bloch and S. Lichtenbaum [BL]. Theorem 1.10 establishes that the isomorphisms of (0.1) arise as the maps on rational homotopy groups of a natural "Segre map" Seg $: \mathcal{K}(X) \rightarrow \mathcal{H}_{\text {mult }}(X)$ of infinite loop spaces.

In our original formulation [FW2], we defined the semi-topological $K$-theory of a variety $X, K_{*}^{\text {semi }}(X)$, as the homotopy groups of the group completion of the space of algebraic morphisms from $X$ to Grassmannians. We think of this definition as geometric in nature, especially since for a smooth projective curve $C$ it is closely related to a stabilization of the moduli space of stable vector bundles on $C$. Indeed, we achieved several computations in this theory, including the semi-topological $K$-theory of projective curves and homogeneous spaces. However, we have found this formulation of semi-topological $K$-theory inadequate for two reasons: a.) for quasi-projective varieties, we have been unable to provide a clear understanding of the topology on these function spaces; and b.) it appears extremely difficult to prove general properties using this definition.

Consequently, we introduced in [FW3] an alternative formulation, defined by the geometric realization of a certain simplicial space $d \mapsto \mathcal{K}\left(X \times \Delta_{\text {top }}^{d}\right)$, which one can informally describe as the "Kan extension of algebraic $K$-theory from the Zariski site to the analytic site". For projective, weakly normal varieties, the space

$$
\mathcal{K}^{s s t}(X)=\mathcal{K}\left(X \times \Delta_{\text {top }}^{\bullet}\right)=\left|d \mapsto \mathcal{K}\left(X \times \Delta_{\text {top }}^{d}\right)\right|
$$

coincides up to homotopy with $\mathcal{K}^{\text {semi }}(X)[\mathrm{FW} 3 ; 1.4]$. For brevity, we shall typically refer to the homotopy groups of $\mathcal{K}^{s s t}(X)$ as "the semi-topological $K$-groups of $X$ " even in the case in which $X$ is not projective (thus taking $\mathcal{K}^{s s t}(X)$ as our "default" formulation). Since $\mathcal{K}^{s s t}(X)$ is formulated as a sort of colimit of algebraic $K$-theory spaces, we have been able to translate several general properties of algebraic $K$-theory to this theory including mod- $n$ equivalence with algebraic $K$-theory and Nisnevich descent (cf. [FW3]).

The point of view afforded by the formulation $\mathcal{K}^{s s t}(X)$ has led us to develop a very useful recognition principle for establishing isomorphisms of the sort we seek. This recognition principle, Theorem 2.3, is inspired by similar results of V. Voevodsky and uses his techniques. Theorem 2.3 is the result of our many efforts to prove that some version of the Segre map from semi-topological $K$-theory to rational cohomology is a rational equivalence. The problem which continually confronted us is the following: Even though we are primarily interested in the semi-topological $K$-theory of a smooth variety $X$, the colimit involved in the construction of $\mathcal{K}^{s s t}(X)$ necessarily ranges over singular varieties. Hence, results such as Poincaré duality (to name just one of many similar examples) for semi-topological $K$-theory are not easily deduced from their purely algebraic analogues. These difficulties are circumvented with Theorem 2.3 and its corollaries. 
Our recognition principle is employed to relate semi-topological $K$-theory to morphic cohomology. To do this, we verify that morphic cohomology of a smooth variety can be formulated as the homotopy groups of the space

$$
\mathcal{H}_{\text {mult }}^{\text {sst }}(X)=\mathcal{H}_{\text {mult }}\left(X \times \Delta_{\text {top }}^{\bullet}\right)=\left|d \mapsto \mathcal{H}_{\text {mult }}\left(X \times \Delta_{\text {top }}^{d}\right)\right|,
$$

where $\mathcal{H}_{\text {mult }}(Y)$ denotes an (infinite loop) space whose homotopy groups give the motivic cohomology of $Y$ whenever $Y$ is smooth.

For applications, it is important to establish the existence of a graded ring isomorphism as in (0.3). The Chern character ch takes values in rational homotopy groups, but has the great advantage that it is multiplicative. This multiplicative property of the Chern character is not an immediate consequence of our analysis of the Segre map Seg. Indeed, we introduce a technique of realizing homotopy classes of $K$-theory spaces by applying functors to "oriented simplicial complexes" in order to obtain a necessary understanding of the behavior of the Segre map with respect to multiplication in $K$-theory.

The relationship of various theories can best be seen by contemplating the following commutative diagram of (infinite loop) spaces

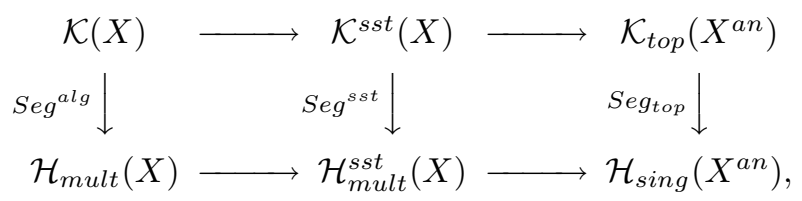

(where $\mathcal{H}_{\text {sing }}$ denotes a space defining singular cohomology). The vertical maps of $(0.4)$ induce isomorphisms on rational homotopy groups equal to the isomorphisms of $(0.1),(0.2)$, and $(0.3)$. The left horizontal maps of (0.4) induce isomorphisms on homotopy groups with finite coefficients [FW3]. As one application of our multiplicative isomorphism (0.3), we conclude in Theorem 5.8 that the right horizontal maps induce isomorphisms in integral homotopy groups provided one inverts the "Bott element" in semi-topological $K$-theory and the "s element" in morphic cohomology. In a forthcoming paper written jointly with C. Haesemeyer [FHW], we will further explore these relationships by establishing spectral sequences of "Atiyah-Hirzebruch type" which relate these algebraic, semi-topological, and topological contexts.

As another consequence of our multiplicative isomorphism (0.3), we conclude that the Chern character of (0.3) satisfies a Riemann-Roch theorem (Theorem 5.2); in other words, not only are the maps of theories associated to (0.4) natural with respect to (contravariant) functoriality, they also satisfy an explicit compatibility with respect to push-forward maps induced by proper maps of varieties.

One intriguing consequence of the multiplicative isomorphism (0.3) is the re-interpretation of the "topological filtration" on the rational singular homology of a projective smooth variety $X$ (as introduced by the first author and B. Mazur in [FM]) in strictly $K$-theoretic terms (cf. Theorem 5.10). Since one expects this filtration to be closely related to the rational Hodge filtration on $H^{*}\left(X^{a n}, \mathbb{Q}\right)$, we hope that this re-interpretation might inject a new point of view into efforts to study the Hodge Conjecture.

Although this paper is almost solely concerned with complex algebraic varieties, we establish the isomorphisms (0.1) for varieties over any field of characteristic 0 and we establish the analogue of isomorphism (0.3) for real varieties (following the consideration of real varieties in [FW4]).

We thank Christian Haesemeyer and Andrei Suslin for sharing their insights concerning $K$-theory and cycles, and we thank Chuck Weibel for helping us straighten out the details of the proof of Proposition 1.7 below. We also acknowledge the impetus this work received from preprints of Ralph Cohen and Paulo Lima-Filho [CL1], [CL2] in which a mistaken proof of (0.3) is given.

\section{$\S 1$ Algebraic $K$-theory, Motivic cohomology, and Chern classes}

In this section, we recall and study the construction of the total Segre class map (originally developed in [FW2])

$$
\text { Seg }: \mathcal{K}^{\text {naive }}(X) \rightarrow \mathcal{H}_{\text {mult }}(X)
$$


from (naive) algebraic $K$-theory (see Definition 1.1) to motivic cohomology. Using the rational degeneration of the motivic spectral sequence (cf. [FS], [Le]) and a "dimension shifting" argument, we prove in Theorem 1.10 that $S e g$ is a rational equivalence for smooth varieties. Moreover, in Theorem 1.12, we show for smooth varieties that the Chern character

$$
c h: \bigoplus_{n} K_{n}(X)_{\mathbb{Q}} \stackrel{\cong}{\longrightarrow} \bigoplus_{n, q} H_{\mathcal{M}}^{2 q-n}(X, \mathbb{Q}(q)) .
$$

is an isomorphism of graded rings.

As seen in Proposition 1.5, it is not difficult to determine the effect of $S e g$ on $\pi_{0}$ groups. Essentially, this is the classical total Segre class, closely related to the total Chern class. Using Riemann-Roch, we can relate $S e g_{0}=\pi_{0} S e g$ to the maps on $\pi_{0}$ induced by the tower of maps in the Friedlander-Suslin tower of spaces determining the motivic spectral sequence.

Unlike the remainder of the paper, this section is purely algebraic in nature, and thus we will be working in the category $S c h / k$ of quasi-projective varieties defined over an arbitrary base field $k$ of characteristic 0 . (We need the hypothesis of characteristic 0 so that resolutions of singularities is known to hold, and hence so that "naive" motivic cohomology coincides with motivic cohomology defined by Voevodsky.)

We now recall the construction of the total Segre class map from [FW2]. Let Grass denote the ind-variety $\varliminf_{N} \operatorname{Grass}\left(k^{N}\right)$ where $\operatorname{Grass}\left(k^{N}\right)=\coprod_{r} \operatorname{Grass}_{r}\left(k^{N}\right)$. Here, $\operatorname{Grass}_{r}\left(k^{N}\right)$ parameterizes all $r$ dimensional quotient $k$-vector spaces of $k^{N}$ and the transition map $\operatorname{Grass}_{r}\left(k^{N}\right) \rightarrow \operatorname{Grass}_{r}\left(k^{N+1}\right)$ sends the quotient $k^{N} \rightarrow V$ to the quotient determined by the composition of $k^{N+1} \rightarrow k^{N} \rightarrow V$. By definition, Hom(-, Grass) is the functor sending $X$ to $\varliminf_{N} \operatorname{Hom}\left(X, \operatorname{Grass}\left(k^{N}\right)\right)$. As in [FW2], we define $\mathcal{I}\left(\Delta^{\bullet}\right)$ to be the simplicial $E_{\infty}$ operad $n \mapsto \mathcal{I}(n)\left(\Delta^{\bullet}\right)$, where $\mathcal{I}(n)\left(\Delta^{d}\right)$ consists of the set of $\Gamma\left(\Delta^{d}\right)$-linear injections of $\left(\Gamma\left(\Delta^{d}\right)^{\infty}\right)^{n}$ into $\Gamma\left(\Delta^{d}\right)^{\infty}$. (Here, $\Gamma\left(\Delta^{d}\right)=k\left[x_{0}, \ldots, x_{d}\right] /\left(\sum_{i} x_{i}=1\right)$.) By $\mathcal{I}$, we mean the associated topological $E_{\infty}$ operad $n \mapsto\left|\mathcal{I}(n)\left(\Delta^{\bullet}\right)\right|$. For any quasi-projective $k$-variety $X$, the simplicial set $\operatorname{Hom}\left(X \times \Delta^{\bullet}\right.$, Grass $)$ admits the structure of an $\mathcal{I}\left(\Delta^{\bullet}\right)$-space, and thus $\mid \operatorname{Hom}\left(X \times \Delta^{\bullet}\right.$, Grass) $\mid$ is an $\mathcal{I}$-space (cf. [FW2;6.8]).

The total Segre class map, as defined in [FW2], arises from the collection of morphisms $\operatorname{Grass}_{r}\left(k^{N}\right) \rightarrow$ $\mathcal{C}_{r-1}\left(\mathbb{P}^{N-1}\right)$, where $\mathcal{C}_{r-1}\left(\mathbb{P}^{N-1}\right)$ denotes the Chow monoid of effective $r$-cycles on $\mathbb{P}^{N-1}$. (See the beginning of $\S 3$ for a brief discussion of Chow varieties.) These morphisms are defined by sending a quotient $k^{N} \rightarrow V$ to the cycle associated to the closed subscheme $\mathbb{P}(V) \subset \mathbb{P}^{N-1}$, where in general $\mathbb{P}(W)$ is defined to be the projective variety associated to the symmetric algebra $S_{k}^{\bullet} W$. To accommodate the case $r=0$, we define $\mathcal{C}_{-1}\left(\mathbb{P}^{M}\right)$ to be $\coprod_{\mathbb{N}}$ Spec $k$, and we think of $\mathcal{C}_{-1}\left(\mathbb{P}^{M}\right)$ as the free abelian monoid scheme on the "empty cycle" which corresponds to the copy of Spec $k$ indexed by $1 \in \mathbb{N}$. The morphism $\operatorname{Spec} k=\operatorname{Grass}_{0}\left(k^{N}\right) \rightarrow \mathcal{C}_{-1}\left(\mathbb{P}^{N-1}\right)$ is then defined by sending $k^{N} \rightarrow 0$ to the empty cycle.

Let Chow denote the ind-variety $\varliminf_{N} \coprod_{r \geq 0} \mathcal{C}_{r-1} \mathbb{P}^{N}$ and let Hom(-, Chow $)^{+}$denote the functor sending a connected variety $X$ to $\varliminf_{N} \bigsqcup_{r \geq 0} \operatorname{Hom}\left(X, \mathcal{C}_{r-1}\left(\mathbb{P}^{N}\right)\right)^{+}$, where the superscript + denotes taking the group completion of the displayed abelian monoid. The value of $\operatorname{Hom}(-, \text { Chow })^{+}$on a general $X$ is given by $\bigoplus_{i=1}^{k} \operatorname{Hom}\left(X_{i}, \text { Chow }\right)^{+}$, where $X=\coprod_{i=1}^{k} X_{i}$ is a decomposition of $X$ into its connected components.

The morphisms $\operatorname{Grass}_{r}\left(\mathbb{C}^{N}\right) \rightarrow \mathcal{C}_{r-1}\left(\mathbb{P}^{N-1}\right)$ stabilizes to produce a natural transformation

$$
\operatorname{Hom}(-, \text { Grass }) \rightarrow \operatorname{Hom}(-, \text { Chow })^{+}
$$

which factors through

$$
\operatorname{Hom}(X, \operatorname{Grass}) \rightarrow \coprod_{r \geq 0} \operatorname{Hom}\left(X, \mathcal{C}_{r-1}\left(\mathbb{P}^{\infty}\right)\right)_{1}^{+}
$$

Here, the subscript 1 designates the subset of $\operatorname{Hom}\left(X, \mathcal{C}_{r-1}\left(\mathbb{P}^{\infty}\right)\right)^{+}$consisting of cycles which have degree 1 on each connected component of $X$. (By convention, the empty cycle has degree 1.) For brevity, we write 
$\operatorname{Hom}(X, \text { Chow })_{1}^{+}$for $\coprod_{r \geq 0} \operatorname{Hom}\left(X, \mathcal{C}_{r-1}\left(\mathbb{P}^{\infty}\right)\right)_{1}^{+}$. We thus have a natural transformation of functors from $S c h / k$ to Sets:

$$
\text { cyc }: \operatorname{Hom}(-, \text { Grass }) \rightarrow \operatorname{Hom}(-, \text { Chow })_{1}^{+} .
$$

As shown in $[\mathrm{FW} 2 ; 6.9]$, for any $X$ the simplicial set

$$
\operatorname{Hom}\left(X \times \Delta^{\bullet}, \text { Chow }\right)_{1}^{+}
$$

admits the structure of an $\mathcal{I}\left(\Delta^{\bullet}\right)$-space given by taking linear join of cycles such that the map

$$
\text { cyc }: \operatorname{Hom}\left(\Delta^{\bullet}, \text { Grass }\right) \rightarrow \operatorname{Hom}\left(\Delta^{\bullet}, \text { Chow }\right)_{1}^{+}
$$

is a map of $\mathcal{I}\left(\Delta^{\bullet}\right)$-spaces.

Recall that $z_{\text {equi }}\left(\mathbb{A}^{q}, 0\right)(-)$ is the presheaf (in fact, $q f h$ sheaf) of abelian groups defined on $S c h / k$ whose value at a normal variety $U$ is the collection of cycles in $U \times \mathbb{A}^{q}$ which are quasi-finite and dominant over some component of $U$. (See section 3 for a more detailed definition.) The following definitions introduce the main objects of study for this section.

Definition 1.1. Let $X$ be a quasi-projective $k$-variety. Define $\mathcal{K}^{\text {naive }}(X)$ to be the spectrum associated (as in [Ma; §14] $)$ to the $\mathcal{I}$-space $\left|\operatorname{Hom}\left(X \times \Delta^{\bullet}, \mathrm{Grass}\right)\right|$. Define $\mathcal{H}_{\text {mult }}(X)$ to be the spectrum associated to the $\mathcal{I}$-space $\mid \operatorname{Hom}\left(X \times \Delta^{\bullet} \text {, Chow }\right)_{1}^{+} \mid$. We also write, ambiguously, $\mathcal{K}^{\text {naive }}(X)$ and $\mathcal{H}_{\text {mult }}(X)$ for the group-like $H$-spaces given by taking zeroth spaces of these spectra. The map

$$
\operatorname{Seg}: \mathcal{K}^{\text {naive }}(X) \rightarrow \mathcal{H}_{\text {mult }}(X)
$$

is defined as the map of spectra (or group-like $H$-spaces) associated to the map of $\mathcal{I}$-spaces

$$
\text { cyc }:\left|\operatorname{Hom}\left(X \times \Delta^{\bullet}, \operatorname{Grass}\right)\right| \rightarrow \mid \operatorname{Hom}\left(X \times \Delta^{\bullet}, \text { Chow }\right)_{1}^{+} \mid .
$$

For all $q \geq 0$, define $\mathcal{H}_{\mathcal{M} \text {, naive }}(X, \mathbb{Z}(q))$ to be the abelian topological group obtained by taking the geometric realization of the simplicial abelian group $d \mapsto z_{\text {equi }}\left(\mathbb{A}^{q}, 0\right)\left(X \times \Delta^{d}\right)$,

$$
\mathcal{H}_{\mathcal{M}, \text { naive }}(X, \mathbb{Z}(q))=\left|d \mapsto z_{\text {equi }}\left(\mathbb{A}^{q}, 0\right)\left(X \times \Delta^{d}\right)\right| .
$$

The homotopy groups for each of these constructions are denoted as follows:

$$
\begin{aligned}
\pi_{n} \mathcal{K}^{\text {naive }}(X) & =K_{n}^{\text {naive }}(X) \\
\pi_{n} \mathcal{H}_{\text {mult }}(X) & =H_{\text {mult }}^{-n}(X) \\
\pi_{n} \mathcal{H}_{\mathcal{M}, \text { naive }}(X, \mathbb{Z}(q)) & =H_{\mathcal{M}, \text { naive }}^{2 q-n}(X, \mathbb{Z}(q)) .
\end{aligned}
$$

Proposition 1.2. Suppose $X$ is a smooth, quasi-projective $k$-variety. Then there is a chain of natural homotopy equivalences joining the spectrum $\mathcal{K}^{\text {naive }}(X)$ and $\mathcal{K}(X)=\Omega B Q \mathcal{P}(X)$, the algebraic $K$-theory spectrum defined from the category of locally free $\mathcal{O}_{X}$-modules using Quillen's $Q$ construction.

Proof. This follows from [GW; 3.3]. See also [FW2; 6.8].

Recall that for a variety $X$, the simplicial abelian group $\mathcal{Z}^{d}(X, \bullet)$ consists in degree $n$ of the group of codimension $d$ cycles on $X \times \Delta^{n}$ which meet the faces of $\Delta^{n}$ properly. The group $C H^{d}(X, n)$ is by definition the $n$-th homotopy group of this simplicial object. 
Theorem 1.3. [Suslin] Let $X$ be a smooth, quasi-projective variety defined over a field $k$ of characteristic zero. Then there are natural isomorphisms

$$
H_{\mathcal{M}, \text { naive }}^{2 q-n}(X, \mathbb{Z}(q)) \cong H_{\mathcal{M}}^{2 q-n}(X, \mathbb{Z}(q)) \cong C H^{q}(X, n),
$$

where $H_{\mathcal{M}}$ denotes the motivic groups as defined using the cdh-hypercohomology of the complexes

$$
z_{\text {equi }}\left(\mathbb{A}^{q}, 0\right)\left(-\times \Delta^{\bullet}\right), \quad q \geq 0
$$

(cf. [FV; 9.2]).

Proof. This follows from [FV; 4.4 and 8.1] and [Su; 2.1].

In light of Proposition 1.2 and Theorem 1.3, if $X$ is a smooth, quasi-projective $k$-variety (with char $k=0$ ), we usually suppress the superscript "naive" and simply write $\mathcal{K}(X)$ for $\mathcal{K}^{\text {naive }}(X)$ and $\mathcal{H}_{\mathcal{M}}(X, \mathbb{Z}(q))$ for $\mathcal{H}_{\mathcal{M}, \text { naive }}(X, \mathbb{Z}(q))$.

In [FW2], the space $\mathcal{H}_{\text {mult }}(X)$ is related explicitly to the spaces $\mathcal{H}_{\mathcal{M} \text {,naive }}(X, \mathbb{Z}(q))$. However, the construction of [FW2] involves choosing inverses to certain homotopy equivalences, which makes the type of dimension shifting argument we employ in this paper impossible. Thus we refine slightly the results of [FW2] with the following proposition. Note that there is a natural transformation

$$
\mid \operatorname{Hom}\left(X \times \Delta^{\bullet}, \text { Chow }\right)_{1}^{+} \mid \rightarrow \operatorname{Hom}(X, \mathbb{N})
$$

of $\mathcal{I}$-spaces induced by sending $\mathcal{C}_{r-1}\left(\mathbb{P}^{\infty}\right)$ to $r-1 \in \mathbb{N}$. Here, the structure of $\operatorname{Hom}(X, \mathbb{N})$ as an $\mathcal{I}$-space is the standard one for an abelian monoid, and the associated group-like $H$-space is naturally equivalent to $\operatorname{Hom}(X, \mathbb{Z})$, so that we obtain a natural map

$$
\mathcal{H}_{\text {mult }}(X) \rightarrow \operatorname{Hom}(X, \mathbb{Z})
$$

Proposition 1.4. For any quasi-projective $k$-variety $X$, the sequence of natural transformations

$$
\left|\operatorname{Hom}\left(X \times \Delta^{\bullet}, \mathcal{C}_{0}\left(\mathbb{P}^{\infty}\right)\right)_{1}^{+}\right| \rightarrow \mathcal{H}_{m u l t}(X) \rightarrow \operatorname{Hom}(X, \mathbb{Z}),
$$

identifies $\left|\operatorname{Hom}\left(X \times \Delta^{\bullet}, \mathcal{C}_{0} \mathbb{P}^{\infty}\right)_{1}^{+}\right|$as the homotopy fiber over $(1, \ldots, 1) \in \operatorname{Hom}(X, \mathbb{Z})$. where the first map is given as the composition of $\left|\operatorname{Hom}\left(X \times \Delta^{\bullet}, \mathcal{C}_{0}\left(\mathbb{P}^{\infty}\right)\right)_{1}^{+}\right| \hookrightarrow \mid \operatorname{Hom}\left(X \times \Delta^{\bullet}, \text { Chow }\right)_{1}^{+} \mid \rightarrow \mathcal{H}_{\text {mult }}(X)$. Moreover, there is a natural homotopy equivalence of the form

$$
\operatorname{Hom}\left(X \times \Delta^{\bullet}, \mathcal{C}_{0}\left(\mathbb{P}^{\infty}\right)\right)_{1}^{+} \stackrel{\sim}{\longrightarrow}\{1\} \times \varliminf_{N} \prod_{q=1}^{N} \mathcal{H}_{\mathcal{M}, \text { naive }}(X, \mathbb{Z}(q)) .
$$

In particular, there is a weak equivalence

$$
\mathcal{H}_{\text {mult }}(X) \stackrel{\sim}{\longrightarrow} \operatorname{Hom}(X, \mathbb{Z}) \times\left(\{1\} \times \underline{\lim _{N}} \prod_{q=1}^{N} \mathcal{H}_{\mathcal{M}, \text { naive }}(X, \mathbb{Z}(q))\right)
$$

which is natural up to homotopy.

Proof. We may assume $X$ is connected. Regard $\mathcal{H}_{\text {mult }}(X)$ as a pointed space with base point determined by the image of the constant map with target $\mathbb{P}^{0}$ under $\operatorname{Hom}(X, \text { Chow })_{1}^{+} \rightarrow \mathcal{H}_{\text {mult }}(X)$ and regard $\mathbb{Z}$ as a 
pointed space with 1 serving as the base point. Then the displayed sequence is a sequence of pointed maps and is clearly natural in $X$. Moreover, the composite map

$$
\left|\operatorname{Hom}\left(X \times \Delta^{\bullet}, \mathcal{C}_{0}\left(\mathbb{P}^{\infty}\right)\right)_{1}^{+}\right| \rightarrow \operatorname{Hom}(X, \mathbb{Z})=\mathbb{Z}
$$

is clearly the constant map, and so there exists a natural map from $\operatorname{Hom}\left(X \times \Delta^{\bullet}, \mathcal{C}_{0}\left(\mathbb{P}^{\infty}\right)\right)_{1}^{+}$to the homotopy fiber of $\mathcal{H}_{\text {mult }}(X) \rightarrow \mathbb{Z}$ over 1 . To establish the first assertion, it suffices to prove this map is a homotopy equivalence.

One can repeat the proof of [FW4; 7.3] using the operad $\mathcal{I}$ in place of the operad $\mathcal{L}$ used in that paper and using the geometric realization of the simplicial group $\mathrm{GL}_{n}\left(\Delta_{k}^{\bullet}\right)$ in place of the topological group $\mathrm{GL}_{n}(\mathbb{R})$. One thereby proves that there is a homotopy equivalence from the mapping telescope $T$ of the infinite sequence of pointed space maps

$$
\mid \operatorname{Hom}\left(X \times \Delta^{\bullet}, \text { Chow }\right)_{1}^{+}|\stackrel{\alpha}{\longrightarrow}| \operatorname{Hom}\left(X \times \Delta^{\bullet}, \text { Chow }\right)_{1}^{+} \mid \stackrel{\alpha}{\longrightarrow} \cdots,
$$

to $\mathcal{H}_{\text {mult }}(X)$, where $\alpha$ is defined by suspension. Moreover, the composition of $T \rightarrow \mathcal{H}_{\text {mult }}(X) \rightarrow \mathbb{Z}$ is induced by the ladder of maps

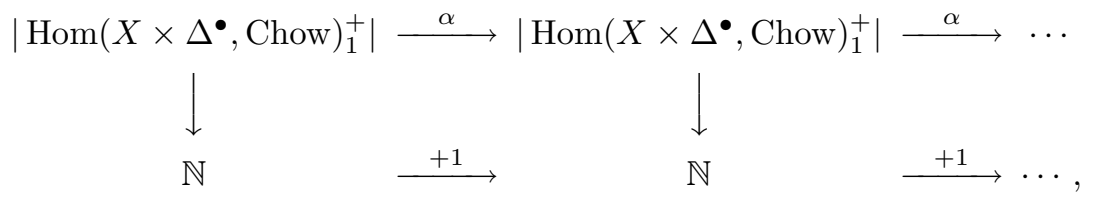

and thus the fiber of $T \rightarrow \mathbb{Z}$ over 1 is given by the mapping telescope of

$$
\left|\operatorname{Hom}\left(X \times \Delta^{\bullet}, \mathcal{C}_{0} \mathbb{P}^{\infty}\right)_{1}^{+}\right| \stackrel{\alpha}{\longrightarrow}\left|\operatorname{Hom}\left(X \times \Delta^{\bullet}, \mathcal{C}_{1} \mathbb{P}^{\infty}\right)_{1}^{+}\right| \stackrel{\alpha}{\longrightarrow} \cdots .
$$

Each map in this sequence is actually a homotopy equivalence by the Lawson suspension theorem $[\mathrm{L}]$, and thus $\left|\operatorname{Hom}\left(X \times \Delta^{\bullet}, \mathcal{C}_{0} \mathbb{P}^{\infty}\right)_{1}^{+}\right|$is homotopy equivalent to the fiber of $T$ over 1 .

The natural homotopy equivalence (1.4.1) is proven in [FW-2; 6.5]; observe that the map defined there is strictly natural in $X$.

The final assertion follows by choosing a splitting for the above sequence of group-like $H$-spaces. Note that such a choice of splitting results in a loss of strict functoriality, but that functoriality up to homotopy is preserved.

Proposition 1.4 gives, for each $n \geq 0$, a natural isomorphism of abelian groups

$$
\pi_{n} \mathcal{H}_{\text {mult }}(X) \cong \pi_{n} \operatorname{Hom}(X, \mathbb{Z}) \times\left(\{1\} \times \bigoplus_{q=1}^{\infty} H_{\mathcal{M}, \text { naive }}^{2 q-n}(X, \mathbb{Z}(n))\right),
$$

where the group law for the the second factor is given by join of cycles. (Here, $\operatorname{Hom}(X, \mathbb{Z})$ is regarded as a discrete topological group.) We define

$$
\operatorname{Seg}_{n}: K_{n}^{\text {naive }}(X) \rightarrow H_{\mathcal{M}}^{-n}(X, \mathbb{Z}(0)) \times\left(\{1\} \times \bigoplus_{q \geq 1} H_{\mathcal{M}, \text { naive }}^{2 q-n}(X, \mathbb{Z}(q))\right)
$$

to be the composition of the map $\pi_{n} S e g: K_{n}^{\text {naive }}(X) \rightarrow \pi_{n} \mathcal{H}_{\text {mult }}(X)$ with the isomorphism of Proposition 1.4. We define $S e g_{n, q}$ to be the $q$-th component of $S e g_{n}$, defined by composing $S e g_{n}$ with projection onto the summand $H_{\mathcal{M}, \text { naive }}^{2 q-n}(X, \mathbb{Z}(q))$. Observe that $\operatorname{Seg}_{0,0}: K_{0}^{\text {naive }}(X) \rightarrow \operatorname{Hom}(X, \mathbb{Z})$ equals $\operatorname{rank}(-)$, the map which gives the multi-rank of a vector bundle on $X$.

We recall that the (classical) total Segre class $s(E)=1+s_{1}(E)+\cdots+s_{i}(E)+\cdots$ is defined by the formula $s(E)=1 / c(E)$, where $c(E) \in\left(\{1\} \times \bigoplus_{q \geq 1} C H^{q}(X)\right)^{\times}$is the total Chern class of a vector bundle $E$. 
Proposition 1.5. If $X$ is a smooth, quasi-projective $k$-variety and $x$ is an element of $K_{0}(X)$, then under the isomorphism $H_{\mathcal{M}}^{2 q}(X, \mathbb{Z}(q)) \cong C H^{q}(X)$ of Theorem 1.3 we have

$$
\operatorname{Seg}_{0}(x)=\operatorname{rank}(x)+c\left(x^{*}\right)^{-1} \in C H^{*}(X),
$$

where $c(-)$ denotes the classical total Chern class map with values in the multiplicative group

$$
\left(\{1\} \times \bigoplus_{q \geq 1} C H^{q}(X)\right)^{\times},
$$

and $x^{*}$ denotes the dual of $x$. Thus, for $q \geq 1$, we have that Seg $g_{0, q}$ coincides with $(-1)^{q}$ times the classical $q$-th Segre class map,

$$
\operatorname{Seg}_{0, q}=(-1)^{q} s_{q}, \quad q \geq 1 \quad \text { and } \quad \operatorname{Seg} g_{0,0}=\operatorname{rank}(-) .
$$

In particular, for such $X$ the map $S_{0} g_{0}$ induces an isomorphism after tensoring with $\mathbb{Q}$ :

$$
\begin{aligned}
\operatorname{Seg}_{0}: K_{0}(X)_{\mathbb{Q}} \cong \pi_{0} \mathcal{H}_{\text {mult }}(X)_{\mathbb{Q}} & \cong H_{\mathcal{M}}^{0}(X, \mathbb{Q}(0)) \oplus\left(\{1\} \times \bigoplus_{q \geq 1} H_{\mathcal{M}}^{2 q}(X, \mathbb{Q}(q))\right)^{\times} \\
& \cong C H^{0}(X) \times\left(\{1\} \times \bigoplus_{q \geq 1} C H^{q}(X)\right)^{\times} .
\end{aligned}
$$

Proof. The map $\operatorname{Seg}_{0,0}: K_{0}(X) \rightarrow \mathbb{Z}$ is induced by the map Grass $=\bigsqcup_{r} \operatorname{Grass}_{r}\left(k^{\infty}\right) \rightarrow \mathbb{N}$ sending $\operatorname{Grass}_{r}\left(k^{\infty}\right)$ to $r \in \mathbb{N}$, and thus is given by taking rank of vector bundles.

The group $K_{0}(X)$ is generated by classes of bundles generated by their global sections, so that by using the Whitney sum formula (which shows $x \mapsto \operatorname{rank}(x)+c\left(x^{*}\right)^{-1}$ is a homomorphism) and the fact that $S e g_{0}$ is a homomorphism, it suffices to verify $\operatorname{Seg}_{0}([E])=\operatorname{rank}(E)+c\left(E^{*}\right)^{-1}$ for such a bundle $E$. Moreover, the splitting principles for $K$-theory and motivic cohomology (which follow from the projective bundle formulas) and naturality allow us to assume $[E]$ is a sum of classes of line bundles each of which is generated by global sections.

Given a line bundle $L$ that is generated by its global sections, the value of $\operatorname{Seg}_{0, q}$ on $[L]$ (for $q \geq 1$ ) is given as follows: Choose a surjection $\mathcal{O}_{X}^{N+1} \rightarrow L$, which determines a map $X \rightarrow \mathcal{C}_{0}\left(\mathbb{P}^{\infty}\right)$ by taking the composition of

$$
X \rightarrow \mathbb{P}^{N} \hookrightarrow \mathbb{P}^{\infty} \hookrightarrow \mathcal{C}_{0}\left(\mathbb{P}^{\infty}\right) .
$$

The class of this map in $\pi_{0}\left|\operatorname{Hom}\left(X \times \Delta^{\bullet}, \mathcal{C}_{0} \mathbb{P}^{\infty}\right)_{1}^{+}\right|$gives $\sum_{q \geq 1} \operatorname{Seg}_{0, q}([L])$ under the isomorphism

$$
\pi_{0} \operatorname{Hom}\left(X \times \Delta^{\bullet}, \mathcal{C}_{0}\left(\mathbb{P}^{\infty}\right)\right)_{1}^{+} \cong \bigoplus_{q \geq 1} C H^{q}(X) .
$$

By naturality with respect to the morphism $X \rightarrow \mathbb{P}^{N}$, it suffices to take $X=\mathbb{P}^{N}, L=\mathcal{O}(1)$, and $\mathcal{O}_{X}^{N+1} \rightarrow L$ the canonical quotient. Observe $c\left(\mathcal{O}(1)^{*}\right)^{-1}=c(\mathcal{O}(-1))^{-1}=\left(1-H_{1}\right)^{-1}=1+H_{1}+H_{2}+\cdots \in\{1\} \times$ $\bigoplus_{q \geq 1} C H^{q}\left(\mathbb{P}^{N}\right)$, where $H_{i}$ denote the class of a hyperplane of codimension $i$ in $\mathbb{P}^{N}$. It therefore suffices to show that the class of the inclusion $\iota: \mathbb{P}^{N} \hookrightarrow \mathcal{C}_{0}\left(\mathbb{P}^{\infty}\right)$ corresponds to the element $H_{1}+H_{2}+\cdots \in$ $\bigoplus_{q \geq 1} C H^{q}\left(\mathbb{P}^{N}\right)$ under the isomorphism (1.5.1).

As argued in [FL1; 2.10], for each $q \geq 1$, the map

$$
\operatorname{Hom}\left(\mathbb{P}^{N} \times \Delta^{\bullet}, \mathcal{C}_{0}\left(\mathbb{P}^{\infty}\right)\right)_{1}^{+} \rightarrow \operatorname{Hom}\left(\mathbb{P}^{N} \times \Delta^{\bullet}, \mathcal{C}_{0}\left(\mathbb{P}^{q}\right)\right)^{+} / \operatorname{Hom}\left(\mathbb{P}^{N} \times \Delta^{\bullet}, \mathcal{C}_{0}\left(\mathbb{P}^{q-1}\right)\right)^{+}
$$


used to define the isomorphism (1.5.1) sends the map $\iota$ to the class of $\mathbb{P}^{N-q} \times \mathbb{P}^{q}$ regarded as a closed subscheme of $\mathbb{P}^{N} \times \mathbb{P}^{q}$ via $\left(\right.$ sym, $\left.\pi_{2}\right)$, where $s y m: \mathbb{P}^{N-q} \times \mathbb{P}^{q} \rightarrow \mathbb{P}^{N}$ is the "symmetrization map". We readily identify this class with the class of $H_{q} \subset \mathbb{P}^{N}$ in $C H^{q}\left(\mathbb{P}^{N}\right)$, as desired.

The final assertion holds since the classical total Chern class map is known to induce a rational isomorphism on smooth varieties.

We now proceed to develop our technique of "dimension shifting". We first introduce natural algebrogeometric models for products of spheres by adapting a construction of Thomason [Th; §3]. (Our approach differs from Thomason's slightly in that we use oriented simplicial complexes in order to more readily consider products.)

Let $M$ be a finite, oriented (abstract) simplicial complex with vertex set $V$. By this we mean that $V$ is a finite poset and that $M$ consists of a collection of totally ordered subsets of $V$ (these subsets are the "simplices" of $M$ ) which contains all singletons $\{v\}$ for $v \in V$ and is closed under taking subsets. A morphism $\theta: M \rightarrow N$ of oriented simplicial complexes is an order preserving function on vertex sets such that the image of a simplex of $M$ is a simplex of $N$. If $M$ and $N$ are two such oriented simplicial complexes with totally ordered vertex sets $V$ and $W$, then $M \times N$ is the oriented simplicial complex with vertex set $V \times W$ which is partially ordered by the rule $(v, w) \leq\left(v^{\prime}, w^{\prime}\right)$ if and only if $v \leq v^{\prime}$ and $w \leq w^{\prime}$. Moreover, a simplex of $M \times N$ is a subset $S$ of $V \times W$ which is totally ordered and has the property that the projections of $S$ to subsets of $V$ and $W$ are simplices of $M$ and $N$, respectively. For example, if $M$ and $N$ are $m$ - and $n$-simplices with totally ordered vertex sets $V=\left\{v_{0}<\cdots<v_{m}\right\}$ and $W=\left\{w_{0}<\cdots<w_{n}\right\}$ (so that every subset of $V$ is a simplex of $M$ and similarly for $N)$, then there are $\left(\begin{array}{c}n+m \\ b\end{array}\right)$ maximal simplices of $M \times N$ (each of dimension $m+n)$ having the form $\left\{\left(v_{i_{0}}, w_{j_{0}}\right),\left(v_{i_{1}}, w_{j_{1}}\right), \ldots,\left(v_{i_{m+n}}, w_{j_{m+n}}\right)\right\}$, where for all $s$, either $i_{s+1}=i_{s}$ and $j_{s+1}=j_{s}+1$ or $i_{s+1}=i_{s}+1$ and $j_{s+1}=j_{s}$.

Let $\mathcal{C}(M)$ denote the category whose objects are the simplices of $M$ and where a morphism is an inclusion of simplices (i.e., $\mathcal{C}(M)$ is the category associated to the poset of all simplices of $M$ ). For any simplex $\sigma=\left\{s_{0}, \ldots, s_{n}\right\}$ of $M$, define $\Delta_{\text {top }}^{\sigma}$ to be the subspace of $\mathbb{R} \cdot s_{0} \oplus \cdots \oplus \mathbb{R} \cdot s_{n} \cong \mathbb{R}^{n+1}$ consisting of points $\sum r_{i} s_{i}$ such that $r_{i} \geq 0$, for all $i$, and $\sum r_{i}=1$. Then $\sigma \mapsto \Delta_{\text {top }}^{\sigma}$ is a functor from $\mathcal{C}(M)$ to spaces. We define the geometric realization of $M,|M|$, to be the coequalizer of diagram of spaces

$$
\coprod_{\sigma_{0} \subset \sigma_{1} \in \operatorname{Arr}(\mathcal{C}(M))} \Delta_{\text {top }}^{\sigma_{1}} \rightrightarrows \coprod_{\sigma \in(\mathcal{C}(M))} \Delta_{\text {top }}^{\sigma} .
$$

Note that if $M$ and $N$ are two finite, oriented simplicial complexes, then $|M \times N|$ is naturally homeomorphic to $|M| \times|N|$.

In a parallel fashion, we associate to $M$ an affine variety which should be thought of as its "algebrogeometric realization" - namely, for any simplex $\sigma=\left\{s_{0}, \ldots, s_{n}\right\}$ of $M$, define $\Delta_{k}^{\sigma}$ to be the linear subvariety of the affine space $\mathbb{A}\left(k \cdot s_{0} \oplus \cdots \oplus k \cdot s_{n}\right) \cong \mathbb{A}^{n+1}$ defined by the equation $\sum x_{i}=1$, where $x_{i}$ is the $i$-th coordinate function relative to the basis $\left\{s_{0}, \ldots, s_{n}\right\}$. Then $\sigma \mapsto \Delta_{k}^{\sigma}$ is a functor from $\mathcal{C}(M)$ to $S c h / k$, and we define $|M|_{k}$, the algebro-geometric realization of $M$ over $k$, as the coequalizer in the category of $k$-schemes of the diagram

$$
\coprod_{\sigma_{0} \subset \sigma_{1} \in \operatorname{Arr}(\mathcal{C}(M))} \Delta_{k}^{\sigma_{1}} \rightrightarrows \coprod_{\sigma \in(\mathcal{C}(M))} \Delta_{k}^{\sigma}
$$

If the vertex set of $M$ is $\left\{v_{0}, \ldots, v_{m}\right\}$, then $|M|_{k}$ is the affine variety $\operatorname{Spec} k\left[x_{0}, \ldots, x_{m}\right] / I(M)$, where $I(M)$ is the ideal of generated by all monomials of the form $x_{i_{1}} \cdots x_{i_{t}}$ such that $v_{i_{1}}, \ldots, v_{i_{t}}$ does not span a simplex of $M$ (cf. [Th; 3.7], [DW]). As before, if $N$ is another finite, oriented simplicial complex, then $|M \times N|_{k}$ is naturally isomorphic to $|M|_{k} \times_{k}|N|_{k}$.

Define $T o p_{*}$ to be the category of pointed topological spaces and let $S m / k$ be the category of smooth, quasi-projective $k$-varieties. A contravariant functor $\mathcal{F}: S m / k \rightarrow T_{o p}$ is said to be homotopy invariant if the natural map $\mathcal{F}(X) \rightarrow \mathcal{F}\left(X \times \Delta_{k}^{n}\right)$ is a weak homotopy equivalence for all algebraic simplices $\Delta_{k}^{n}$. 
Lemma 1.6. Let $M$ be a finite, oriented simplicial complex and let $\mathcal{F}: S c h / k \rightarrow T_{\text {op }}$ be a contravariant functor that is homotopy invariant on smooth varieties.

(i) There is a natural chain of maps of spaces

$$
\mathcal{F}\left(|M|_{k}\right) \rightarrow \operatorname{holim}_{\mathcal{C}(M)} \mathcal{F} \stackrel{\sim}{\sim} \operatorname{holim}_{\mathcal{C}(M)} \mathcal{F}(\operatorname{Spec} k) \stackrel{\cong}{\longrightarrow} \operatorname{Maps}(|M|, \mathcal{F}(\operatorname{Spec} k)),
$$

whose second map is a weak equivalence and whose third map is a homeomorphism.

(ii) A morphism $X \times|M|_{k} \rightarrow Y$ with $X$ smooth determines a natural map

$$
\pi_{0} \mathcal{F}(Y) \rightarrow[|M|, \mathcal{F}(X)]
$$

(where $[-,-]$ denotes taking base-point free homotopy classes of maps).

(iii) If $\mathcal{F}$ takes values in group-like $H$-spaces, $M=M_{1} \times \cdots \times M_{m}$ with each $\left|M_{i}\right|$ homeomorphic to the sphere $S^{n_{i}}$, and $n=n_{1}+\cdots+n_{m}$, then a morphism $f: X \times|M|_{k} \rightarrow Y$ with $X$ smooth determines a natural map

$$
f^{\#}: \pi_{0} \mathcal{F}(Y) \rightarrow \pi_{n} \mathcal{F}(X) \text {. }
$$

Proof. In (1.6.1), the second map is a weak equivalence by homotopy invariance and [BK; XI.5.6], and the third is a homeomorphism by [BK; XI.4.2]. Thus, (ii) follows from contravariance of $\mathcal{F}$ together with (1.6.1) (for $\mathcal{F}$ replaced by $\mathcal{F}(X \times-)$ ). Finally, (iii) is proved by observing that the various projection maps $\pi_{i}:|M| \rightarrow\left|M_{1} \times \cdots \times \widehat{M}_{i} \times \cdots \times M_{m}\right|$ are split by the inclusions $\epsilon_{i}:\left|M_{1} \times \cdots \times \widehat{M}_{i} \times \cdots \times M_{m}\right| \cong$ $\left|M_{1} \times \cdots M_{i-1} \times p t \times M_{i+1} \times \cdots \times M_{m}\right| \rightarrow|M|$. Thus, $\pi_{n} \mathcal{F}(X)$ is the kernel of the split surjection

$$
[|M|, \mathcal{F}(X)] \rightarrow \prod_{i}\left[\left|M_{1} \times \cdots \times \widehat{M}_{i} \times \cdots \times M_{m}\right|, \mathcal{F}(X)\right] .
$$

Examples of functors $\mathcal{F}$ to which we apply the above considerations are: algebraic $K$-theory, $\mathcal{K}$; the functors $\mathcal{K}^{q}, q \geq 0$, used by Friedlander and Suslin to produce the motivic spectral sequence; the functors $\mathcal{H}_{\mathcal{M}}(-, \mathbb{Z}(q)), q \geq 0$, defined above; and the functor $\mathcal{H}_{\text {mult }}(-)$, also defined above. Thus, for $f: X \times|M|_{k} \rightarrow$ $Y$ as in Lemma 1.6 (iii), we have natural maps

$$
\begin{aligned}
& f^{\#}: K_{0}(Y) \rightarrow K_{n}(X) \\
& f^{\#}: K_{0}^{q}(Y) \rightarrow K_{n}^{q}(X), \quad q \geq 0 \\
& f^{\#}: H_{\mathcal{M}}^{2 q}(Y ; \mathbb{Z}(q)) \rightarrow H_{\mathcal{M}}^{2 q-n}(X ; \mathbb{Z}(q)), \quad q \geq 0 \\
& f^{\#}: \pi_{0} \mathcal{H}_{\text {mult }}(Y) \rightarrow \pi_{n} \mathcal{H}_{\text {mult }}(X) .
\end{aligned}
$$

In the special case of algebraic $K$-theory, we have the following two key additional properties (Propositions 1.7 and 1.8).

Proposition 1.7. Let $M$ be a finite, oriented simplicial complex and let $X$ be an affine, smooth k-variety. Then the natural augmentation map

$$
\mathcal{K}\left(X \times|M|_{k}\right) \rightarrow \underset{\mathcal{C}(M)}{\operatorname{holim}} \mathcal{K}(X \times-)
$$

induces an isomorphism on $\pi_{n}$ for $n=0,1$. Consequently, there is an isomorphism

$$
K_{0}\left(X \times|M|_{k}\right) \cong[|M|, \mathcal{K}(X)]
$$


which is natural in both $X$ and $M$.

Proof. Consider the natural transformation of functors $\mathcal{K} \rightarrow \mathcal{K} H$, where $\mathcal{K} H(X)$ is Weibel's homotopy $K$-theory [We]. Recall that $\mathcal{K}(U) \rightarrow \mathcal{K} H(U)$ is a weak equivalence for $U$ smooth [We; 6.10]. Moreover, $\mathcal{K} H(X \times-)$ satisfies the property that for any decomposition of a finite simplicial complex as a union of two subcomplexes $-M=M_{1} \cup M_{2}$ - the square

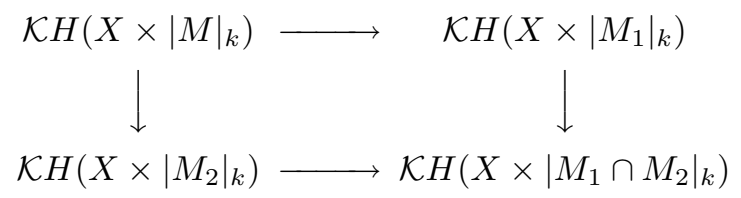

is homotopy Cartesian [We; 4.10]. Consequently, we have by [Th; 3.15] that

$$
\mathcal{K} H\left(X \times|M|_{k}\right) \rightarrow \underset{\mathcal{C}(M)}{\operatorname{holim}} \mathcal{K} H(X \times-)
$$

is a weak homotopy equivalence. Hence, to prove that $\mathcal{K}\left(X \times|M|_{k}\right) \rightarrow \operatorname{holim}_{\mathcal{C}(M)} \mathcal{K}(X \times-)$ induces an isomorphism on $\pi_{n}$ for $n=0,1$, it suffices to prove $\mathcal{K}\left(X \times|M|_{k}\right) \rightarrow \mathcal{K} H\left(X \times|M|_{k}\right)$ induces an isomorphism on $\pi_{n}$ for $n=0,1$. This follows from [We; 1.5], using the fact that $X \times|M|_{k}$ is $K_{1}$-regular by [DW].

The isomorphism $K_{0}\left(X \times|M|_{k}\right) \cong[|M|, \mathcal{K}(X)]$ follows from Lemma 1.6(i).

In order to employ a dimension shifting argument in conjunction with Adams operations, we require the following compatibility result.

Proposition 1.8. Let $M=M_{1} \times \cdots \times M_{m}$ be a finite, oriented simplicial complex with $\left|M_{i}\right| \cong S^{n_{i}}$, for all $i$, and $n_{1}+\cdots+n_{m}=n$, and let $f: X \times|M|_{k} \rightarrow Y$ be a morphism, where $Y$ is a smooth quasi-projective $k$-variety and $X$ is a smooth, affine $k$-variety. Then the map

$$
f^{\#}: K_{0}(Y) \rightarrow K_{n}(X)
$$

defined above commutes with the Adams operations $\psi^{k}, k \geq 0$.

Moreover, for $X$ a smooth, affine k-variety, given an element $\gamma$ of $K_{n}(X)$ (respectively, $K_{n}(X)_{\mathbb{Q}}^{(q)}$ for some $q \geq 0$ ), there is a smooth $k$-variety $Y$ and a morphism $f: X \times|M|_{k} \rightarrow Y$ such that $\gamma$ lies in the image of $f^{\#}: K_{0}(Y) \rightarrow K_{n}(X)$ (respectively, $\left.f^{\#}: K_{0}(Y)_{\mathbb{Q}}^{(q)} \rightarrow K_{n}(X)_{\mathbb{Q}}^{(q)}\right)$.

Proof. In [Gr] D. Grayson constructs Adams operations $\Psi^{k}$, for $k>0$, on the level of $K$-theory spaces, by defining natural transformations of functors from $S c h / k$ to the category of pointed topological spaces which sends $X$ to

$$
\Psi^{k}:|S \bullet \mathcal{P}(X)| \rightarrow\left|S \bullet \tilde{G}^{(k)} \mathcal{P}(X)\right|
$$

Here, $S_{\bullet}$ is the $S$-construction of Waldhausen, $\mathcal{P}(X)$ is (a suitably functorial model for) the exact category of coherent, locally free sheaves on $X$, and $\tilde{G}^{(k)}$ arises from a $(k-1)$ dimensional cube of exact categories. In light of the natural weak equivalences $\mathcal{K}(X) \sim \Omega|S \bullet \mathcal{P}(X)|$ and $\mathcal{K}(X) \sim \Omega\left|S_{\bullet} \tilde{G}^{(k)} \mathcal{P}(X)\right|$, these maps induce operations $\Psi^{k}$ on the $K$-groups of $X$, which we refer to as "Grayson's Adams operations". 
Since the maps of spaces $\Psi^{k}$ are strictly functorial, we obtain the commutative diagram

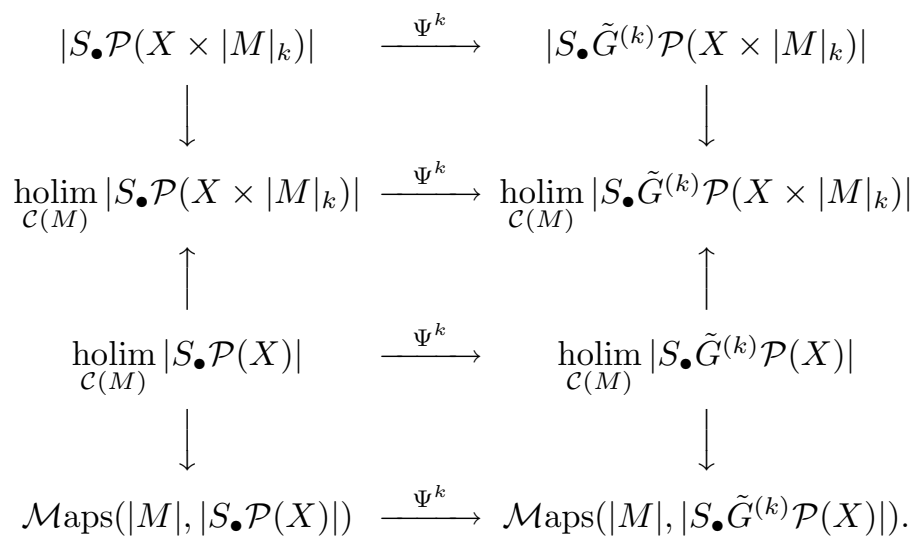

In particular, the isomorphism

$$
K_{0}\left(X \times|M|_{k}\right) \cong[M, \mathcal{K}(X)]
$$

of Proposition 1.7 commutes with Grayson's Adams operations, and so does the projection map $K_{0}(X \times$ $\left.|M|_{k}\right) \rightarrow K_{n}(X)$. Since $X$ and $Y$ are regular, the Grayson's Adams operations are known to agree with the classical ones (cf. discussion in [Le; §2]).

To prove the second assertion, recall from $[\mathrm{BFM}]$ that for any (possibly singular) variety $V$, we have

$$
K_{0}(V) \cong \varliminf_{V \rightarrow U} K_{0}(U)
$$

where the (non-filtered) limit ranges over morphisms $V \rightarrow U$ with $U$ smooth. Since the the natural transformation of functors on $S c h / k$ given by $K_{0}(U) \mapsto K_{0}(U)_{\mathbb{Q}}^{(q)}$ is exact, we have $K_{0}(V)_{\mathbb{Q}}^{(q)}=\varliminf_{V \rightarrow U} K_{0}(U)_{\mathbb{Q}}^{(q)}$. The result follows by taking $V=X \times|M|_{k}$ in (1.8.1), using the isomorphism of 1.7, and using that the map $K_{0}\left(X \times|M|_{k}\right) \rightarrow K_{n}(X)$ commutes with Grayson's Adams operations.

Following the construction of Friedlander and Suslin [FS; $\S 8$ ], for any $U \in S c h / k$ define $\mathcal{K}^{Q, \mathbb{A}^{q}}(U)$ to be the simplicial set giving the $K$-theory (as defined in [TT]) of the Waldhausen category of perfect complexes on $U \times \mathbb{A}^{q}$ supported on subvarieties which are quasi-finite over $U$. Note that using the technique of [FS; App. C] we may view $\mathcal{K}^{Q, \mathbb{A}^{q}}(-)$ as a (strict) functor from $S c h / k$ to simplicial sets. As in [FS; D.6], there is a natural transformation $\mathcal{K}^{Q, \mathbb{A}^{q}} \rightarrow \mathcal{K}^{q}$ of functors from $S c h / k$ to simplicial sets such that $\mathcal{K}^{q}$ is flasque (for the Zariski topology) and $\mathcal{K}^{Q, \mathbb{A}^{q}} \rightarrow \mathcal{K}^{q}$ is locally a weak equivalence. Loosely speaking, $\mathcal{K}^{q}$ is the functor from $S c h / k$ to simplicial sets given by imposing Zariski descent on $\mathcal{K}^{Q, \mathbb{A}^{q}}$. (In [FS], the functors $\mathcal{K}^{Q, \mathbb{A}^{q}}$ and $\mathcal{K}^{q}$ are defined only on $S m / k$ and, moreover, the functor $\mathcal{K}^{q}$ is derived from $\mathcal{K}^{Q, \mathbb{A}^{q}}$ by first fixing an $X$ and the looking only at the small Zariski site of $X$. However, the constructions carry over to this more general context without any difficulties: to define $\mathcal{K}^{q}$ from $\mathcal{K}^{Q, \mathbb{A}^{q}}$ on all of $S c h / k$, one forms the Godement resolution on the big Zariski site $(S c h / k)_{Z a r}$ of the Kan extension of the functor $\mathcal{K}^{Q, \mathbb{A}^{q}}$.)

We also write $\mathcal{K}^{q}$ for the associated functor from $S c h / k$ to spaces obtained by taking geometric realizations. Then there is an infinite tower of spaces

$$
\cdots \rightarrow \mathcal{K}^{q+1}(X) \rightarrow \mathcal{K}^{q}(X) \rightarrow \cdots \rightarrow \mathcal{K}^{0}(X)
$$

which is natural with respect to $X \in S c h / k$ and satisfies $\mathcal{K}^{0}(X) \sim \mathcal{K}(X)$ for $X$ smooth. Moreover, for smooth varieties each step in the tower fits in a homotopy fibration sequence of the form

$$
\mathcal{K}^{q+1}(X) \rightarrow \mathcal{K}^{q}(X) \rightarrow \mathcal{H}_{\mathcal{M}}(X, \mathbb{Z}(q)),
$$


natural with respect to $X \in S m / k$ [FS; 13.6]. Moreover, by [Le; 9.7] the associated spectral sequence admits natural operations $\Phi_{k}, k \geq 1$, such that the action of $\Phi_{k}$ on $\mathcal{H}_{\mathcal{M}}(X, \mathbb{Z}(q))$ is multiplication by $k^{q}$ and the operations on the homotopy groups of $\mathcal{K}^{0}(X) \sim \mathcal{K}(X)$ are the usual Adams operations. Using the convergence of the spectral sequence, this shows the sequences (1.9.1) split on the the level of rational homotopy groups, giving short exact sequences of the form

$$
0 \rightarrow K_{n}^{q+1}(X)_{\mathbb{Q}} \rightarrow K_{n}^{q}(X)_{\mathbb{Q}} \rightarrow H_{\mathcal{M}}^{2 q-n}(X, \mathbb{Q}(q)) \rightarrow 0
$$

for all $q \geq 0$ and $X \in S m / k$, and that the induced filtration on $K_{n}(X)_{\mathbb{Q}}$,

$$
\ldots \hookrightarrow K_{n}^{q+1}(X)_{\mathbb{Q}} \hookrightarrow K_{n}^{q}(X)_{\mathbb{Q}} \hookrightarrow \ldots \hookrightarrow K_{n}^{0}(X)_{\mathbb{Q}}=K_{n}(X)_{\mathbb{Q}},
$$

is the usual gamma filtration. In particular, the inclusion $K_{n}(X)_{\mathbb{Q}}^{(q)} \hookrightarrow K_{n}(X)_{\mathbb{Q}}$ factors through $K_{n}^{q}(X)_{\mathbb{Q}} \hookrightarrow$ $K_{n}(X)_{\mathbb{Q}}$ and the induced map

$$
\theta_{n, q}: K_{n}(X)_{\mathbb{Q}}^{(q)} \hookrightarrow K_{n}^{q}(X)_{\mathbb{Q}} \rightarrow H_{\mathcal{M}}^{2 q-n}(X, \mathbb{Q}(q)),
$$

is a natural isomorphism of contravariant functors from $S m / k$ to $\mathbb{Q}$ vector spaces.

The maps $f^{\#}$ on $K$-theory given in Proposition 1.8 satisfy the following compatibilities with the similarly defined $f^{\#}$ on motivic cohomology.

Proposition 1.9. Let $M=M_{1} \times \cdots \times M_{m}$ be a finite, oriented simplicial complex with $\left|M_{i}\right| \cong S^{n_{i}}$, for all $i$, and $n_{1}+\cdots+n_{m}=n$, and let $f: X \times|M|_{k} \rightarrow Y$ be a morphism with $X$ and $Y$ both smooth. Then for any $q \geq 0$, the following diagrams commute:

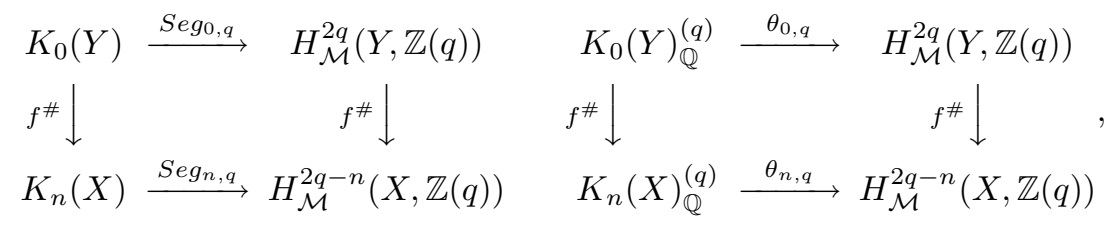

where $\theta_{n, q}$ is the isomorphism of (1.9.2).

Proof. The commutativity of the first diagram arises from the naturality of the construction of Lemma 1.6 (iii) applied to the natural transformation $S e g: \mathcal{K}(X) \rightarrow \mathcal{H}_{\text {mult }}(X)$ and the observation that the map $f^{\#}: \pi_{0} \mathcal{H}_{\text {mult }}(Y) \rightarrow \pi_{n} \mathcal{H}_{\text {mult }}(X)$ respects the natural splitting on the homotopy groups of $\mathcal{H}_{\text {mult }}(X)$ and $\mathcal{H}_{\text {mult }}(Y)$.

The second diagram is seen to commute by using Lemma 1.6 (iii) once again and noting that the maps $\theta_{i, q}$ are induced by natural transformations of space-valued functors $\mathcal{K}^{q}(-) \rightarrow \mathcal{H}_{\mathcal{M}}(-, \mathbb{Z}(q))$.

We now can apply our dimension-shifting technique to prove that $S e g$ is a rational equivalence, thereby extending Proposition 1.5.

Theorem 1.10. For any smooth variety $X$, the natural transformation

$$
\text { Seg }: \mathcal{K}(X) \rightarrow \mathcal{H}_{\text {mult }}(X)
$$

is a rational homotopy equivalence.

Proof. Let $p: X^{\prime} \rightarrow X$ denote an affine vector bundle torsor - that is, $X^{\prime}$ is affine and locally for the Zariski topology on $X$, the map $p$ is given by a map of the form $\pi_{1}: U \times \mathbb{A}^{n} \rightarrow U$ (cf. $[\mathrm{J}]$ ). Since the maps 
$p^{*}: \mathcal{K}(X) \rightarrow \mathcal{K}\left(X^{\prime}\right)$ and $p^{*}: \mathcal{H}_{\text {mult }}(X) \rightarrow \mathcal{H}_{\text {mult }}\left(X^{\prime}\right)$ are weak equivalences and $S e g$ is natural, we may assume without loss of generality that $X$ is affine.

It suffices to prove that each

$$
S e g_{n} \otimes \mathbb{Q}: K_{n}(X)_{\mathbb{Q}} \rightarrow H_{\mathcal{M}}^{-n}(X, \mathbb{Q}(0)) \times\left(\{1\} \times \oplus_{q>0} H_{\mathcal{M}}^{2 q-n}(X, \mathbb{Q}(q))^{\times}\right.
$$

is an isomorphism. For $n \geq 0$, give $K_{n}(X)_{\mathbb{Q}}$ the gamma filtration and filter $\pi_{n} \mathcal{H}_{\text {mult }}(X)$ by the index $q$. For $n=0$, the map $S e g_{0}$ coincides with the classical total Segre map on $K_{0}(X)$ up to a filtration preserving automorphism of $K_{0}$ by Proposition 1.5, and thus induces a filtration preserving rational isomorphism by the Grothendieck Riemann-Roch Theorem [SGA6], [BS] (see also [Fu; 15.3.6]).

For any smooth variety $Y$, elements of $\pi_{0} \mathcal{K}^{q}(Y)$ are represented by coherent sheaves on $Y$ supported on subschemes of codimension $q$ and the map $\pi_{0} \mathcal{K}^{q}(Y) \rightarrow H_{\mathcal{M}}^{2 q}(Y, \mathbb{Z}(q)) \cong C H^{q}(Y)$ is given by sending such a sheaf to the codimension $q$ cycle of its support [FS; 8.6]. In particular, the map $\theta_{0, q}: K_{n}(Y)_{\mathbb{Q}}^{(q)} \rightarrow$ $H_{\mathcal{M}}^{2 q}(Y, \mathbb{Q}(q)) \cong C H^{2 q}(Y)_{\mathbb{Q}}$ sends $\left[\mathcal{O}_{Z}\right]$ to $[Z]$, for any codimension $q$ integral subscheme $Z$ of $Y$. Using Grothendieck's Riemann-Roch Theorem [SGA6], [BS], one sees that the $q$-th component of the Chern character map $c h_{0, q}: K_{0}(Y)_{\mathbb{Q}} \rightarrow C H^{q}(Y)_{\mathbb{Q}}$ also sends such a class $\left[\mathcal{O}_{Z}\right]$ to $[Z]$ (cf. [Fu; 15.2.16]), so that $\theta_{0, q}$ and $c h_{0, q}$ coincide on $K_{0}(Y)_{\mathbb{Q}}^{(q)}$.

Given $f: X \times|M|_{k} \rightarrow Y$ with $\left|M_{i}\right| \cong S^{n_{i}}$, for all $i$, and $n_{1}+\cdots+n_{m}=n$ the map $f^{\#}: K_{0}(Y) \rightarrow K_{n}(X)$ given by Lemma 1.6 (iii) commutes with the Adams operations by Proposition 1.8, and hence $f^{\#}$ is filtration preserving. Using Proposition 1.8 again and the first square of Proposition 1.9, we have that $S e g_{n}$ is filtration preserving. It thus suffices to show that the map induced by $S e g_{n}$ on associated graded objects,

$$
\bigoplus_{q} \operatorname{Seg}_{n, q}: \bigoplus_{q} K_{n}(X)_{\mathbb{Q}}^{(q)} \rightarrow \bigoplus_{q} H_{\mathcal{M}}^{2 q-n}(X, \mathbb{Q}(q))
$$

is an isomorphism. For $\alpha \in K_{0}(Y)_{\mathbb{Q}}^{(q)}$, we have

$$
\theta_{0, q}(\alpha)=\operatorname{ch}_{0, q}(\alpha)=\frac{c_{q}(\alpha)}{(q-1) !}=-\frac{\operatorname{Seg}_{0, q}\left(\alpha^{*}\right)}{(q-1) !}
$$

by the result proven above and Proposition 1.5. Thus, the commutative squares of Proposition 1.9 imply that

$$
\operatorname{Seg}_{n, q}(x)=-(q-1) ! \theta_{n, q}\left(x^{*}\right)
$$

for all $x \in K_{n}(X)^{(q)}$ belonging to the image of $f^{\#}$, for all $f$ as above. Using that $\theta_{n, q}$ is an isomorphism together with Proposition 1.8, we see that $S e g_{n, q}$ is an isomorphism as required.

In the following remark, we describe the effect of $S e g_{n}$ on a set of generators of $K_{n}(X)$ in terms of universal classes on Grassmannians.

Remark 1.11. Let $U_{r}^{N}$ denote the universal rank $r$ bundle on $\operatorname{Grass}_{r}\left(k^{N}\right)$. For any smooth variety, the set

$$
\left.\left\{\left.f^{\#}\left(U_{r}^{N}\right) \quad|\quad f: X \times| M\right|_{k} \rightarrow \operatorname{Grass}_{r}\left(k^{N}\right)\right), 0<r<N\right\}
$$

with $|M| \cong S^{n}$, generates generates $K_{n}(X)$ as an abelian group. The components of the total Segre map, given as the composition

$$
s_{n, q}: K_{n}(X) \stackrel{(-)^{*}}{\longrightarrow} K_{n}(X) \stackrel{\operatorname{Seg}_{n}}{\longrightarrow} H_{\mathcal{M}}^{-n}(X, \mathbb{Z}(0)) \oplus\left(\{1\} \times \bigoplus_{q \geq 0} H_{\mathcal{M}}^{2 q-n}(X, \mathbb{Z}(q))\right)^{\times} \rightarrow H_{\mathcal{M}}^{2 q-n}(X, \mathbb{Z}(q)),
$$


send $f^{\#}\left(U_{r}^{N}\right)$ to

$$
f^{\#}\left(s_{0, q}\left(U_{r}^{N}\right)\right) \in H_{\mathcal{M}}^{2 q}\left(\operatorname{Grass}_{r}\left(k^{N}\right), \mathbb{Z}(q)\right) .
$$

Consequently, the universal elements $U_{r}^{N}$ and $s_{0, q}\left(U_{r}^{N}\right)$ completely determine the behavior of the Segre class maps $s_{n . q}: K_{n}(X) \rightarrow H_{\mathcal{M}}^{2 q-n}(X, \mathbb{Z}(q))$.

To complete our discussion of the rational isomorphism relating algebraic $K$-theory and motivic cohomology, we consider the Chern character ch related to the our Segre map Seg and prove that it is an isomorphism of graded algebras.

Recall that the classical Chern character map $c_{0}: K_{0}(X) \rightarrow C H^{*}(X)_{\mathbb{Q}} \cong \bigoplus_{q} H_{\mathcal{M}}^{2 q}(X, \mathbb{Q}(q))$ is defined from the individual Chern class maps (and, consequently, from the individual Segre class maps) via universal polynomials. That is, the $q$-th component of $c h_{0}$

$$
c h_{0, q}: K_{0}(X) \rightarrow H_{\mathcal{M}}^{2 q}(X, \mathbb{Q}(q)), \quad q \geq 1,
$$

is defined by $c h_{0, q}=P_{q}\left(s_{0,0}, \ldots, s_{0, q}\right)$, where $P_{q}\left(x_{0}, \ldots, x_{q}\right)$ is a certain polynomial with rational coefficients having degree $q$, where the degree of $x_{i}$ is defined to be $i$. (The map $c h_{0,0}$ is by definition the rank map $K_{0}(X) \rightarrow H_{\mathcal{M}}^{0}(X, \mathbb{Q})$.) We define the higher Chern character map

$$
c h_{n, q}: K_{n}(X) \rightarrow H_{\mathcal{M}}^{2 q-n}(X, \mathbb{Q}(q))
$$

via the formula $c h_{n, q}(\gamma)=P_{q}\left(s_{n, 0}(\gamma), \ldots, s_{n, q}(\gamma)\right)$, using the same polynomial $P_{q}\left(x_{1}, \ldots, x_{q}\right)$. If $n>0$, every product of the form $s_{n, i} s_{n, j}$ with $i, j \geq 1$ is defined to be zero, so that this polynomial expression simplifies to be

$$
c h_{n, q}=(-1)^{q} \frac{1}{(q-1) !} s_{n, q}, \quad n \geq 1 .
$$

The following theorem establishes that $c h$ is a graded ring isomorphism. In the sense that Theorem 1.12 incorporates the ring structure, it presents a stronger result than Theorem 1.10; however, unlike Seg, the map $c h$ is defined only on rational homotopy groups.

Theorem 1.12. For any smooth variety $X$, the Chern character map

$$
c h: \bigoplus_{n} K_{n}(X) \rightarrow \bigoplus_{n, q} H_{\mathcal{M}}^{2 q-n}(X, \mathbb{Q}(q))
$$

is a graded ring homomorphism which induces a rational isomorphism

$$
c h: \bigoplus_{n} K_{n}(X)_{\mathbb{Q}} \cong \bigoplus_{n, q} H_{\mathcal{M}}^{2 q-n}(X, \mathbb{Q}(q)) .
$$

Remark. Observe that $H_{\mathcal{M}}^{2 q-n}(X, \mathbb{Z}(q)) \cong C H^{q}(X, n)=0$ for $q>n+\operatorname{dim}(X)$. In particular, for a given $n$, the group $K_{n}(X)_{\mathbb{Q}}$ is isomorphic under the Chern character to a finite sum of cohomology groups.

Proof of 1.12. As in the proof of Theorem 1.10, we may replace $X$ with an affine vector bundle torsor, so that without loss of generality, we may assume $X$ is affine.

We use that the product structures on $K$-theory and motivic cohomology are given by external pairings

$$
\mathcal{K}(X) \wedge \mathcal{K}(Y) \rightarrow \mathcal{K}(X \times Y)
$$

and

$$
\mathcal{H}_{\mathcal{M}}(X, \mathbb{Z}(p)) \wedge \mathcal{H}_{\mathcal{M}}(Y, \mathbb{Z}(q)) \rightarrow \mathcal{H}_{\mathcal{M}}(X \times Y, \mathbb{Z}(p+q))
$$


which are natural up to homotopy. Let $M$ and $N$ be finite, oriented simplicial complexes homeomorphic to the spheres $S^{m}$ and $S^{n}$ respectively, and assume morphisms $f: X \times|M|_{k} \rightarrow U$ and $g: X \times|N|_{k} \rightarrow V$ are given with $U$ and $V$ smooth. The diagram

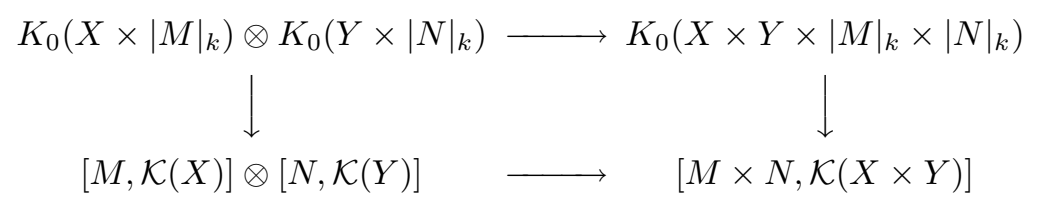

commutes by naturality of the pairing $\mathcal{K}(X) \times \mathcal{K}(Y) \rightarrow \mathcal{K}(X \times Y)$. Since this pairing factors through $\mathcal{K}(X) \wedge \mathcal{K}(Y)$, one deduces the commutativity of

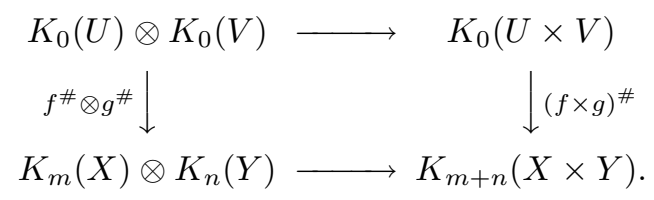

Similarly, the square

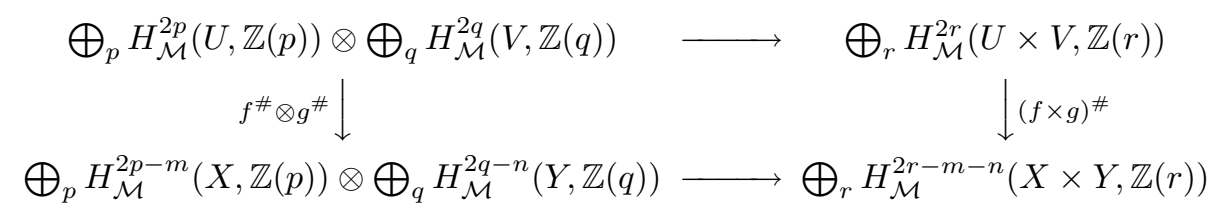

commutes. Consider the cubical diagram formed by mapping (1.12.1) to (1.12.2) using $c h_{0} \otimes c h_{0}, c h_{0}$, $c h_{m} \otimes c h_{n}$, and $c h_{m+n}$. Using the fact that the classical Chern character map $c h_{0}$ is multiplicative [BS] and the commutativity of the first square of Proposition 1.9, we see that each face of this cube commutes except possibly the face

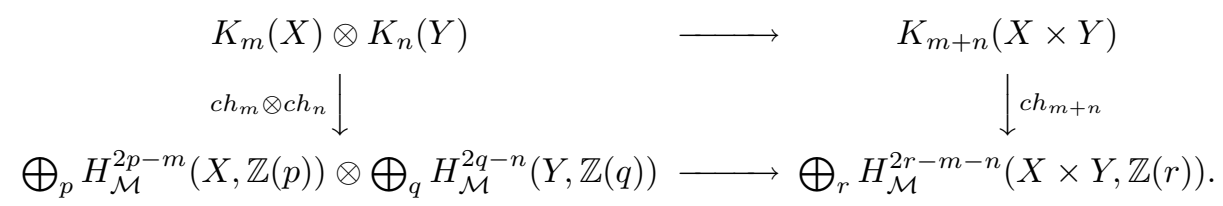

Hence (1.12.3) commutes upon restriction to the image of $K_{0}(U) \otimes K_{0}(V) \rightarrow K_{m}(X) \otimes K_{n}(Y)$. As $U, V$, $f$, and $g$ range over all possibilities, the union of such images forms a generating set of $K_{m}(X) \otimes K_{n}(Y)$ by Proposition 1.8. Thus, we conclude that (1.12.3) commutes. By taking $X=Y$ and using naturality of the Chern character with respect to the pullback along the diagonal map $X \hookrightarrow X \times X$, the multiplicativity of the Chern character follows.

As recalled previously, the map $c h_{0}$ is a rational isomorphism (cf. [Fu; 15.2.16]). For $n>0$, we have

$$
c h_{n, q}=(-1)^{q} \frac{1}{(q-1) !} s_{n, q}=(-1)^{q} \frac{1}{(q-1) !} \operatorname{Seg}_{n, q} \circ(-)^{*}
$$

so that Theorem 1.10 implies that $c h$ is an isomorphism. 


\section{$\S 2$ Semi-topological Singular COMplex}

As we discussed in the introduction, our purpose in this section is to prove a recognition principle (Theorem 2.3 ) which will enable us to conclude that certain natural transformations $F \rightarrow G$ of functors on $S c h / \mathbb{C}$, the category of quasi-projective complex varieties, with values in topological spaces induce weak homotopy equivalences of the form $\left|F\left(\Delta_{\text {top }}^{\bullet}\right)\right| \rightarrow\left|G\left(\Delta_{\text {top }}^{\bullet}\right)\right|$.

We begin with the functor

$$
(-)^{a n}: S c h / \mathbb{C} \rightarrow T o p
$$

sending a variety $X$ to its analytic realization $X^{a n}$ and a morphism $f: X \rightarrow Y$ of varieties to the continuous map $f^{a n}$ it determines. Here, $S c h / \mathbb{C}$ is the category of quasi-projective complex varieties and Top denotes the category of Hausdorff spaces and continuous maps. Given a contravariant functor $F$ defined on $S c h / \mathbb{C}$ taking values in a category $\mathcal{C}$ closed under colimits (e.g., sets, simplicial sets, spaces, spectra), we "promote" $F$ to take values from Top using the usual Kan extension formula. That is, given $T \in T o p$, define $\operatorname{Var}^{T}$ to be the category where objects are continuous maps $T \rightarrow U^{a n}$, for $U \in S c h / \mathbb{C}$, and a morphism from $T \rightarrow U^{a n}$ to $T \rightarrow V^{a n}$ is a morphism of varieties $U \rightarrow V$ causing the evident triangle to commute. Define the value of $F$ on a topological space $T$ to be

$$
F(T)=\varliminf_{\left(T \rightarrow U^{a n}\right)} F(U),
$$

where the colimit is indexed by $\left(\operatorname{Var}^{T}\right)^{o p}$.

In particular, given such an $F$ and letting $\mathcal{C}$ be the category of sets, simplicial sets, spaces, or spectra, we define $F\left(\Delta_{\text {top }}^{\bullet}\right)$ to be the simplicial object in $\mathcal{C}$ given by $n \mapsto F\left(\Delta_{\text {top }}^{n}\right)$ and we define $\left|F\left(\Delta_{\text {top }}^{\bullet}\right)\right|$ to be the geometric realization of this simplicial object:

$$
\left|F\left(\Delta_{t o p}^{\bullet}\right)\right|=\left|n \mapsto F\left(\Delta_{t o p}^{n}\right)\right| .
$$

As alluded to in the introduction, a main difficulty in understanding the homotopy type of $\left|\mathcal{F}\left(\Delta_{\text {top }}\right)\right|$ (for example, when $\mathcal{F}=\mathcal{K}(X \times-))$ is that the indexing category $\operatorname{Var}^{\Delta_{\text {top }}^{n}}$ involves maps $\Delta_{\text {top }}^{n} \rightarrow U^{a n}$ with $U$ singular. Theorem 2.3 and its consequences allow us to overcome this difficulty - in particular, Corollary 2.7 below shows that if a natural transformation $\phi: F \rightarrow G$ of group like $\mathrm{H}$-spaces is a weak equivalence when restricted to smooth varieties, then $\phi$ induces a weak equivalence $\left|F\left(\Delta_{\text {top }}^{\bullet}\right)\right| \stackrel{\sim}{\longrightarrow}\left|G\left(\Delta_{\text {top }}^{\bullet}\right)\right|$.

In order to prove our recognition principle (Theorem 2.3), we first formulate an appropriate Grothendieck topology. The following topology is essentially due to P. Deligne.

Definition 2.1. (cf. $[\mathrm{D}, \S 5]$ ) We say that a continuous map $f: S \rightarrow T$ of topological spaces is a map of cohomological descent if for every sheaf of abelian groups $F$ defined on $T$ the natural map

$$
H^{*}(T, F) \rightarrow H^{*}\left(N_{T}(S), f^{*} F\right)
$$

is an isomorphism, where $N_{T}(S)$ is the simplicial space given at level $n$ by the $n$-fold fibre product of $S$ over $T$ and the natural map is that induced by the augmentation $N_{T}(S) \rightarrow T$. Such a map $f: S \rightarrow T$ is said to be a map of universal cohomological descent if the pull-back $f^{\prime}: S^{\prime}=S \times_{T} T^{\prime} \rightarrow T^{\prime}$ is a map of cohomological descent for every continuous map $T^{\prime} \rightarrow T$.

The topology of universal analytic descent, $(S c h / \mathbb{C})_{\text {uad }}$, is the Grothendieck topology associated to the pre-topology on $S$ ch $/ \mathbb{C}$ whose coverings are finite collections of morphisms $\left\{f_{i}: X_{i} \rightarrow Y\right\}$ such that the induced map $f: \coprod_{i} X_{i}^{a n} \rightarrow Y^{a n}$ is a map of universal cohomological descent.

By Hironaka's resolution of singularities [H] (or by de Jong's proof of the existence of smooth modifications for singular varieties [DeJ]), if $Y$ is a singular variety then there exists a proper surjective map $X \rightarrow Y$ with 
$X$ smooth. As we recall below, such a map is a uad-covering - a key observation which will enable us to understand certain properties of presheaves on $S c h / \mathbb{C}$ by restricting attention to smooth varieties.

We recall Voevodsky's $h$ topology [V1], which is defined in terms of the Grothendieck pre-topology on $S c h / \mathbb{C}$. Namely, a covering of $U \in S c h / \mathbb{C}$ is a finite collection of morphisms $\left\{U_{i} \rightarrow U\right\}$ such that $\coprod_{i} U_{i} \rightarrow U$ is a universal quotient map - that is, given any morphism $V \rightarrow U$, the underlying map of Zariski spaces associated to $\coprod_{i} U_{i} \times_{U} V \rightarrow V$ is a quotient map. The $h$ topology is strictly finer than the étale topology.

Lemma 2.2. The uad topology is finer than the $h$ topology. In particular, if $\phi: F \rightarrow G$ is a map of presheaves on $S c h / \mathbb{C}$ such that the induced map of associated $h$ sheaves $\phi_{h}$ is an isomorphism, then the map of associated uad sheaves $\phi_{\text {uad }}: F_{\text {uad }} \rightarrow G_{\text {uad }}$ is also an isomorphism.

Proof. It suffices to show that every $h$-covering admits a refinement by a uad-covering. By [FV; 10.4], every $h$-covering $\left\{Y_{i} \rightarrow X\right\}$ admits a refinement $\left\{V_{j} \rightarrow X\right\}$ such that each $V_{j} \rightarrow X$ factors as $V_{j} \rightarrow X^{\prime} \rightarrow X$, where $X^{\prime} \rightarrow X$ is a surjective proper map and the $V_{j}$ 's form a Zariski open cover of $X^{\prime}$. A Zariski open covering is a uad-covering; moreover, $f^{a n}:\left(X^{\prime}\right)^{a n} \rightarrow X^{a n}$ is a morphism of universal cohomological descent whenever $X^{\prime} \rightarrow X$ is proper and surjective by [SGA4; Vbis4.1.1]. The result follows.

The main result of this section is the following theorem, inspired by a result of Voevodsky [V2; 5.9].

Theorem 2.3. Let $F \rightarrow G$ be a morphism of presheaves of abelian groups on $S c h / \mathbb{C}$ such that $F_{\text {uad }} \rightarrow G_{\text {uad }}$ is an isomorphism. Then the natural map of simplicial abelian groups

$$
F\left(\Delta_{\text {top }}^{\bullet}\right) \rightarrow G\left(\Delta_{\text {top }}^{\bullet}\right)
$$

is a homotopy equivalence.

Proof. For any abelian group $A$, let $A^{\#}$ denote the dual abelian group $\operatorname{Hom}_{\mathbb{Z}}(A, \mathbb{Q} / \mathbb{Z})$. For $d \geq 0$, let $Z^{d}$ denote the presheaf on $S c h / \mathbb{C}$ defined by

$$
Z^{d}(U)=\left(\mathbb{Z} \operatorname{Hom}_{\text {spaces }}\left(\Delta_{\text {top }}^{d}, U^{a n}\right)\right)^{\#},
$$

the dual of the singular $d$-chains of $U^{a n}$. The collection of presheaves $Z^{d}, d \geq 0$, forms a cosimplicial presheaf of abelian groups in the evident manner, and we let $Z \bullet$ denote the associated bounded below cochain complex of presheaves. Note that the cohomology of the cochain complex $Z \bullet(U)$ gives the singular cohomology with $\mathbb{Q} / \mathbb{Z}$ coefficients of the space $U^{a n}$ :

$$
h^{n} Z \bullet(U)=H_{\text {sing }}^{n}\left(U^{a n}, \mathbb{Q} / \mathbb{Z}\right) .
$$

We first verify for any abelian presheaf $F$ on $S c h / \mathbb{C}$ the isomorphism of abelian groups

$$
\operatorname{Hom}_{p s h v s}\left(F, Z^{d}\right) \cong F\left(\Delta_{\text {top }}^{d}\right)^{\#} .
$$

Indeed, for each fixed $U$, we have the isomorphism of abelian groups

$$
\operatorname{Hom}_{\mathbb{Z}}\left(F(U), Z^{d}(U)\right) \cong \operatorname{Hom}_{\mathbb{Z}}\left(\mathbb{Z} \operatorname{Hom}_{\text {spaces }}\left(\Delta_{\text {top }}^{d}, U^{a n}\right), \operatorname{Hom}_{\mathbb{Z}}(F(U), \mathbb{Q} / \mathbb{Z})\right),
$$

which is natural in $U$ in a suitable sense so that we obtain the isomorphism

$$
\operatorname{Hom}_{\text {pshvs }}\left(F, Z^{d}\right) \cong \operatorname{Hom}_{\text {fun }}\left(\mathbb{Z} \operatorname{Hom}_{\text {spaces }}\left(\Delta_{\text {top }}^{d},(-)^{a n}\right), \operatorname{Hom}_{\mathbb{Z}}(F(-), \mathbb{Q} / \mathbb{Z})\right) \text {, }
$$

where the $\operatorname{Hom}_{\text {fun }}$ refers to the abelian group of natural transformations of covariant functors from $S c h / \mathbb{C}$ to abelian groups. Observe that the functor $\mathbb{Z} \operatorname{Hom}_{\text {spaces }}\left(\Delta_{\text {top }}^{d},(-)^{a n}\right)$ can be represented as the following direct limit of representable functors:

$$
\mathbb{Z} \operatorname{Hom}_{\text {spaces }}\left(\Delta_{\text {top }}^{d},(-)^{a n}\right) \cong \underset{\Delta_{\text {top }}^{d} \rightarrow V^{a n}}{\lim _{\text {an }}} \mathbb{Z} \operatorname{Hom}_{S c h}(V,-),
$$


where the indexing category is $\left(\operatorname{Var}^{\Delta_{t o p}^{d}}\right)^{o p}$. Thus we have

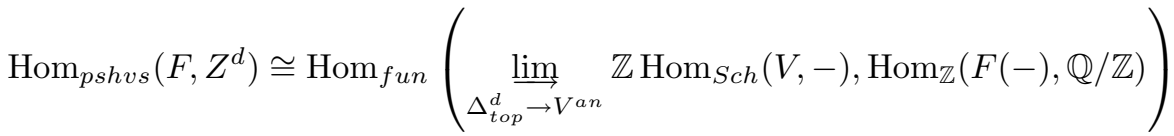

$$
\begin{aligned}
& \cong \varliminf_{\Delta_{\text {top }}^{d} \rightarrow V^{a n}} \operatorname{Hom}_{\text {fun }}\left(\mathbb{Z} \operatorname{Hom}_{S c h}(V,-), \operatorname{Hom}_{\mathbb{Z}}(F(-), \mathbb{Q} / \mathbb{Z})\right) \\
& \left.\cong \varliminf_{\Delta_{\text {top }}^{d} \rightarrow V^{a n}} \operatorname{Hom}_{\mathbb{Z}}(F(V), \mathbb{Q} / \mathbb{Z})\right)
\end{aligned}
$$

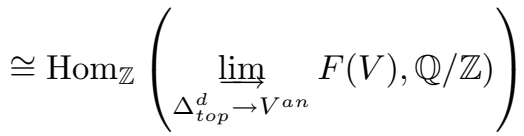

$$
\begin{aligned}
& \left.=\operatorname{Hom}_{\mathbb{Z}}\left(F\left(\Delta_{\text {top }}^{d}\right), \mathbb{Q} / \mathbb{Z}\right)\right) \\
& =F\left(\Delta_{\text {top }}^{d}\right)^{\#} \text {, }
\end{aligned}
$$

where the third isomorphism is given by Yoneda's Lemma.

Using (2.3.1), we see that the cochain complex of abelian groups $\operatorname{Hom}_{p s h v s}\left(F, Z^{\bullet}\right)$ is isomorphic to $F\left(\Delta_{t o p}^{\bullet}\right)^{\#}$, where we regard $F\left(\Delta_{t o p}^{\bullet}\right)$ as a chain complex in the usual manner and duals $(-)^{\#}$ are taken degree-wise. Since $(-)^{\#}$ is a faithful and exact functor, to prove the theorem is suffices to show that the natural map of chain complexes

$$
\operatorname{Hom}_{p s h v s}\left(G, Z^{\bullet}\right) \rightarrow \operatorname{Hom}_{p s h v s}\left(F, Z^{\bullet}\right)
$$

is a quasi-isomorphism. Also using (2.3.1), we see that $Z^{d}$ is an injective object in the category of presheaves of abelian groups on $S c h / \mathbb{C}$, since "evaluation" at $\Delta_{\text {top }}^{d}$ and $(-)^{\#}$ are exact functors. Thus, we may identify $\operatorname{Hom}_{\text {pshvs }}\left(F, Z^{\bullet}\right)$ with $\mathbb{R} \operatorname{Hom}_{D^{+}(\text {pshvs })}\left(F, Z^{\bullet}\right)$ in the derived category of abelian groups, where $D^{+}($pshvs) denotes the derived category of bounded below cochain complexes of presheaves on $S c h / \mathbb{C}$ and $\mathbb{R}$ Hom is the total derived functor.

We now proceed to verify that the cochain complex of presheaves $Z \bullet$ satisfies "naive descent" with respect to the uad-topology; namely, we prove that for any uad-hypercovering $X \bullet \rightarrow X$, the natural map

$$
H_{\text {sing }}^{n}\left(X^{a n}, \mathbb{Q} / \mathbb{Z}\right)=h^{n}\left(Z^{\bullet}(X)\right) \rightarrow h^{n}\left(Z^{\bullet}\left(X_{\bullet}\right)\right)
$$

is an isomorphism for all $n \in \mathbb{Z}$. Since the analytic realization of any complex variety can be triangulated, the singular cohomology of $X^{a n}$ with $\mathbb{Q} / \mathbb{Z}$ coefficients coincides with its sheaf cohomology with values in the constant sheaf $\mathbb{Q} / \mathbb{Z}$ :

$$
H_{\text {sing }}^{n}\left(X^{a n}, \mathbb{Q} / \mathbb{Z}\right) \cong H_{\text {sheaf }}^{n}\left(X^{a n}, \mathbb{Q} / \mathbb{Z}\right) .
$$

Using the spectral sequence for sheaf cohomology of a simplicial space (cf. [D; 5.2.3.2]), this implies that for any simplicial variety $X \bullet$ and integer $n$ the natural map

$$
H_{\text {sheaf }}^{n}\left(Z^{\bullet}\left(X_{\bullet}\right)\right) \rightarrow H_{\text {sheaf }}^{n}\left(X_{\bullet}^{a n}, \mathbb{Q} / \mathbb{Z}\right)
$$

is also an isomorphism. Now, using [D; 5.3.5], we see that the natural map of sheaf cohomology groups

$$
H_{\text {sheaf }}^{n}\left(X^{a n}, \mathbb{Q} / \mathbb{Z}\right) \rightarrow H_{\text {sheaf }}^{n}\left(X_{\bullet}^{a n}, \mathbb{Q} / \mathbb{Z}\right)
$$

is an isomorphism for all $n$ and $u a d$-hypercoverings $X \bullet \rightarrow X$. We thus conclude that (2.3.2) is an isomorphism. 
Let $Z^{\bullet} \rightarrow I^{\bullet} \cdot \bullet$ be an injective resolution in the category of $u a d$-sheaves: a map of bicomplexes of presheaves such that each $\left(Z^{d}\right)_{\text {uad }} \rightarrow I^{d, \bullet}$ is an injective resolution in the category of uad sheaves. Thus, for each fixed $d$, the complex

$$
Z^{d} \rightarrow I^{d, 0} \rightarrow I^{d, 1} \rightarrow \ldots
$$

is exact locally in the uad topology and each $I^{d, e}$ is an injective $u a d$ sheaf. Then for all integers $d, m$, and varieties $X$, the map

$$
\varliminf_{X \bullet \rightarrow X} h^{m}\left(Z^{d}\left(X_{\bullet}\right)\right) \rightarrow h^{m}\left(I^{d, \bullet}(X)\right)
$$

is an isomorphism, where the colimit is indexed by the homotopy category of uad-hypercoverings of $X$ (i.e., objects are $u a d$-hypercoverings and maps are simplicial homotopy classes of maps of augmented simplicial varieties). Combining the isomorphisms (2.3.2) and (2.3.3), we have the isomorphism

$$
h^{m}\left(Z^{\bullet}(X)\right) \cong h^{m}\left(I^{\bullet \bullet \bullet}(X)\right)
$$

for all $m$ and $X$. Thus, $Z^{\bullet} \rightarrow I^{\bullet \bullet \bullet}$ is a quasi-isomorphism of bicomplexes of presheaves.

As with any topology, the forgetful functor takes injective $u a d$-sheaves to injective presheaves, and thus $Z^{\bullet} \rightarrow I^{\bullet}, \bullet$ represents an injective resolution in the category of presheaves of the complex $Z^{\bullet}$ (upon taking total complexes). We thus have a chain of isomorphisms in the derived category of abelian groups

$$
\begin{aligned}
\operatorname{Hom}_{p s h v s}\left(F, Z^{\bullet}\right) & \cong \mathbb{R} \operatorname{Hom}_{D^{+}(p s h v s)}\left(F, Z^{\bullet}\right) \\
& \cong \mathbb{R} \operatorname{Hom}_{D^{+}(p s h v s)}\left(F, I^{\bullet}, \bullet\right) \\
& \cong \operatorname{Hom}_{\text {pshvs }}\left(F, I^{\bullet}, \bullet\right) \\
& \cong \operatorname{Hom}_{\text {uad-shvs }}\left(F_{\text {uad }}, I^{\bullet, \bullet}\right)
\end{aligned}
$$

where the final isomorphism holds since each $I^{d, e}$ is an $u a d$-sheaf. The theorem now follows from the existence of the following commutative square in the derived category of abelian groups

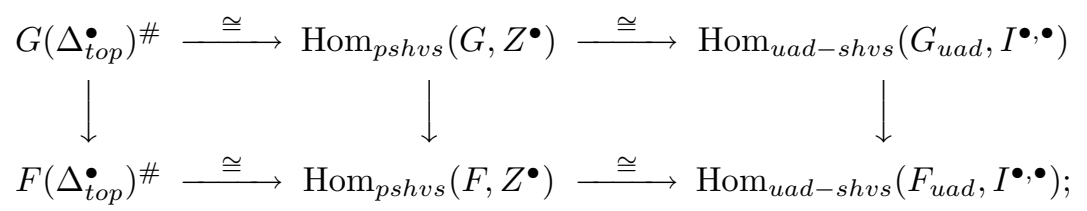

namely, if $F_{\text {uad }} \rightarrow G_{\text {uad }}$ is an isomorphism, then $G\left(\Delta_{\text {top }}\right)^{\#} \rightarrow F\left(\Delta_{\text {top }}^{\bullet}\right)^{\#}$ is a quasi-isomorphism and hence $F\left(\Delta_{\text {top }}\right) \rightarrow G\left(\Delta_{\text {top }}\right)$ is a homotopy equivalence of simplicial abelian groups.

Theorem 2.3 in conjunction with Lemma 2.2 gives us immediately the following useful corollary.

Corollary 2.4. Suppose $F \rightarrow G$ is a map of presheaves of abelian groups on $S c h / \mathbb{C}$ such that the induced map $F_{h} \rightarrow G_{h}$ is an isomorphism. Then $F\left(\Delta_{\text {top }}^{\bullet}\right) \rightarrow G\left(\Delta_{\text {top }}^{\bullet}\right)$ is a homotopy equivalence.

Suslin and Voevodsky have employed pretheories (or presheaves with transfer) in order to establish Suslin rigidity for a large number of presheaves (cf. [SV1]). As shown in [FS], Suslin rigidity remains valid for presheaves such as algebraic $K$-theory which satisfy slightly weaker conditions than that required to be a pretheory - namely, for so-called pseudo-pretheories. The following corollary is thus a direct application of Theorem 2.3 together with Suslin rigidity as developed by [SV1]. 
Corollary 2.5. Let $F$ be a presheaf of abelian groups on $S c h / \mathbb{C}$ whose restriction to $S m / \mathbb{C}$, the category of smooth, quasi-projective complex varieties, is a pseudo-pretheory (cf. [FS; §10]). Suppose there exists an integer $n>0$ such that $n \cdot F=0$. Then $F\left(\Delta_{\text {top }}^{\bullet}\right)$ is homotopy equivalent to the constant simplicial abelian group $F(\operatorname{Spec} \mathbb{C})$.

Proof. As argued in the proof of [FW3; 3.2], the results of [SV1; §4] extend to pseudo-pretheories. In particular, $F(\operatorname{Spec} \mathbb{C}) \cong F_{e t}(X)$ for all smooth varieties $X$, where we view $F($ Spec $\mathbb{C})$ as a constant presheaf. Consequently, the natural map of presheaves

$$
F(\operatorname{Spec} \mathbb{C}) \rightarrow F
$$

induces an isomorphism on associated uad sheaves. Thus, the corollary follows immediately from Theorem 2.3 .

We shall be primarily be concerned with contravariant functors in the context of algebraic topology. For example, we might consider functors taking values in $\Omega$-spectra or simplicial abelian groups, both of which have associated functors to which the following theorem applies.

Theorem 2.6. Let $\theta: F \rightarrow G$ be a map presheaves of group-like $H$-spaces on $S c h / \mathbb{C}$, and assume the simplicial spaces $F\left(\Delta_{\text {top }}\right)$ and $G\left(\Delta_{\text {top }}\right)$ are "good" in the sense of Segal (cf. [Se; A.4]) - for example, assume the functors $F, G: S c h / \mathbb{C} \rightarrow$ Top factor through simplicial sets. If $\theta$ is a weak equivalence locally in the uad-topology, by which we mean for all $q \geq 0$, the uad-sheafification of the map $\pi_{q} F(-) \rightarrow \pi_{q} G(-)$ is an isomorphism, then the induced map of geometric realizations of simplicial spaces

$$
\theta:\left|F\left(\Delta_{t o p}^{\bullet}\right)\right| \rightarrow\left|G\left(\Delta_{\text {top }}^{\bullet}\right)\right|
$$

is a weak equivalence.

Similarly, if the uad-sheafification of the map $\pi_{q}(F(-), \mathbb{Q}) \rightarrow \pi_{q}(G(-), \mathbb{Q})$ is an isomorphism for all $q \geq 0$, then the induced map

$$
\pi_{q}\left(F\left(\Delta_{t o p}^{\bullet}\right), \mathbb{Q}\right) \rightarrow \pi_{q}\left(G\left(\Delta_{\text {top }}^{\bullet}\right), \mathbb{Q}\right)
$$

is an isomorphism for all $q \geq 0$.

Proof. By [BF; B.5] we have a map of convergent spectral sequences from

$$
\pi_{p}\left|d \mapsto \pi_{q} F\left(\Delta_{\text {top }}^{d}\right)\right| \Longrightarrow \pi_{p+q}\left|F\left(\Delta_{t o p}^{\bullet}\right)\right|
$$

to

$$
\pi_{p}\left|d \mapsto \pi_{q} G\left(\Delta_{t o p}^{d}\right)\right| \Longrightarrow \pi_{p+q}\left|G\left(\Delta_{t o p}^{\bullet}\right)\right|,
$$

and thus it suffices to show the map of simplicial abelian groups

$$
\left(d \mapsto \pi_{q} F\left(\Delta_{\text {top }}^{d}\right)\right) \rightarrow\left(d \mapsto \pi_{q} G\left(\Delta_{\text {top }}^{d}\right)\right)
$$

is a homotopy equivalence for all $q \geq 0$. (The "goodness" hypothesis is needed here to ensure $d \mapsto$ $\mid$ Sing. $F\left(\Delta_{\text {top }}^{d}\right) \mid$ is weakly equivalent to $d \mapsto F\left(\Delta_{\text {top }}^{d}\right)$, and similarly for $G$.) This follows immediately from the hypotheses using Theorem 2.3.

The result for rational coefficients follows by the same argument starting with the spectral sequences obtained by tensoring the above ones by $\mathbb{Q}$.

We make explicit the following special case of Theorem 2.6. 
Corollary 2.7. Suppose $F \rightarrow G$ is a map of presheaves of abelian groups (resp., presheaves of group-like $H$ spaces such that $F\left(\Delta_{\text {top }}\right)$ and $G\left(\Delta_{\text {top }}^{\bullet}\right)$ are good) defined on $S c h / \mathbb{C}$ such that $F(U) \rightarrow G(U)$ is an isomorphism (resp., weak equivalence) for all smooth $U$. Then the natural map $F\left(\Delta_{\text {top }}\right) \rightarrow G\left(\Delta_{\text {top }}\right)$ of simplicial abelian groups (resp., simplicial spaces) is a homotopy equivalence (resp. weak homotopy equivalence).

Proof. This follows immediately from Theorem 2.6 and the fact that every variety in $S c h / \mathbb{C}$ is smooth locally in the $h$ topology, a fact which is seen to hold by applying Hironaka's resolution of singularities [H] (or by applying de Jong's theorem on alterations [DeJ]).

As a "demonstration" of the effectiveness of Theorem 2.3, we present a very short proof of one of the main theorems of [FW3].

Theorem 2.8. [FW3; 3.5] For any smooth quasi-projective complex variety $X$ and positive integer $n$, the natural map

$$
K_{q}(X, \mathbb{Z} / n)=\pi_{q}(\mathcal{K}(X), \mathbb{Z} / n) \rightarrow \pi_{q}\left(\mathcal{K}\left(X \times \Delta_{\text {top }}^{\bullet}\right), \mathbb{Z} / n\right)=K_{q}\left(X \times \Delta_{\text {top }}^{\bullet}, \mathbb{Z} / n\right)
$$

is an isomorphism.

Proof. Consider the presheaf of group-like $H$-spaces $G$ on $S c h / \mathbb{C}$ given by

$$
U \mapsto G(U)=\mathcal{K}(X \times U) \wedge M(\mathbb{Z} / n, 0)
$$

where $M(\mathbb{Z} / n, 0)$ is the mod- $n$ Moore spectrum (and we have implicitly taken the zeroth space of the indicated spectrum). Let $F$ be the constant presheaf $U \mapsto \mathcal{K}(X) \wedge M(\mathbb{Z} / n, 0)$. There is a natural map of presheaves $F \rightarrow G$, and for any $q \geq 0$, the induced map on $\pi_{q}$ groups is $K_{q}(X, \mathbb{Z} / n) \rightarrow K_{q}(X \times U, \mathbb{Z} / n)$. Since the restriction of the presheaf $K_{q}(X \times-, \mathbb{Z} / n)$ to $S m / \mathbb{C}$ is a homotopy invariant pseudo-pretheory, the proof of [FW3; 3.2] shows that Suslin rigidity applies to prove that the étale sheafification of $K_{q}(X \times-, \mathbb{Z} / n)$ is the constant sheaf $K_{q}(X, \mathbb{Z} / n)$. The hypotheses of Theorem 2.6 are thus satisfied, and so $F\left(\Delta_{\text {top }}^{\bullet}\right) \rightarrow G\left(\Delta_{\text {top }}^{\bullet}\right)$ is a weak equivalence.

\section{$\S 3$ Reformulation of LaWson homology and morphic COHOMOlOGy}

The formulations of Lawson homology and morphic cohomology have evolved over the past few years. In the case of Lawson homology, these new formulations (e.g., using topological abelian groups of cycles rather than homotopy-theoretic group completions of topological abelian monoids of effective cycles) have merely extended the original definition of Lawson [L] from projective varieties to all quasi-projective varieties without changing the invariants. However, in the case of morphic cohomology, newer formulations with better properties (e.g., [F4]) not only extend the original definition [FL1] to all quasi-projective varieties but also possibly change the invariants for varieties which are not smooth. In the case of smooth varieties, the requirement that morphic cohomology be dual to Lawson homology pins down its values.

In this section, we present yet another formulation of morphic cohomology of a variety $X$, which coincides with the other definitions for smooth varieties but which may differ for singular varieties. Indeed, the theory we introduce here is really a "naive" one, in the sense that Zariski descent (for singular varieties) might not hold. Essentially, the new formulation introduced in this section is given by taking the (naive) motivic cohomology of $X \times \Delta_{t o p}^{\bullet}$ (cf. Corollary 3.5). The advantage this definition had over the others is that it is more easily related to (naive) motivic cohomology and moreover known properties of (naive) motivic cohomology are often transferable to it.

In order to make this discussion somewhat self-contained, we briefly recall the definitions we use for Lawson homology and morphic cohomology. First of all, if $Y$ is a projective (complex) variety given together with an embedding in some projective space $\mathbb{P}^{N}$, then the Chow variety $C_{r, d}(Y)$ is a (not necessarily irreducible) 
closed subvariety of another projective space which has the property that its closed points are in natural oneto-one correspondence with effective $r$-cycles on $Y$ of degree $d$. We write $\mathcal{C}_{r}(Y)$ for the monoid $\coprod_{d \geq 0} C_{r, d}(Y)$ and recall that this monoid is independent of the chosen projective embedding of $Y$. The Lawson homology of the projective variety $Y$ by the formula

$$
L_{r} H_{m}(Y)=\pi_{m-2 r} Z_{r}(Y)
$$

where $Z_{r}(Y)=\left(\mathcal{C}_{r}(Y)^{a n}\right)^{+}$is the "naive group completion" of the topological monoid $\left(\mathcal{C}_{r}(Y)\right)^{a n}$.

More generally, if $Y$ is embedded as a Zariski open subset of a projective variety $\bar{Y}$ with Zariski closed complement $Y_{\infty}$, then $\mathcal{C}_{r}(Y)^{a n}$ is the quotient topological monoid $\mathcal{C}_{r}(\bar{Y})^{a n} / \mathcal{C}_{r}\left(Y_{\infty}\right)^{\text {an }}$ equipped with the quotient topology. Setting

$$
Z_{r}(Y)=\left(\mathcal{C}_{r}(Y)^{a n}\right)^{+}
$$

we may take (3.0.1) to be the definition of Lawson homology for all quasi-projective varieties. It is worth noting that $Z_{r}(Y)$ coincides up to homotopy with $\left(\mathcal{C}_{r}(\bar{Y})^{a n}\right)^{+} /\left(\mathcal{C}_{r}\left(Y_{\infty}\right)^{a n}\right)^{+}=Z_{r}(\bar{Y}) / Z_{r}\left(Y_{\infty}\right)$ (see the proof of $[\mathrm{FG} ; 1.6])$ - that is, one may first group complete and then take quotients.

If $U, V$ are quasi-projective varieties, then $\operatorname{Mor}(U, V)$ is the set of continuous algebraic maps from $U$ to $V$ (cf. [F1]) and $\operatorname{Mor}(U, V)$ is the topological space whose underlying set is $\operatorname{Mor}(U, V)$ and whose topology is defined in [FW1;2.2]. If $X$ is weakly normal and both $X$ and $W$ are projective, then this topology is merely the subspace topology of the mapping complex $\mathcal{M}$ aps $\left(X^{a n}, W^{a n}\right)$ of continuous maps from $X^{a n}$ to $W^{a n}$ provided with the compact open topology. More generally, if $U$ is weakly normal but not necessarily projective, then the topology provided $\operatorname{Mor}(U, W)$ is the "topology of bounded convergence" which is provided with more open sets than that of the compact-open topology (so that convergence in $\operatorname{Mor}(U, W)$ of a sequence $\left\{f_{n}\right\}$ of continuous algebraic maps is stronger than convergence of this sequence in $\mathcal{M}$ aps $\left.\left(Y^{a n}, W^{a n}\right)\right)$. If $U$ is not even weakly normal, then $\operatorname{Mor}(U, W)=\mathcal{M}$ or $\left(U^{w}, W\right)$, where $U^{w} \rightarrow U$ is the so-called weak normalization of $U$. The morphic cohomology of a quasi-projective variety $X$ is defined by the formula

$$
L^{s} H^{n}(X)=\pi_{2 s-n}\left(\operatorname{Mor}\left(X, \mathcal{C}_{0}\left(\mathbb{P}^{s}\right)\right)^{+} / \mathcal{M o r}\left(X, \mathcal{C}_{0}\left(\mathbb{P}^{s-1}\right)\right)^{+}\right)
$$

where $\operatorname{Mor}\left(X, \mathcal{C}_{0}\left(\mathbb{P}^{s}\right)\right)^{+}$denotes the naive group completion of the topological abelian monoid $\mathcal{M o r}\left(X, \mathcal{C}_{0}\left(\mathbb{P}^{s}\right)\right)$.

In order to incorporate the technique developed in Theorem 2.3, we recall the presheaves of equidimensional cycles used in [FV] and [SV2]. If $Y$ is a quasi-projective complex variety and $r$ is a non-negative integer, then there is a unique presheaf $z_{\text {equi }}(Y, r)$ defined on $S c h / \mathbb{C}$ having the following properties: (1) if $U$ is normal, $z_{\text {equi }}(Y, r)(U)$ is the abelian group of cycles on $Y \times U$ freely generated by effective cycles that are equidimensional of relative dimension $r$ over some irreducible component of $U$, and (2) $z_{\text {equi }}(Y, r)$ is a sheaf for the $q f h$ topology. Moreover, the assignment $Y \mapsto z_{\text {equi }}(Y, r)$ is covariantly natural with respect to proper maps and contravariantly functorial (with a degree shift) with respect to flat morphisms. These functorial maps are given, in the case when $U$ is normal by push-forward (along proper maps) and pull-back (along flat morphisms). of cycles. If $Y$ is a projective variety, then the sheaf $z_{\text {equi }}(Y, r)$ coincides with $\operatorname{Mor}\left(-, \mathcal{C}_{r}(Y)\right)^{+}$, which sends a variety $U$ to the (discrete) group completion of the abelian monoid of continuous algebraic maps from $U$ to the Chow monoid $\mathcal{C}_{r}(Y)[\mathrm{F} 4 ; 1.4]$.

As usual, we define the value of the presheaf $z_{\text {equi }}(Y, r)(X \times-)$ on a compact, Hausdorff space $T$ to be

$$
z_{\text {equi }}(Y, r)(X \times T)=\varliminf_{\left(T \rightarrow U^{a n}\right)} z_{\text {equi }}(Y, r)(X \times U) .
$$

where the colimit is indexed by $\left(\operatorname{Var}^{T}\right)^{o p}$. In particular, an element of $z_{\text {equi }}(Y, r)(X \times T)$ is represented by a pair $\left(f: T \rightarrow U^{a n}, \alpha \in z_{\text {equi }}(Y, r)(X \times U)\right)$.

The following proposition relates Lawson homology to the presheaf $z_{\text {equi }}(Y, r)$. 
Proposition 3.1. For any quasi-projective complex variety $Y$, integer $r \geq 0$, and compact Hausdorff space T, there is a map

$$
z_{\text {equi }}(Y, r)(T) \rightarrow \operatorname{Maps}\left(T, Z_{r}(Y)\right)
$$

given by sending an element of the source represented by the pair $\left(f: T \rightarrow U^{a n}, \alpha \in z_{\text {equi }}(Y, r)(U)\right)$ to the function $\left.t \mapsto \alpha\right|_{f(t)} \in z_{\text {equi }}(Y, r)(\operatorname{Spec} \mathbb{C})=Z_{r}(Y)$. This map is contravariantly natural for continuous maps of compact Hausdorff spaces $T^{\prime} \rightarrow T$, contravariantly natural for flat morphism of varieties $Y^{\prime} \rightarrow Y$, and covariantly functorial for proper morphisms of varieties $Y \rightarrow Y^{\prime \prime}$.

In particular, there is a natural map of simplicial sets

$$
z_{\text {equi }}(Y, r)\left(\Delta_{\text {top }}^{\bullet}\right) \rightarrow \operatorname{Sing} \bullet\left(Z_{r}(Y)\right)
$$

If $Y$ is projective, (3.1.2) coincides with the natural equivalence

$$
\left(\operatorname{Sing}_{\bullet}\left(\mathcal{C}_{r}(Y)^{a n}\right)\right)^{+} \stackrel{\sim}{\longrightarrow} \operatorname{Sing} \bullet\left(Z_{r}(Y)\right) .
$$

More generally, if $Y$ is quasi-projective with projective closure $\bar{Y}$ and Zariski closed complement $Y_{\infty}$, then (3.1.2) fits in a map of homotopy fibration sequences

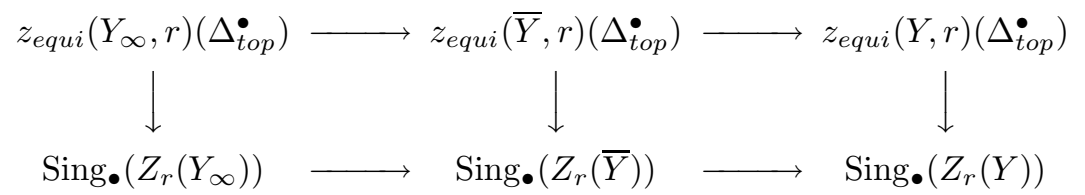

and hence is a homotopy equivalence.

Proof. To verify that (3.1.1) is well defined, we must verify that given a pair $\left(f: T \rightarrow U^{a n}, \alpha \in z_{\text {equi }}(Y, r)(U)\right)$, the function $\left.t \mapsto \alpha\right|_{f(t)}$ is continuous. We claim there is proper, surjective map $p: \tilde{U} \rightarrow U$ with $\tilde{U}$ smooth and an element $\bar{\alpha} \in z_{\text {equi }}(\bar{Y}, r)(\tilde{U})$ such that the restriction of $\tilde{\alpha}$ along $Y \hookrightarrow \bar{Y}$ gives an element in $z_{\text {equi }}(Y, r)(\tilde{U})$ that coincides with $p^{*}(\alpha)$. This holds since the map of presheaves $z_{\text {equi }}(\bar{Y}, r)(-) \rightarrow z_{\text {equi }}(Y, r)(-)$ is surjective locally in the proper $c d h$ topology by [SV2; 4.2.9 and 4.3.1].

Let $\tilde{T}=T \times_{U^{a n}} \tilde{U}^{a n}$ and note that $\tilde{T} \rightarrow T$ is a proper map of topological spaces. The induced map $\tilde{T} \rightarrow \tilde{U}^{a n}$ and the cycle $\bar{\alpha}$ determine a function $\tilde{T} \rightarrow Z_{r}(\bar{Y})$. Since $\bar{\alpha}$ is represented by a difference of morphisms from $\tilde{U} \rightarrow \mathcal{C}_{r}(\bar{Y})$, the function $\tilde{T} \rightarrow Z_{r}(\bar{Y})$ is given by a difference of maps obtained by composing $\tilde{T} \rightarrow \tilde{U}^{a n}$ with elements of $\operatorname{Mor}\left(\tilde{U}, \mathcal{C}_{r}(\bar{Y})\right)$, and hence is continuous. The composition of $\tilde{T} \rightarrow T \rightarrow Z_{r}(Y)$ is easily seen to coincide with the composition of $\tilde{T} \rightarrow Z_{r}(\bar{Y}) \rightarrow Z_{r}(Y)$ and thus is also continuous. Since $\tilde{T} \rightarrow T$ is a quotient space map, it follows that $T \rightarrow Z_{r}(Y)$ must be continuous as well. This is the map $\left.t \mapsto \alpha\right|_{f(t)}$ whose continuity we sought.

The asserted functoriality properties follow from the results of [SV2; §3]. Specifically, $z_{\text {equi }}(Y, r)(-)=$ $z_{\text {equi }}(Y \times-/-, r)$ is a presheaf on $S c h / \mathbb{C}($ see $[\mathrm{SV} 2 ; 3.3 .9])$ from which functoriality in $T$ is immediate. Moreover, pullbacks along flat maps $Y^{\prime} \rightarrow Y$ of relative dimension $s$ (respectively, pushforward along proper morphisms $\left.Y \rightarrow Y^{\prime \prime}\right)$ determine morphisms of presheaves $z_{\text {equi }}(Y, r) \rightarrow z_{r}\left(Y^{\prime}, r+s\right)$ [SV2; 3.6.4] (resp., $z_{\text {equi }}(Y, r) \rightarrow z_{\text {equi }}\left(Y^{\prime \prime}, r\right)$ [SV2; 3.6.3]). Thus, there are pullback and pushforward maps for both the source and target of (3.1.1) as claimed. To prove the functoriality of these maps, it suffices to consider the case where $T$ is a point, in which case it is obvious.

The fact that (3.1.2) is a homotopy equivalence when $Y$ is projective (so that $z_{\text {equi }}(Y, r)$ equals $\operatorname{Mor}\left(-, \mathcal{C}_{r}(Y)\right)^{+}$) follows from Quillen's theorem [FM; App. Q] identifying the level-wise group completion of a simplicial abelian monoid with its homotopy-theoretic group completion.

Finally, to show that (3.1.3) is a map of fibration sequences, we recall from $[\mathrm{FV} ; 5.12,8.1]$ that

$$
z_{\text {equi }}\left(Y_{\infty}, r\right)\left(U \times \Delta^{\bullet}\right) \rightarrow z_{\text {equi }}(\bar{Y}, r)\left(U \times \Delta^{\bullet}\right) \rightarrow z_{\text {equi }}(Y, r)\left(U \times \Delta^{\bullet}\right)
$$


is a fibration sequence whenever $U$ is smooth. Since this composition is the zero map for any variety $U$, there is a natural map from $\left|z_{\text {equi }}\left(Y_{\infty}, r\right)\left(U \times \Delta^{\bullet}\right)\right|$ to the homotopy fiber of

$$
\left|z_{\text {equi }}(\bar{Y}, r)\left(U \times \Delta^{\bullet}\right)\right| \rightarrow\left|z_{\text {equi }}(Y, r)\left(U \times \Delta^{\bullet}\right)\right| .
$$

Consequently, Corollary 2.7 implies that

$$
z_{\text {equi }}\left(Y_{\infty}, r\right)\left(\Delta_{\text {top }}^{\bullet} \times \Delta^{\bullet}\right) \rightarrow z_{\text {equi }}(\bar{Y}, r)\left(\Delta_{\text {top }}^{\bullet} \times \Delta^{\bullet}\right) \rightarrow z_{\text {equi }}(Y, r)\left(\Delta_{\text {top }}^{\bullet} \times \Delta^{\bullet}\right)
$$

is also a homotopy fibration sequence. By homotopy invariance (cf. [FW3; 1.2]), we conclude that the upper row of (3.1.3) is a homotopy fibration sequence. The lower row is a fibration sequence by [FG; 1.6].

The following corollary is merely a restatement of the conclusion of Proposition 3.1.

Corollary 3.2. For any quasi-projective complex variety $Y$, there exists a natural isomorphism

$$
\pi_{m-2 r}\left(z_{\text {equi }}(Y, r)\left(\Delta_{\text {top }}^{\bullet}\right)\right) \stackrel{\cong}{\rightrightarrows} L_{r} H_{m}(Y)
$$

where $L_{r} H_{m}(Y)$ is the Lawson homology of $Y$.

In order to provide a similar interpretation for morphic cohomology, we extend the construction of the map (3.1.1) to a bivariant context (with $Y$ projective).

Proposition 3.3. If $Y$ is a projective complex variety and $X$ is a normal quasi-projective complex variety, then there is a map

$$
z_{\text {equi }}(Y, r)(X \times T) \rightarrow \operatorname{Maps}\left(T, \mathcal{M o r}\left(X, \mathcal{C}_{r}(Y)\right)^{+}\right)
$$

given by sending $(f, \alpha)$ to $\left.t \mapsto \alpha\right|_{f(t)} \in z_{\text {equi }}(Y, r)(X)=\operatorname{Mor}\left(X, \mathcal{C}_{r}(Y)\right)^{+}$. This map is contravariantly natural for continuous maps of compact Hausdorff spaces $T^{\prime} \rightarrow T$, contravariantly functorial for all morphisms of varieties $X^{\prime} \rightarrow X$, contravariantly functorial for all flat morphisms of varieties $Y^{\prime} \rightarrow Y$, and covariantly functorial for all proper morphisms of varieties $Y \rightarrow Y^{\prime \prime}$.

Moreover, this map is compatible with that of Proposition 3.1 in the sense that for any projective complex variety $Y$, quasi-projective smooth variety $X$ of pure dimension $d$, integer $r \geq 0$, and compact Hausdorff space $T$, the diagram

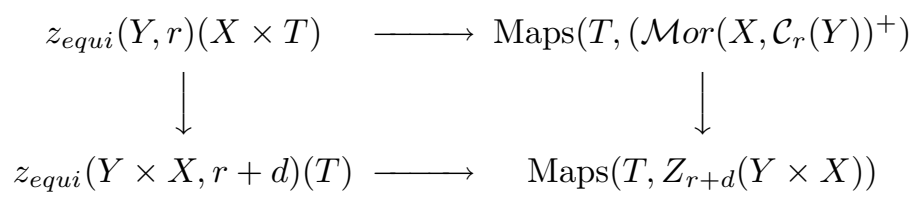

commutes, where the top arrow is (3.3.1), the bottom arrow is given by (3.1.1), and the two vertical arrows are given by the evident inclusions of cycles.

Proof. Once again, we must prove the continuity of the map sending $(f, \alpha)$ to $\left.t \mapsto \alpha\right|_{f(t)}$. Given $(f: T \rightarrow$ $\left.U^{a n}, \alpha \in z_{\text {equi }}(Y, r)(X \times U)\right)$, let $\tilde{U} \rightarrow U$ denote the normalization of $U, \tilde{\alpha}$ denote the pullback of $\alpha$ to $z_{\text {equi }}(Y, r)(X \times \tilde{U})$, and $\tilde{T}$ denote $T \times_{U^{a n}} \tilde{U}^{a n}$. The precomposition of $T \rightarrow Z_{r}(Y)$ with $\tilde{T} \rightarrow T$ coincides with the function $\tilde{T} \rightarrow Z_{r}(Y)$ determined by the pair $\left(\tilde{f}: \tilde{T} \rightarrow \tilde{U}^{a n}, \tilde{\alpha}\right)$. Since $X \times \tilde{U}$ is normal, $\tilde{\alpha}$ is represented by a difference of elements of $\operatorname{Mor}\left(X \times \tilde{U}, \mathcal{C}_{r}(Y)\right)$. The continuity of $\tilde{T} \rightarrow Z_{r}(Y)$, and hence of $T \rightarrow Z_{r}(Y)$ follows. Functoriality is verified as for (3.1.1) using the results of [SV2; §3].

To show (3.3.2) commutes, note that the left hand vertical arrow arises from the inclusion of presheaves $z_{\text {equi }}(Y, r)(X \times-) \hookrightarrow z_{\text {equi }}(Y \times X, r+d)(-)$. (The definition of these presheaves given in [SV2; §3] makes it clear that each is a sub-presheaf of a common presheaf $\operatorname{Cycl}(Y \times X / \operatorname{Spec} \mathbb{C}, r+d)_{\mathbb{Q}}$.) In particular, this arrow is natural in $T$. We have shown that the horizontal arrows are also natural in $T$ and the right-hand vertical arrow is clearly natural in $T$. It therefore suffices to establish the commutativity of (3.2.2) in the special case where $T$ is the one-point space. In this case the result is obvious.

We now give a bivariant generalization of Proposition 3.1. 
Theorem 3.4. Given a smooth quasi-projective complex variety $X$ and a smooth projective complex variety $Y$, the natural map of simplicial sets

$$
z_{\text {equi }}(Y, 0)\left(X \times \Delta_{\text {top }}^{\bullet}\right) \rightarrow \operatorname{Sing} \bullet\left(\mathcal{M o r}\left(X, \mathcal{C}_{0}(Y)\right)^{+}\right)
$$

is a homotopy equivalence.

Proof. We may assume $X$ is connected, say of dimension $d$. By Proposition 3.3, the diagram

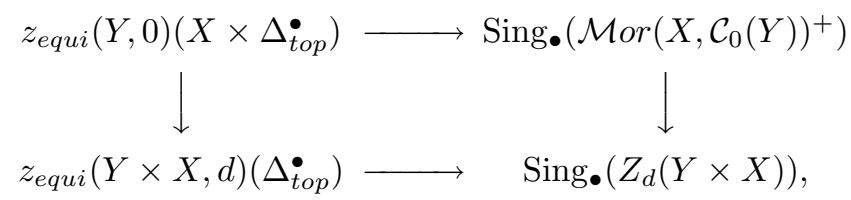

commutes. The lower horizontal map of (3.4.1) is a homotopy equivalence by Proposition 3.1, whereas the right vertical map of (3.4.1) is a homotopy equivalence by duality of morphic cohomology and Lawson homology for smooth varieties [F3; 5.2].

We recall from $[\mathrm{FV} ; 8.1,8.2]$ that the natural map

$$
z_{\text {equi }}(Y, 0)\left(-\times \Delta^{\bullet} \times X\right) \rightarrow z_{\text {equi }}(X \times Y, d)\left(-\times \Delta^{\bullet}\right)
$$

of presheaves induces a homotopy equivalence on all smooth varieties. Thus,

$$
z_{\text {equi }}(Y, 0)\left(X \times \Delta_{\text {top }}^{\bullet} \times \Delta^{\bullet}\right) \rightarrow z_{\text {equi }}(X \times Y, d)\left(\Delta_{\text {top }}^{\bullet} \times \Delta^{\bullet}\right)
$$

is also a homotopy equivalence by Corollary 2.7. Homotopy invariance $z_{\text {equi }}(V, n)\left(U \times \Delta_{\text {top }}^{\bullet} \times-\right.$ ) (cf. [FW3; $1.2])$ thus implies that the left vertical map of (3.4.1) is a homotopy equivalence.

Consequently, we conclude that the upper horizontal map of (3.4.1) is a homotopy equivalence as asserted.

Let $\mathbb{P}^{q}=\operatorname{Proj} \mathbb{C}\left[x_{0}, \ldots, x_{q}\right]$ and $\mathbb{P}^{q-1} \hookrightarrow \mathbb{P}^{q}$ be the inclusion into the first $q$ homogeneous coordinates. We let $z_{\text {equi }}\left(\mathbb{P}^{q / q-1}, r\right)$ denote the cokernel of the map of presheaves

$$
z_{\text {equi }}\left(\mathbb{P}^{q-1}, r\right) \rightarrow z_{\text {equi }}\left(\mathbb{P}^{q}, r\right)
$$

induced by the closed immersion $\mathbb{P}^{q-1} \hookrightarrow \mathbb{P}^{q}$. In other words, we have

$$
z_{\text {equi }}\left(\mathbb{P}^{q / q-1}, r\right)(U)=\operatorname{Mor}\left(U, \mathcal{C}_{r}\left(\mathbb{P}^{q}\right)\right)^{+} / \operatorname{Mor}\left(U, \mathcal{C}_{r}\left(\mathbb{P}^{q-1}\right)\right)^{+} .
$$

Restriction to the open subscheme $\mathbb{A}^{q}=\mathbb{P}^{q}-\mathbb{P}^{q-1}$ defines a morphism of presheaves $z_{\text {equi }}\left(\mathbb{P}^{q / q-1}, r\right) \rightarrow$ $z_{\text {equi }}\left(\mathbb{A}^{q}, r\right)$.

We summarize our reformulation of morphic cohomology (for smooth varieties) in the following corollary.

Corollary 3.5. For any smooth, quasi-projective complex variety $X$, the natural chain of maps

$$
\begin{gathered}
z_{\text {equi }}\left(\mathbb{A}^{q}, 0\right)\left(X \times \Delta^{\bullet} \times \Delta_{\text {top }}^{\bullet}\right) \rightarrow z_{\text {equi }}\left(\mathbb{A}^{q}, 0\right)\left(X \times \Delta_{\text {top }}^{\bullet} \leftarrow z_{\text {equi }}\left(\mathbb{P}^{q / q-1}, 0\right)\left(X \times \Delta_{\text {top }}^{\bullet}\right)\right. \\
\left.\left.\rightarrow \operatorname{Sing} \bullet\left(\mathcal{M o r}\left(X, \mathcal{C}_{0}\left(\mathbb{P}^{q}\right)\right)^{a n}\right)\right)^{+} / \operatorname{Sing} \bullet\left(\operatorname{Mor}\left(X, \mathcal{C}_{0}\left(\mathbb{P}^{q-1}\right)\right)^{a n}\right)\right)^{+}
\end{gathered}
$$

consists of homotopy equivalences. The induced map on $\pi_{j}$,

$$
H_{\mathcal{M}}^{2 q-j}\left(X \times \Delta_{\text {top }}^{\bullet}, \mathbb{Z}(q)\right) \stackrel{\cong}{\rightrightarrows} L^{q} H^{2 q-j}(X),
$$

identifies the motivic cohomology of $X \times \Delta_{\text {top }}^{\bullet}$ with the morphic cohomology of $X$.

Proof. The first map is a homotopy equivalence by homotopy invariance. The fact that the second map is a homotopy equivalence follows by using the homotopy equivalence (3.4.2), the homotopy fibration sequence (3.1.3), and the 5-Lemma. The fact that the third map is a homotopy equivalence follows from Theorem 3.4 and another application of the 5-Lemma. 


\section{$\S 4$ The total Segre Class map and Chern character for semi-topological $K$-theory}

In Section 1 we considered the total Segre map from the (naive) algebraic $K$-theory space to the multiplicative motivic cohomology space,

$$
\text { Seg }: \mathcal{K}^{\text {naive }}(X) \rightarrow \mathcal{H}_{\text {mult }}(X)
$$

and showed it is a rational equivalence for $X$ smooth. In section 3 , we implicitly saw how to formulate the analogous map from semi-topological $K$-theory to morphic cohomology as

$$
\operatorname{Seg}^{\text {sst }}: \mathcal{K}\left(X \times \Delta_{\text {top }}^{\bullet}\right) \rightarrow \mathcal{H}_{\text {mult }}\left(X \times \Delta_{\text {top }}^{\bullet}\right) .
$$

Moreover, in section 2 we provided a criterion which enables us to verify that a map such as $S e g^{s s t}$ when restricted to smooth schemes is a homotopy equivalence. Thus, the pieces are all in place to establish Theorem 4.2, that $S e g^{s s t}$ is a rational equivalence. Similarly, we established in section 1 that the Chern character $\mathrm{ch}^{\text {alg }}$ associated to $\mathrm{Seg}^{\text {alg }}$ is a multiplicative rational isomorphism. With some more effort, the techniques we have developed will enable us to show in Theorem 4.7 that

$$
c h^{s s t}: \bigoplus_{n} K_{n}\left(X \times \Delta_{t o p}^{\bullet}\right)_{\mathbb{Q}} \rightarrow \bigoplus_{q, n} L^{q} H^{2 q-n}(X)_{\mathbb{Q}}
$$

is an isomorphism of graded rings.

Recall that if $F:(S c h / \mathbb{C})^{o p} \rightarrow($ sets $)$ is a presheaf of sets, then we define the simplicial set

$$
F\left(\Delta_{\text {top }}^{\bullet}\right)=\left(n \mapsto F\left(\Delta_{\text {top }}^{n}\right)\right)
$$

where for any topological space $T$, we define $F(T)=\varliminf_{T \rightarrow U^{a n}} F(U)$ where the colimit is indexed by $\left(\operatorname{Var}^{T}\right)^{o p}$. In particular, this construction is used to define the semi-topological $K$-theory space $\mathcal{K}^{s s t}(X)=$ $\mathcal{K}\left(X \times \Delta_{\text {top }}^{\bullet}\right)$ as we recall at the beginning of the following definition.

Definition 4.1. For any quasi-projective variety $X$, the singular semi-topological $K$-theory of $X$ is the spectrum (or infinite loop space) $\mathcal{K}^{\text {sst }}(X)$ associated to the $\left|\mathcal{I}\left(\Delta^{\bullet}\right)\right|$-space $\mid H o m\left(X \times \Delta_{\text {top }}^{\bullet}\right.$, Grass) $\mid[\mathrm{FW} 4 ; 2.1]$. We sometimes use the more cumbersome but more suggestive notation $\mathcal{K}\left(X \times \Delta_{\text {top }}^{\bullet}\right.$ in place of $\mathcal{K}^{\text {sst }}(X)$, and we frequently say "semi-topological K-theory" instead of "singular semi-topological K-theory".

Similarly, the (singular) semi-topological motivic groups are defined by the formula

$$
H_{\text {sst }}^{n}(X, \mathbb{Z}(q))=H_{\mathcal{M}}^{n}\left(X \times \Delta_{\text {top }}^{\bullet}, \mathbb{Z}(q)\right)=\pi_{2 q-n}\left|z_{\text {equi }}\left(\mathbb{A}^{q}, 0\right)\left(X \times \Delta_{\text {top }}^{\bullet}\right)\right| .
$$

Define $\mathcal{H}_{\text {mult }}\left(X \times \Delta_{\text {top }}^{\bullet}\right)=\mathcal{H}_{\text {mult }}^{\text {sst }}(X)$ to be the spectrum (or infinite loop space) associated to the $\left|\mathcal{I}\left(\Delta^{\bullet}\right)\right|$ space $\operatorname{Hom}\left(X \times \Delta_{\text {top }}^{\bullet} \text {, Chow }\right)_{1}^{+}$, and the semi-topological total Segre map

$$
S e g^{s s t}: \mathcal{K}^{s s t}(X) \rightarrow \mathcal{H}_{\text {mult }}^{\text {sst }}(X)
$$

to be the map on associated spectra (or infinite loop spaces) induced by the map

$$
\text { cyc }: \operatorname{Hom}\left(X \times \Delta_{\text {top }}^{\bullet}, \operatorname{Grass}\right) \rightarrow \operatorname{Hom}\left(X \times \Delta_{\text {top }}^{\bullet}, \text { Chow }\right)_{1}^{+} \text {. }
$$

of $\left|\mathcal{I}\left(\Delta^{\bullet}\right)\right|$-spaces.

For any $n, q \geq 0$, we define the semi-topological Segre classes

$$
s_{n, q}^{s s t}: K_{n}^{s s t}(X) \rightarrow L^{q} H^{2 q-n}(X),
$$

to be the composition

$$
\begin{aligned}
K_{n}^{s s t}(X) \stackrel{(-)^{*}}{\longrightarrow} K_{n}^{s s t}(X) & \stackrel{\pi_{n}\left(\text { Seg }^{\text {sst }}\right)}{\longrightarrow} \pi_{n} \mathcal{H}_{\text {mult }}^{\text {sst }}(X) \\
& \cong \bigoplus_{q} H_{s s t}^{2 q-n}(X, \mathbb{Z}(q)) \rightarrow H_{\text {sst }}^{2 q-n}(X, \mathbb{Z}(q)) \stackrel{\cong}{\longrightarrow} L^{q} H^{2 q-n}(X)
\end{aligned}
$$

where the first indicated isomorphism is given by Proposition 1.4 and the second by Corollary 3.5.

In the following theorem, we make explicit the fact that the group structure on $\pi_{*} \mathcal{H}_{\text {mult }}^{\text {sst }}(X)$ is not the evident group structure on morphic cohomology in homotopy degree 0 . This reflects the fact that the total Segre class is multiplicative: $s(x+y)=s(x) s(y)$. 
Theorem 4.2. For any smooth, quasi-projective complex variety $X$, the semi-topological Segre map

$$
\operatorname{Seg}^{s s t}: \mathcal{K}^{s s t}(X) \rightarrow \mathcal{H}_{\text {mult }}^{\text {sst }}(X)
$$

is a rational equivalence.

Equivalently, for each $n \geq 0$, the semi-topological rational total Segre class

$$
s_{n, *}^{s s t}: K_{n}^{s s t}(X)_{\mathbb{Q}} \rightarrow \bigoplus_{q} L^{q} H^{2 q-n}(X, \mathbb{Q}),
$$

is an isomorphism of graded groups. For $n=0$, the group structure on the right is given by the join pairing and can be viewed as the product of $\mathbb{Z}\left[\pi_{0}(X)\right]$ times the multiplicative group of units with leading term 1:

$$
\{1\} \times \bigoplus_{q \geq 1} L^{q} H^{2 q-n}(X, \mathbb{Q}) \subset \bigoplus_{q \geq 0} L^{q} H^{2 q-n}(X, \mathbb{Q}) .
$$

Proof. Since $\mathrm{Seg}$ is a map of group-like H-spaces, Theorem 2.6 implies that to prove $\mathrm{Seg}^{\text {sst }}$ is a rational isomorphism (for $X$ smooth) it suffices to prove that

$$
\text { Seg }: \mathcal{K}(X \times-) \rightarrow \mathcal{H}_{\text {mult }}(X \times-)
$$

is a rational isomorphism when restricted to smooth varieties $U$. This is given by Theorem 1.10.

Since $s_{n, *}^{s s t}$ differs from $S e g_{n}=\pi_{n} S e g$ by an automorphism of $K_{n}^{s s t}(X)$, the fact that $s_{n, *}^{s s t}$ is a rational isomorphism follows immediately from the fact that $S e g$ is a rational equivalence.

In [FW4], we established semi-topological real $K$-theory for varieties defined over the real numbers $\mathbb{R}$. Extending the construction of Section 2, if $F: S c h / \mathbb{R} \rightarrow($ sets $)$ is a contravariant functor defined on quasiprojective real varieties, we define

$$
F_{\mathbb{R}}\left(\Delta_{\text {top }}^{\bullet}\right)=\left(n \mapsto F_{\mathbb{R}}\left(\Delta_{\text {top }}^{n}\right)\right)
$$

where for any topological space $T$ we set $F_{\mathbb{R}}(T)$ equal to the set $\varliminf_{T \rightarrow U(\mathbb{R})} F(U)$. Here, the indexing category of the colimit is $\left(\operatorname{Var}_{\mathbb{R}}^{T}\right)^{o p}$, where an object of $\operatorname{Var}_{\mathbb{R}}^{T}$ is a continuous map $T \rightarrow U(\mathbb{R})$ (where $U$ is a real variety and $U(\mathbb{R})$ is the analytic space of real points) and morphisms in $\operatorname{Var}_{\mathbb{R}}^{T}$ are given by morphisms of real varieties satisfying the evident commutativity condition. 4.1 .

The following is simply the real analogue of the definition of $\mathcal{K}^{s s t}(X)=\mathcal{K}\left(X \times \Delta_{\text {top }}^{\bullet}\right)$ given in Definition

Definition 4.3. [FW4; 2.1] For any quasi-projective real variety $X$, we define

$$
\mathcal{K} \mathbb{R}^{s s t}(X)=\mathcal{K}\left(X \times_{\mathbb{R}} \Delta_{\text {top }}^{\bullet}\right)
$$

to be the spectrum (or infinite loop space) associated to the $\left|\mathcal{I}\left(\Delta_{\mathbb{R}}^{\bullet}\right)\right|$-space $\left|\operatorname{Hom}_{\mathbb{R}}\left(X \times_{\mathbb{R}} \Delta_{\text {top }}^{\bullet}, \operatorname{Grass}_{\mathbb{R}}\right)\right|$.

Similarly, for such a quasi-projective real variety $X$, we define

$$
\mathcal{H}_{\text {mult }, \mathbb{R}}^{\text {sst }}(X)=\mathcal{H}_{\text {mult }}\left(X \times_{\mathbb{R}} \Delta_{\text {top }}^{\bullet}\right)
$$

to be the spectrum (or infinite loop space) associated to the $\left|\mathcal{I}\left(\Delta_{\mathbb{R}}^{\bullet}\right)\right|$-space $\mid \operatorname{Hom}_{\mathbb{R}}\left(X \times_{\mathbb{R}} \Delta_{\text {top }} \text {, Chow }{ }_{\mathbb{R}}\right)_{1}^{+} \mid$.

We define

$$
S e g_{\mathbb{R}}: \mathcal{K} \mathbb{R}^{s s t}(X) \rightarrow \mathcal{H}_{\text {mult }, \mathbb{R}}^{\text {sst }}(X)
$$

to be the map of spectra associated to the map of $\left|\mathcal{I}\left(\Delta_{\mathbb{R}}^{\bullet}\right)\right|$-spaces

$$
\text { cyc : }\left|\operatorname{Hom}_{\mathbb{R}}\left(X \times_{\mathbb{R}} \Delta_{\text {top }}^{\bullet}, \operatorname{Grass}_{\mathbb{R}}\right)\right| \rightarrow\left|\operatorname{Hom}_{\mathbb{R}}\left(X \times_{\mathbb{R}} \Delta_{\text {top }}^{\bullet}, \operatorname{Chow}_{\mathbb{R}}\right)_{1}^{+}\right| .
$$

Thanks to the foundations of real semi-topological $K$-theory established in [FW4], we easily conclude the real analogue of Theorem 4.2. 
Corollary 4.4. Let $X$ be a smooth quasi-projective real variety. Then the total Segre class induces a rational homotopy equivalence

$$
\operatorname{Seg}_{\mathbb{R}}: \mathcal{K} \mathbb{R}^{s s t}(X) \rightarrow \mathcal{H}_{m u l t, \mathbb{R}}^{s s t}(X) .
$$

Thus, for each $n \geq 0$, the semi-topological total real Segre class

$$
s_{\mathbb{R}, n, *}^{s s t}: K \mathbb{R}_{n}^{s s t}(X) \rightarrow \bigoplus_{q} L^{q} H \mathbb{R}^{2 q-n}(X),
$$

is a rational isomorphism of graded groups.

Proof. Let $X_{\mathbb{C}}$ denote $X \times_{\mathbb{R}}$ Spec $\mathbb{C}$ and let $\pi: X_{\mathbb{C}} \rightarrow X$ be the canonical map. In [FW4; 5.4], a natural transfer map

$$
\pi_{*}: \mathcal{K}^{s s t}\left(X_{\mathbb{C}}\right) \sim \mathcal{K} \mathbb{R}^{s s t}\left(X_{\mathbb{C}}\right) \rightarrow \mathcal{K} \mathbb{R}_{n}^{s s t}(X)
$$

is constructed; in [FW4; 5.6], it is shown that the composition of this transfer map with the natural pullback map

$$
\pi^{*}: \mathcal{K} \mathbb{R}^{s s t}(X) \rightarrow \mathcal{K} \mathbb{R}^{s s t}\left(X_{\mathbb{C}}\right) \sim \mathcal{K}^{s s t}\left(X_{\mathbb{C}}\right)
$$

is weakly homotopic to multiplication by 2 . Since we have a similar result for $\mathcal{H}_{\text {mult }}^{\text {sst }}(-)$ in place of $\mathcal{K}^{s s t}(-)$, we conclude that upon inverting the prime 2, the map $S e g_{\mathbb{R}}$ is a retract of the rational equivalence

$$
\operatorname{Seg}: \mathcal{K}^{s s t}\left(X_{\mathbb{C}}\right) \rightarrow \mathcal{H}_{m u l t}^{s s t}\left(X_{\mathbb{C}}\right)
$$

Thus, $S e g_{\mathbb{R}}$ is also a rational equivalence.

The assertion about the total real Segre class follows immediately from the definition of $L^{q} H \mathbb{R}^{2 q-n}(X)$ given in [FW4] and the argument at the end of the proof of Theorem 4.2.

We define the semi-topological Chern character in terms of semi-topological Segre classes using the same polynomials as we used for the Chern character on algebraic $K$-theory (preceding Theorem 1.12):

$$
c h_{n, q}^{s s t}=P_{q}\left(s_{n, 0}^{s s t}, \ldots, s_{n, q}^{s s t}\right),
$$

where for $n>0$, products of the form $s_{n, i}^{s s t} s_{n, j}^{s s t}$ with $i, j \geq 1$ are trivial.

To prove $c h^{s s t}$ is multiplicative, we employ a dimension shifting technique similar to that of Section 1 which is suitable for use with semi-topological $K$-theory and morphic cohomology. For a finite oriented simplicial complex $M$, define the (singular) semi-topological $K$-theory of $X \times|M|$, written $\mathcal{K}^{s s t}(X \times|M|)$, to be the homotopy theoretic group completion of the $\left|\mathcal{I}\left(\Delta^{\bullet}\right)\right|$-space $\mid \operatorname{Hom}\left(X \times|M| \times \Delta_{\text {top }}^{\bullet}\right.$, Grass $) \mid$. In particular, $K_{0}^{s s t}(X \times|M|)$ is the group completion of the abelian monoid $\pi_{0} \mid \operatorname{Hom}\left(X \times|M| \times \Delta_{\text {top }}^{\bullet}\right.$, Grass $) \mid$ - equivalently, $K_{0}^{s s t}(X \times|M|)$ is isomorphic to the direct limit

$$
\varliminf_{|M| \rightarrow U^{a n}} K_{0}^{s s t}(X \times U)
$$

where the colimit is indexed by the category $\operatorname{Var}^{|M|}$. Observe that there is a map of $\left|\mathcal{I}\left(\Delta^{\bullet}\right)\right|$-spaces

$$
\left|\operatorname{Hom}\left(X \times|M| \times \Delta_{\text {top }}^{\bullet}, \operatorname{Grass}\right)\right| \rightarrow \operatorname{Maps}\left(|M|,\left|\operatorname{Hom}\left(X \times \Delta_{\text {top }}^{\bullet}, \operatorname{Grass}\right)\right|\right)
$$

and hence a map on associated spectra. By [FW2; 3.4], the group-like $H$-space obtained by taking the 0 -th space of the spectrum associated to the $\left|\mathcal{I}\left(\Delta^{\bullet}\right)\right|$-space $\mid \operatorname{Hom}\left(X \times \Delta_{\text {top }}^{\bullet}\right.$, Grass $) \mid$ is naturally weakly equivalent to the mapping telescope associated to a certain endomorphism of $\mid \operatorname{Hom}\left(X \times \Delta_{\text {top }}^{\bullet}\right.$, Grass $) \mid$. It follows that 
the 0 -th space of the spectrum associated to $\operatorname{Maps}\left(|M|, \mid \operatorname{Hom}\left(X \times \Delta_{\text {top }}\right.\right.$, Grass $\left.) \mid\right)$ is naturally homotopy equivalent to

$$
\mathcal{M a p s}\left(M, \mathcal{K}^{s s t}(X)\right)
$$

We therefore have a map

$$
\mathcal{K}^{s s t}(X \times|M|) \rightarrow \mathcal{M a p s}\left(|M|, \mathcal{K}^{s s t}(X)\right)
$$

which is natural up to weak homotopy in both $X$ and $M$. Our semi-topological dimension shifting technique given by the assertion that this map is a weak equivalence. This is proven in Theorem 4.7 below, but first we establish a necessary technical result.

Let $\mathcal{S}(M)$ denote the simplicial set associated to $M$. More precisely, $\mathcal{S}(M)_{n}$ is the collection of order preserving maps from $[n]=\{0<1<\cdots<n\}$ to $V$ (the vertex set of $M$ ) and the face and degeneracy maps for $\mathcal{S}(M)$ are given by composition in the evident manner. Observe that $|\mathcal{S}(M)|$, the geometric realization of the simplicial set $\mathcal{S}(M)$, is naturally homeomorphic to $|M|$. For any presheaf of sets $\mathcal{F}$ on $S c h / \mathbb{C}$ and any simplicial set $S_{\bullet}$, there is a natural map $\mathcal{F}\left(\left|S_{\bullet}\right|\right) \rightarrow \operatorname{Hom}_{\text {s.sets }}\left(S_{\bullet}, \mathcal{F}\left(\Delta_{\text {top }}^{\bullet}\right)\right)$, defined in the following manner. Note that $\operatorname{Hom}_{\text {s.sets }}\left(S_{\bullet}, \mathcal{F}\left(\Delta_{\text {top }}\right)\right)$ is the equalizer of the pair of maps

$$
\prod_{[n], s \in S_{n}} \mathcal{F}\left(\Delta_{\text {top }}^{n}\right) \rightrightarrows \prod_{\left[n_{1}\right] \rightarrow\left[n_{0}\right], s \in S_{n_{0}}} \mathcal{F}\left(\Delta_{\text {top }}^{n_{1}}\right) .
$$

The map $\mathcal{F}\left(\left|S_{\bullet}\right|\right) \rightarrow \operatorname{Hom}_{\text {s.sets }}\left(S_{\bullet}, \mathcal{F}\left(\Delta_{\text {top }}^{\bullet}\right)\right)$ is induced by the collection of natural maps $\Delta_{\text {top }}^{n} \rightarrow\left|S_{\bullet}\right|$ associated to each pair $[n], s \in S_{n}$. In particular, we have a natural map

$$
\mathcal{F}(|M|) \rightarrow \operatorname{Hom}_{\text {s.sets }}\left(\mathcal{S}(M), \mathcal{F}\left(\Delta_{\text {top }}^{\bullet}\right)\right) .
$$

Lemma 4.5. Let $\mathcal{F}$ be a presheaf of sets on $S c h / \mathbb{C}$ satisfying the condition that for every pullback square of finitely generated $\mathbb{C}$-algebras

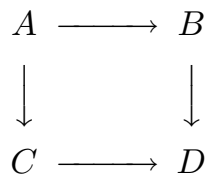

in which all four maps are surjections (so that the associated square of spectra is a push-out diagram of closed immersions of affine varieties), the induced diagram

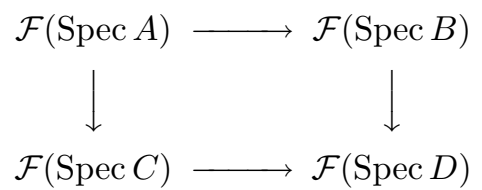

is also a pullback. Then the natural map

$$
\mathcal{F}(|M|) \rightarrow \operatorname{Hom}_{\text {s.sets }}\left(\mathcal{S}(M), \mathcal{F}\left(\Delta_{\text {top }}^{\bullet}\right)\right)
$$

is a bijection for any finite, oriented simplicial complex $M$.

Proof. We proceed by double induction on the dimension $d$ of $M$ and the number of maximal simplices of $M$. If $M$ is a $d$-simplex, then (4.5.1) is obviously a bijection. Otherwise, let $M=M_{1} \cup M_{2}$ be a nontrivial decomposition of $M$ into two subsimplicial complexes, and consider the map from the square

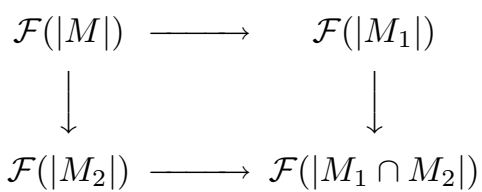


to the square

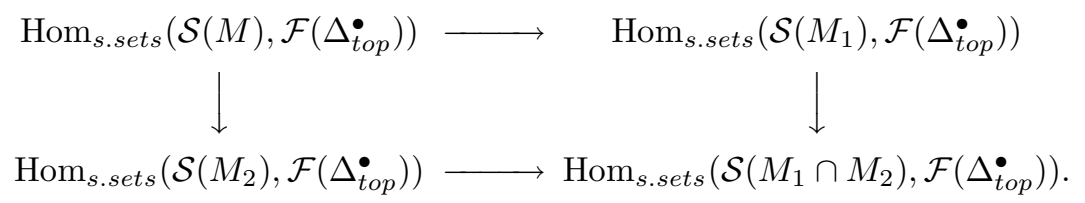

The latter square is a pullback square, since the simplicial sets $\mathcal{S}(M), \mathcal{S}\left(M_{1}\right), \mathcal{S}\left(M_{2}\right)$, and $\mathcal{S}\left(M_{1} \cap M_{2}\right)$ form a pushout square. If (4.5.2) is also a pullback, then our claim follows by induction (since $M_{1}, M_{2}$, and $M_{1} \cap M_{2}$ are each either of smaller dimension or have fewer maximal simplices than $M$ ).

By [FW2; 4.2], for any compact CW complex $N$, we have $\mathcal{F}(N) \cong \varliminf_{A \subset \mathcal{C}(N)} \mathcal{F}(\operatorname{Spec} A)$, where $A$ ranges over finitely generated $\mathbb{C}$-subalgebras of $\mathcal{C}(N)$, the $\mathbb{C}$-algebra of continuous complex-valued functions on $N$. In light of the hypothesis on $\mathcal{F}$, it suffices to prove that the pullback square

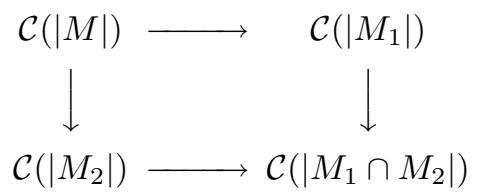

(in which all maps are surjections) is a filtered direct limit of pullback squares of the form

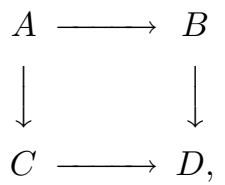

in which all maps are surjections and $A, B, C$, and $D$ are finitely generated $\mathbb{C}$-subalgebras of $\mathcal{C}(|M|), \mathcal{C}\left(\left|M_{1}\right|\right)$, $\mathcal{C}\left(\left|M_{2}\right|\right)$, and $\mathcal{C}\left(\left|M_{1} \cap M_{2}\right|\right)$, respectively. To see this, note that given any finitely generated subalgebra $D$ of $\mathcal{C}\left(\left|M_{1} \cap M_{2}\right|\right)$, by taking inverse images of chosen finite set of generators of $D$, we may form a square of the form (4.5.4).

The following proposition provides us with the means to interpret the group $K_{n}^{s s t}(X)$ in terms of the group $K_{0}^{s s t}(X \times|M|)$, thereby playing a role for semi-topological $K$-theory similar to that played by Proposition 1.7 for algebraic $K$-theory. Observe that $K_{0}^{s s t}\left(X \times|M|_{k}\right)$ is not isomorphic to $\left[|M|, \mathcal{K}^{s s t}(X)\right]$ - if it were, then one would be able to deduce that $K_{n}(X) \rightarrow K_{n}^{s s t}(X)$ is a surjection for all $n$, which is absurd. Hence, one must replace $|M|_{k}$ with $|M|$ in the context of semi-topological $K$-theory in order to obtain a form of dimension shifting.

Proposition 4.6. For any quasi-projective complex variety $X$ and any finite, oriented simplicial complex $M$, the natural map

$$
\mathcal{K}^{s s t}(X \times|M|) \rightarrow \mathcal{M a p s}\left(|M|, \mathcal{K}^{s s t}(X)\right)
$$

is a weak homotopy equivalence. Consequently, there is an isomorphism

$$
K_{0}^{s s t}(X \times|M|) \cong\left[|M|, \mathcal{K}^{s s t}(X)\right]
$$

which is natural in both $X$ and $M$.

Proof. Choose a surjection $\mathcal{O}_{X}^{n} \rightarrow \mathcal{L}$ with $\mathcal{L}$ a very ample line bundle on $X$. Let $\alpha$ be the endomorphism of the presheaf $\operatorname{Hom}(X \times-$, Grass $)$ defined at a fixed $U$,

$$
\alpha: \operatorname{Hom}(X \times U, \text { Grass }) \rightarrow \operatorname{Hom}(X \times U, \text { Grass }),
$$


by sending a quotient object $\mathcal{O}_{X \times U}^{\infty} \rightarrow \mathcal{E}$ to the quotient object determined by the composition of

$$
\mathcal{O}_{X \times U}^{\infty} \cong \mathcal{O}_{X \times U}^{n} \oplus \mathcal{O}_{X \times U}^{\infty} \rightarrow \mathcal{L} \oplus \mathcal{E} .
$$

Here, the isomorphism is the evident "shift" map. Now define $\operatorname{Hom}(X \times- \text {, Grass })^{\infty}$ to be the direct limit of presheaves

$$
\varliminf(\operatorname{Hom}(X \times-, \text { Grass }) \stackrel{\alpha}{\longrightarrow} \operatorname{Hom}(X \times-, \text { Grass }) \stackrel{\alpha}{\longrightarrow} \ldots) .
$$

Then for any affine variety $U$, the simplicial set $d \mapsto \operatorname{Hom}\left(X \times U \times \Delta_{\text {top }}^{d} \text {, Grass }\right)^{\infty}$ is weakly homotopy equivalent to space $\mathcal{K}^{\text {sst }}(X \times U)$ by [FW4; 7.3]. Since $\operatorname{Hom}\left(X \times|M| \times \Delta_{\text {top }}^{\bullet} \text {, Grass }\right)^{\infty}$ is the direct limit of simplicial sets of the form $\operatorname{Hom}\left(X \times U \times \Delta_{\text {top }}^{\bullet} \text {, Grass }\right)^{\infty}$ for $U$ affine by [FW2; 4.2], we have a weak homotopy equivalence

$$
\mathcal{K}^{s s t}(X \times|M|) \sim \mid \operatorname{Hom}\left(X \times|M| \times \Delta_{\text {top }}^{\bullet}, \text { Grass }\right)^{\infty} \mid .
$$

It therefore suffices to prove that the natural map

$$
\left|\operatorname{Hom}\left(X \times|M| \times \Delta_{\text {top }}^{\bullet}, \operatorname{Grass}\right)^{\infty}\right| \rightarrow \mathcal{M a p s}\left(|M|, \mid \operatorname{Hom}\left(X \times \Delta_{\text {top }}^{\bullet}, \text { Grass }\right)^{\infty} \mid\right)
$$

is a weak homotopy equivalence. Since this map is a direct limit of copies of the map

$$
\left|\operatorname{Hom}\left(X \times|M| \times \Delta_{\text {top }}^{\bullet}, \operatorname{Grass}\right)\right| \rightarrow \mathcal{M a p s}\left(|M|,\left|\operatorname{Hom}\left(X \times \Delta_{\text {top }}^{\bullet}, \operatorname{Grass}\right)\right|\right),
$$

it suffices to prove this latter map is a weak homotopy equivalence.

By Lemma 4.5, the natural map

$$
\operatorname{Hom}(X \times|M|, \operatorname{Grass}) \rightarrow \operatorname{Hom}_{\text {s.sets }}\left(\mathcal{S}(M), \operatorname{Hom}\left(X \times \Delta_{\text {top }}^{\bullet}, \operatorname{Grass}\right)\right)
$$

is a bijection for any finite, oriented simplicial complex $M$. This shows, in particular, that the simplicial set $\operatorname{Hom}\left(X \times \Delta_{\text {top }}^{\bullet}\right.$, Grass) satisfies the Kan extension condition. (For note that if $\Lambda \subset \Delta^{n}$ denotes the simplicial set obtained from $\Delta^{n}$ by removing any one face, then $\Lambda$ and $\Delta^{n}$ can both be realized as finite, oriented simplicial complexes and

$$
\operatorname{Hom}(X \times|\Delta|, \text { Grass }) \rightarrow \operatorname{Hom}(X \times|\Lambda| \text {, Grass })
$$

is clearly surjective.) Moreover, since $M \times \Delta^{d}$ is also a finite, oriented simplicial complex for all $d$, we obtain an isomorphism

$$
\left(d \mapsto \operatorname{Hom}\left(X \times|M| \times \Delta_{\text {top }}^{d}, \operatorname{Grass}\right)\right) \cong\left(d \mapsto \operatorname{Hom}_{\text {s.sets }}\left(\mathcal{S}(M) \times \Delta^{d}, \operatorname{Hom}\left(X \times \Delta_{\text {top }}^{\bullet}, \operatorname{Grass}\right)\right)\right)
$$

of simplicial sets. The result now follows from the fact that the Kan condition implies the natural map

$$
\left|d \mapsto \operatorname{Hom}_{\text {s.sets }}\left(\mathcal{S}(M) \times \Delta^{d}, \operatorname{Hom}\left(X \times \Delta_{\text {top }}^{\bullet}, \operatorname{Grass}\right)\right)\right| \rightarrow \mathcal{M a p s}\left(|M|,\left|\operatorname{Hom}\left(X \times \Delta_{\text {top }}^{\bullet}, \operatorname{Grass}\right)\right|\right)
$$

is a weak homotopy equivalence.

We can now prove the following main theorem.

Theorem 4.7. The semi-topological Chern character associated to Seg ${ }^{\text {sst }}$,

$$
c h^{s s t}: \bigoplus_{n} K_{n}^{s s t}(X) \rightarrow \bigoplus_{q, n} L^{q} H^{2 q-n}(X, \mathbb{Q}),
$$


is a natural transformation of graded-ring valued functors on the category of smooth, quasi-projective complex varieties.

Moreover, ch $^{\text {sst }}$ induces a rational isomorphism for all $X \in S m / \mathbb{C}$ :

$$
c h^{s s t}: \bigoplus_{n} K_{n}^{s s t}(X)_{\mathbb{Q}} \stackrel{\cong}{\longrightarrow} \bigoplus_{q, n} L^{q} H^{2 q-n}(X, \mathbb{Q}) \text {. }
$$

Finally, for any $X \in S m / \mathbb{C}$, the diagram

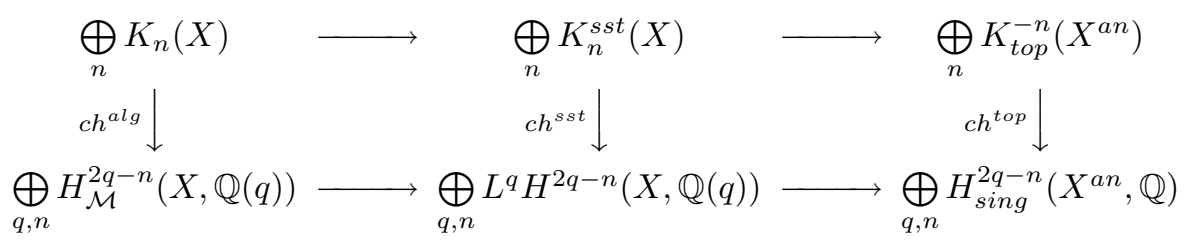

commutes.

Remark. As in Theorem 1.12, observe that $L^{q} H^{2 q-n}(X)=0$ for $q-n>d=\operatorname{dim}(X)$, so that for a fixed $n$, the target of $\mathrm{ch}_{n}^{\text {semi }}$ is a finite sum of cohomology groups. Indeed, using duality [F3; 5.2] and the Dold-Thom Theorem, we have

$$
L^{q} H^{2 q-n}(X) \cong L^{q} H^{2 q-n}\left(X \times \mathbb{A}^{q-d}\right) \cong L_{0} H_{n}\left(X \times \mathbb{A}^{q-d}\right) \cong H_{n}^{B M}\left(X \times \mathbb{A}^{q-d}\right) \cong H_{n-2 q+2 d}^{B M}(X)=0
$$

whenever $q>d+n$ and $n \geq 0$ (for then $n-2 q+2 d<0$ ).

Proof of 4.7. A slight modification of the proof of Theorem 1.12 for $\mathrm{ch}^{\text {alg }}$ also applies to show $c h^{\text {sst }}$ is a ring homomorphism. More specifically, let $X$ and $Y$ be smooth, quasi-projective complex varieties and suppose $M$ and $N$ are finite, oriented simplicial complexes with $|M| \cong S^{m}$ and $N \cong S^{n}$. Suppose $f_{1}:|M| \rightarrow W^{a n}$ and $g_{1}:|N| \rightarrow Z^{a n}$ are continuous maps with $W$ and $Z$ arbitrary (quasi-projective) complex varieties, and suppose $f_{2}: X \times W \rightarrow U$ and $g_{2}: Y \times Z \rightarrow V$ are morphisms of varieties with $U$ and $V$ smooth. The pair $f=\left(f_{1}, f_{2}\right)$ induces the map

$$
f^{\#}: K_{0}(U) \rightarrow K_{m}^{s s t}(X)
$$

defined as the composition of

$$
K_{0}(U) \stackrel{f_{2}^{*}}{\longrightarrow} K_{0}(X \times Y) \stackrel{\left(i d \times f_{1}\right)^{*}}{\longrightarrow} K_{0}(X \times|M|) \rightarrow K_{0}^{s s t}(X \times|M|) \stackrel{\cong}{\longrightarrow}\left[|M|, \mathcal{K}^{s s t}(X)\right] \rightarrow K_{m}^{s s t}(X),
$$

where the isomorphism is given by Proposition 4.6 and the last map is the canonical surjection determined by $|M| \cong S^{m}$. Similarly, the pair $g=\left(g_{1}, g_{2}\right)$ defines the map $g^{\#}: K_{0}(V) \rightarrow K_{n}^{s s t}(Y)$. As in the proof of Theorem 1.12, we obtain the analogue of (1.12.1), the commutative square

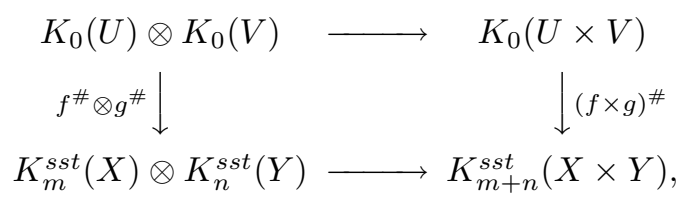

where $f \times g=\left(f_{1} \times g_{1}, f_{2} \times g_{2}\right)$.

Likewise, we obtain the semi-topological analogue of (1.12.2), and the commutativity of the first square of Proposition 1.9 remains valid (for the same reasons) in the semi-topological context. Naturality then gives us that the square

$$
\begin{array}{rlr}
K_{m}^{s s t}(X) \otimes K_{n}^{s s t}(Y) & \longrightarrow & K_{m+n}^{s s t}(X \times Y) \\
c h_{m}^{s s t} \otimes c h_{n}^{s s t} \downarrow & c h_{m+n}^{s s t} \downarrow \\
\bigoplus_{p} L^{p} H^{2 p-m}(X) \otimes \bigoplus_{q} L^{q} H^{2 q-n}(X) & \longrightarrow \bigoplus_{r} L^{r} H^{2 r-m-n}(X)
\end{array}
$$


commutes upon restriction to the image of

$$
K_{0}(U) \otimes K_{0}(V) \stackrel{f^{\#} \otimes g^{\#}}{\longrightarrow} K_{m}^{s s t}(X) \otimes K_{n}^{s s t}(Y) .
$$

It therefore suffices to show that as $W, Z, U, V, f_{1}, f_{2}, g_{1}$, and $g_{2}$ vary over all possibilities, the union of these images form a generating set. Every element of $K_{0}(X \times|M|)$ lies in the image of $\left(i d \times f_{1}\right)^{*}$ for some $f_{1}:|M| \rightarrow W^{a n}$ (and similarly for $\left.Y \times|N|\right)$. Moreover, as with any (possibly singular) variety, every element of $K_{0}(X \times W)$ lies in the image of $f_{2}^{*}$ for some $f_{2}: X \times W \rightarrow U$ with $U$ smooth (and similarly for $Y \times Z$ ). Thus $c h^{s s t}$ is a ring homomorphism. The fact that it induces a rational isomorphism follows from Theorem 4.2 .

To prove the commutativity of (4.7.1), recall that the total Segre maps determine a commutative diagram of infinite loop spaces denoted (0.4) in the introduction ([FW2; 6.12], [FW4; 8.6])

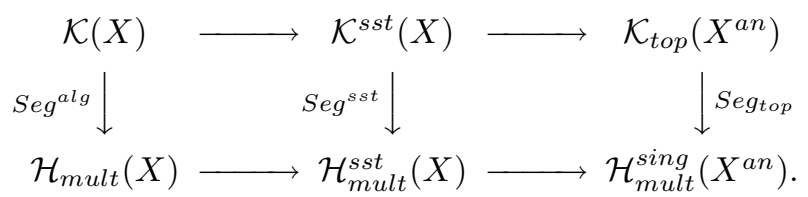

Since the total Segre classes arise from the Segre maps (after precomposition with talking duals) and since the Chern characters arise from the total Segre classes using the same universal polynomials in all three contexts (algebraic, semi-topological, and topological), we conclude the commutativity of (4.7.1).

In view of Corollary 4.3, Theorem 4.7 has the following corollary.

Corollary 4.8. Let $\mathrm{ch}_{\mathbb{R}}^{\text {sst }}$ be the real semi-topological Chern character defined in terms of the real semitopological Segre classes of Corollary 4.3 using the same polynomials as those determining ch $^{\text {alg }}$ in terms of Segre classes $s_{*}$. Then for any smooth quasi-projective real variety $X$,

$$
c h_{\mathbb{R}}^{s s t}: \bigoplus_{n} K \mathbb{R}_{n}^{s s t}(X) \rightarrow \bigoplus_{n, q} L^{q} H \mathbb{R}^{2 q-n}(X)_{\mathbb{Q}}
$$

is a map of graded rings which is a rational isomorphism.

\section{$\S 5$ Riemann-Roch and Filtrations}

In this final section, we obtain some consequences of our rational isomorphism between semi-topological $K$-theory and morphic cohomology. These applications depend strongly upon the refined assertion (Theorem 4.7) that the Chern character is a ring homomorphism. Riemann-Roch asserts the compatibility of semi-topological $K$-theory and morphic cohomology with respect to proper push-forward maps. As we observed earlier in [FW4], the general Riemann-Roch theorem of I. Panin and A. Smirnov [PS] (see also [P]) specializes to give a Riemann-Roch theorem for semi-topological $K$-theory thanks to Theorem 4.7. Using the multiplicativity of the Chern character, we also establish the relationship between multiplication by "the Bott element" in $K$-theory and application of the "s-operation" in morphic cohomology. For example, we see that we can re-interpret a filtration on rational cohomology (related to the rational Hodge filtration) in strictly $K$-theoretic terms.

Following Panin-Smirnov [PS], let $S m \mathcal{O} p$ denote the category of pairs $(X, U)$ of smooth complex quasiprojective varieties with $U$ an open subscheme of $X$, and define a cohomology theory to be a functor $A^{*}$ from $S m \mathcal{O} p$ to the category of graded abelian groups,

$$
A^{*}: S m \mathcal{O} p \rightarrow(G r A b),
$$


satisfying the axioms of [PS; 2.0.1]. (Briefly, these axioms require the existence of expected long exact sequences, homotopy invariance, and Nisnevich excision.) Such a functor is a multiplicative cohomology theory if there are external cup-product type pairings $A^{m}(X, U) \otimes A^{n}(Y, V) \rightarrow A^{m+n}(X \times Y, X \times V \cup U \times Y)$ satisfying the axioms given in [PS; 2.3.1] (which require, briefly, associativity, existence of 1 , and the expected compatibility with boundary maps). Finally, an orientation for such a functor is a choice of a Chern class $c_{1}(L) \in A(X)$ for each line bundle $L$ on a smooth variety $X$ such that the axioms of [PS; 3.2.1] hold (which require naturality, the expected bundle formula for $X \times \mathbb{P}^{1}$, and that $\left.c_{1}\left(\mathcal{O}_{X}\right)=0\right)$.

By embedding the category $S m / \mathbb{C}$ of smooth complex quasi-projective varieties into $S m \mathcal{O} p$ in the natural way (sending $X$ to $(X, \emptyset)$ ), we may restrict a multiplicative cohomology theory $A$ to a functor $\left.A\right|_{S m / \mathbb{C}}$ : $S m / \mathbb{C} \rightarrow($ GrRings $)$ from smooth quasi-projective complex varieties to graded rings.

Recall from Thomason-Trobaugh [TT; 7.4] that given a closed subvariety $Z$ of a quasi-projective variety $X$, the algebraic $K$-theory of $X$ with supports in $Z$ is given by the spectrum $\mathcal{K}(X)^{Z}$ defined from the category of perfect complexes on $X$ supported in $Z$. In [FW4; App. A], the Thomason-Trobaugh construction is extended to the semi-topological setting by defining $\mathcal{K}^{s s t}(X)^{Z}$ to be the geometric realization of $d \mapsto \mathcal{K}\left(X \times \Delta_{\text {top }}^{d}\right)^{Z}$,

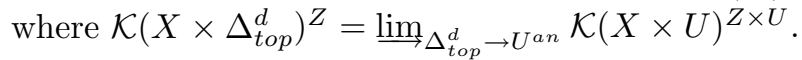

Similarly, the motivic cohomology of $X$ (for $X$ smooth) with supports in a closed subscheme is defined using Zariski sheaf hypercohomology with supports by the formula

$$
H_{\mathcal{M}}^{n}(X, \mathbb{Z}(q))^{Z}=\mathbb{H}_{Z}^{n}(X, \mathbb{Z}(q))=\mathbb{H}_{Z}^{n}\left(X, z_{\text {equi }}\left(\mathbb{A}^{q}, 0\right)\left(-\times \Delta^{\bullet}\right)\right)
$$

Equivalently, for $X$ smooth, Theorem 1.3 gives the natural isomorphism

$$
\left.H_{\mathcal{M}}^{n}(X, \mathbb{Z}(q))^{Z} \cong h^{n}\left(\text { cone }\left[z_{\text {equi }}\left(\mathbb{A}^{q}, 0\right)\left(X \times \Delta^{\bullet}\right)\right) \rightarrow z_{\text {equi }}\left(\mathbb{A}^{q}, 0\right)\left((X-Z) \times \Delta^{\bullet}\right)\right][-1]\right) .
$$

We define the semi-topological analogue for a smooth variety $X$ and closed subscheme $Z$ by the expected formula:

$$
\begin{aligned}
H_{\text {sst }}^{n}(X, \mathbb{Z}(q))^{Z} & =\mathbb{H}_{Z}^{n}\left(X, z_{\text {equi }}\left(\mathbb{A}^{q}, 0\right)\left(-\times \Delta_{\text {top }}^{\bullet}\right)\right) \\
& \left.\cong h^{n}\left(\operatorname{cone}\left[z_{\text {equi }}\left(\mathbb{A}^{q}, 0\right)\left(X \times \Delta_{\text {top }}^{\bullet}\right)\right) \rightarrow z_{\text {equi }}\left(\mathbb{A}^{q}, 0\right)\left((X-Z) \times \Delta_{\text {top }}^{\bullet}\right)\right][-1]\right)
\end{aligned}
$$

By Theorem 3.4, the group $H_{s s t}^{n}(X, \mathbb{Z}(q))^{Z}$ is naturally isomorphic to

$\pi_{2 q-n}\left(\right.$ htpy-fiber $\left.\left[\operatorname{Mor}\left(X, \mathcal{C}\left(\mathbb{P}^{q}\right)\right)^{+} / \operatorname{Mor}\left(X, \mathcal{C}\left(\mathbb{P}^{q-1}\right)\right)^{+} \rightarrow \operatorname{Mor}\left(X-Z, \mathcal{C}\left(\mathbb{P}^{q}\right)\right)^{+} / \mathcal{M o r}\left(X-Z, \mathcal{C}\left(\mathbb{P}^{q-1}\right)\right)^{+}\right]\right)$

which is the definition of morphic cohomology with supports given in [F4; 2$]$. In particular, we have $H_{s s t}^{n}(X, \mathbb{Z}(q))^{X} \cong L^{q} H^{n}(X)$.

Proposition 5.1. Each of the functors from $S m \mathcal{O} p$ to graded rings given by sending $(X, U) \in S m \mathcal{O} p$ to

$$
K_{-*}(X)^{X-U}, \quad K_{-*}^{s s t}(X)^{X-U}, \quad \text { and } \quad K_{\text {top }}^{*}\left(X^{a n}, U^{a n}\right)
$$

determines a multiplicative cohomology theory in the sense of Panin-Smirnov. Moreover, an orientation for each of these theories is given by defining the first Chern class of a line bundle $L$ to be $c_{1}(L)=\left[\mathcal{O}_{X}\right]-\left[\mathcal{O}_{L} \vee\right] \in$ $K_{0}(X)$ (respectively, its images in $K_{0}^{s s t}(X)$ and $\left.K_{\text {top }}^{0}\left(X^{a n}\right)\right)$ ).

Similarly, each of the three functors defined by sending $(X, U)$ to

$$
\bigoplus_{q} H_{\mathcal{M}}^{2 q+*}(X, \mathbb{Z}(q))^{X-U}, \quad \bigoplus_{q} H_{\text {sst }}^{2 q+*}(X, \mathbb{Z}(q))^{X-U}, \quad \text { and } \quad H_{\text {sing }}^{*}\left(X^{a n}, U^{a n} ; \mathbb{Z}\right)
$$


determines a multiplicative cohomology theory which is oriented by defining the first Chern class associated to a line bundle $L \rightarrow X$ by the formula

$$
c_{1}(L)=D(L) \in C H^{1}(X) \cong H_{\mathcal{M}}^{2}(X, \mathbb{Z}(1))
$$

(respectively, the images of $D(L)$ in $H_{\text {sst }}^{2}(X, \mathbb{Z}(1))$ and $H_{\text {sing }}^{2}(X)$ ), where $D(L)$ is the Weil divisor associated to $L$.

Proof. The Panin-Smirnov axioms for $K$-theory and motivic cohomology are verified in [PS; $§ 3.6]$. (Note that Panin-Smirnov use a different indexing convention for viewing motivic cohomology as an oriented multiplicative cohomology theory, but the axioms remain valid with our convention.) For semi-topological $K$-theory, these axioms are verified in [FW4; 9.3] (in the more general context of real singular semi-topological $K$-theory).

For the theory $(X, U) \mapsto A^{p}(X, U)=\bigoplus_{q} H_{s s t}^{2 q+p}(X, \mathbb{Z}(q))^{X-U}$, the required long exact sequences are immediate from the definition of hypercohomology with supports, while homotopy invariance and Nisnevich excision follow from Theorem 2.6 and the fact that these properties hold for motivic cohomology with supports. Thus, $A^{*}$ is a cohomology theory (cf. [PS; 2.0.1]). The multiplicative pairings are induced by the pairings of complexes of presheaves

$z_{\text {equi }}\left(\mathbb{A}^{q}, 0\right)\left(-\times \Delta_{\text {top }}^{\bullet}\right) \otimes z_{\text {equi }}\left(\mathbb{A}^{r}, 0\right)\left(-\times \Delta_{\text {top }}^{\bullet}\right) \rightarrow z_{\text {equi }}\left(\mathbb{A}^{r+q}, 0\right)\left(-\times \Delta_{\text {top }}^{\bullet} \times \Delta_{\text {top }}^{\bullet}\right) \rightarrow z_{\text {equi }}\left(\mathbb{A}^{r+q}, 0\right)\left(-\times \Delta_{\text {top }}^{\bullet}\right)$

induced by taking Cartesian products of cycles and pulling back along the diagonal $\Delta_{\text {top }}^{\bullet} \hookrightarrow \Delta_{\text {top }}^{\bullet} \times \Delta_{\text {top }}^{\boldsymbol{\bullet}}$. This pairing is clearly unital and associative up to homotopy, and the required compatibility of multiplication with the boundary maps is a formal consequence of the properties of hypercohomology with supports. Thus the axioms of [PS; 2.3.1] hold, making $A^{*}$ a multiplicative cohomology theory. Finally, to show that the indicated definition of a first Chern class makes $A^{*}$ into an oriented theory, one uses Theorem 2.6 and the fact that the required bundle formula holds for motivic cohomology. (Naturality and the fact that $c_{1}\left(\mathcal{O}_{X}\right)=0$ are obvious.)

The Panin-Smirnov axioms for the topological theories follow from well known properties of topological $K$-theory and singular cohomology.

As formulated by Grothendieck, the Riemann-Roch Theorem is a statement about the compatibility of covariant push-forward maps of contravariant functors related by a natural transformation, $\phi: A \rightarrow B$. In the work of Panin-Smirnov, push-forward maps $f$ ! for proper maps $f$ are constructed for any oriented multiplicative cohomology theory $A^{*}$. Suppose $\phi: A^{*} \rightarrow B^{*}$ is a so-called ring operation by which we mean $\phi$ is a natural transformation of the graded ring valued functors $\left.\left.A^{*}\right|_{S m / \mathbb{C}} \rightarrow B^{*}\right|_{S m / \mathbb{C}}$ defined on $S m / \mathbb{C}$ (cf. [PS; 2.3.3]). Then Panin and Smirnov define the inverse Todd genus of $\phi$ by the formula

$$
i t d_{\phi}(t)=\phi_{\infty}(u) / t \in B[[t]]
$$

where

$$
\phi_{\infty}: A^{*}(\operatorname{Spec} \mathbb{C})[[u]]=A^{*}\left(\mathbb{P}^{\infty}\right) \rightarrow B^{*}\left(\mathbb{P}^{\infty}\right)=B^{*}(\operatorname{Spec} \mathbb{C})[[t]]
$$

is defined as the evident inverse limit of maps. If $i t d_{\phi}(t)$ is a unit of $B^{*}(\operatorname{Spec} \mathbb{C})[[t]]$ (a property which holds for all the cases in which we are interested), then the Todd genus of $\phi$ is defined to be

$$
t d_{\phi}(t)=1 / i t d_{\phi}(t)=t / \phi_{\infty}(u) .
$$

In this situation, Panin-Smirnov establish a quite general Riemann-Roch result relating the push-forward maps $f_{!}$, the ring operation $\phi$, and the Todd genus of $\phi$ in the expected manner [PS; 5.1.4], [P; 2.5.4].

In the theorem below, $t d_{c h}$ is the Todd genus of the Chern character, and is given explicitly by the formula $t d_{c h}=t /\left(1-e^{-t}\right)$ (cf. [PS; 5.2.1]). For an arbitrary vector bundle $E$, the class $t d_{c h}(E)$ is defined as in [PS; 5.1.2]. 
Theorem 5.2. Let $f: Y \rightarrow X$ be a proper map of smooth quasi-projective complex varieties and let $f_{\text {! }}$ denote the push-forward maps as defined in [PS; 4.4.1] for each of the oriented multiplicative cohomology theories of Proposition 5.1. Then we have the following commutative diagrams:

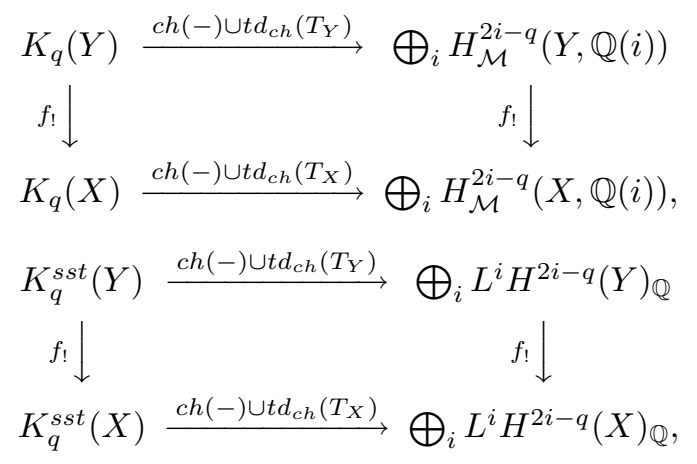

and

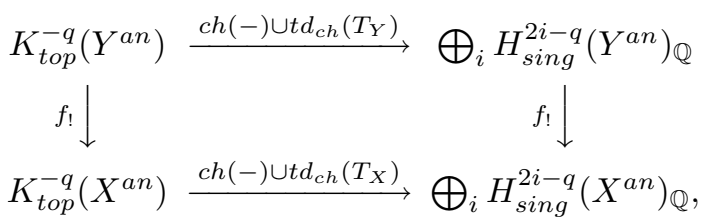

where $T_{Y}$ and $T_{X}$ denote the tangent bundles of $X$ and $Y$.

Moreover, the evident double-cube diagram formed by these commutative squares using the natural transformations $K_{*}(-) \rightarrow K_{*}^{s s t}(-) \rightarrow K_{\text {top }}^{-*}\left((-)^{\text {an }}\right)$ and $H_{\mathcal{M}}^{*}(-, \mathbb{Z}(*)) \rightarrow L^{*} H^{*}(-) \rightarrow H_{\text {sing }}^{*}\left((-)^{\text {an }}\right)$ commutes.

Proof. Using Proposition 5.1, Theorem 1.12, Theorem 4.7, and the fact that the topological Chern character is known to be a ring homomorphism, the theorem is an immediate consequence of the Panin-Smirnov Riemann-Roch Theorem [PS; 5.1.4] (see also [P; 2.5.4]).

The final assertion follows from [PS; 5.1.6] (see also [P; 2.5.6]), since the natural transformations $K_{*}(-) \rightarrow$ $K_{*}^{\text {sst }}(-) \rightarrow K_{\text {top }}^{-*}\left((-)^{a n}\right)$ and $H_{\mathcal{M}}^{*}(-, \mathbb{Z}(*)) \rightarrow L^{*} H^{*}(-) \rightarrow H_{\text {sing }}^{*}\left((-)^{a n}\right)$ preserve Chern classes by construction.

The following proposition makes more explicit the push-forward maps $f$ ! occurring in the commutative squares of Theorem 5.2 for algebraic $K$-theory and morphic cohomology.

Proposition 5.3. Let $f: Y \rightarrow X$ be a proper map of smooth quasi-projective complex varieties. Then the map $f_{!}: K_{*}(Y) \rightarrow K_{*}(X)$ occurring in Theorem 5.2 coincides with composition of

$$
K_{*}(Y) \stackrel{\cong}{\longrightarrow} G_{*}(Y) \stackrel{f_{*}}{\longrightarrow} G_{*}(X) \stackrel{\cong}{\leftrightarrows} K_{*}(X),
$$

where $G_{*}(-)$ denotes the $K$-theory of the exact category of coherent sheaves on a variety and $f_{*}$ is defined by push-forward of coherent sheaves.

The push-forward map f! for morphic cohomology occurring in Theorem 5.2 coincides with the composition of

$$
\bigoplus_{q} L^{q} H^{2 q-*}(Y) \stackrel{\cong}{\longrightarrow} \bigoplus_{r} L_{r} H_{2 r+*}(Y) \stackrel{f_{*}}{\longrightarrow} \bigoplus_{r} L_{r} H_{2 r+*}(X) \cong \bigoplus_{q} L^{q} H^{2 q-*}(Y),
$$

defined by composing the natural pushforward map in Lawson homology with the duality isomorphisms (see [FL2; 5.4] and [F3; 5.3]).

Proof. Each of the theories $X \mapsto K_{-*}(X)$ and $X \mapsto \bigoplus_{q} L^{q} H^{2 q+*}(X)$ is a "ring cohomology pretheory" in the sense of [P; 1.1.1]. Moreover, the assignment $f \mapsto f$ ! (where $f$ ranges over proper morphisms of 
smooth, quasi-projective complex varieties) defined in [PS; 4.4.1] is a "perfect integration" in the sense of $\left[\mathrm{P} ;\right.$ 1.1.6]. For each of the two theories in question, let $f_{*}$ denote the push-forward map associated to a proper morphism $f$ described in the statement of the proposition. By [P; 1.1.11], it suffices to prove that the assignment $f \mapsto f_{*}$ is also a perfect integration and that for any line bundle over a smooth variety $L \rightarrow X$, we have $z^{*}\left(z_{*}(1)\right)=c_{1}(L)$, where $z: X \hookrightarrow L$ is the zero section embedding.

For $K$-theory, the assignment $f \mapsto f_{*}$ is shown to be a perfect integration and the equation $z^{*}\left(z_{*}(1)\right)=$ $c_{1}(L)$ is verified in $[\mathrm{P} ; 2.6 .1]$. For morphic cohomology, we verify the axioms of a perfect integration by referring to results of $[\mathrm{FG}]$, [F3], and [F4]. Many of these results in the references cited are stated for Lawson homology but apply to morphic cohomology thanks to the natural duality isomorphism of [F3; 5.3]. Throughout this paragraph, let $A(-)=\bigoplus_{q, n} L^{q} H^{2 q+n}(-)$ and assume all variety are smooth, quasiprojective complex varieties. In reference to the axioms of $[\mathrm{P} ; 1.1 .3]$ (which define an "integration"), for $f$ proper, the fact that the map $f_{*}: A(Y) \rightarrow A(X)$ is a $A(X)$-module homomorphism (i.e., the projection formula holds) follows from $[\mathrm{F} 4 ; 5.4]$ and the fact that cap product coincides with cup product under the duality isomorphism (cf. [FW1; 3.5]). Axiom (1) (that $(f \circ g)_{*}=f_{*} \circ g_{*}$ holds for any pair of composable proper maps $f$ and $g$ ) is immediate from the definition and covariant functoriality of Lawson homology (cf. [F3; 3.1]). Axioms (2) (that push-forwards commute with pull-backs for transversal squares) follows from [FG; 3.4] and [F3; 4.3]. (We also use that any transversal square as in [P; 1.1.2] factors into transversal squares such that $\phi$ is either flat or a regular closed immersion.) To establish axiom (3) (that push-forwards along $X \times \mathbb{P}^{n} \rightarrow X$ commute with an arbitrary pull-back along $Y \rightarrow X$ ), first observe that the morphism $Y \rightarrow X$ can be factored into flat morphisms and regular closed immersions. The axiom is now seen to hold by applying [F4; 3.3], [FG; 3.4], [F3; 4.3], and the fact that flat pullback commutes with the duality isomorphisms (which is evident from the definitions). Axiom (4) is obvious from the definition of $f_{*}$. Axiom (5) (localization) follows from $[\mathrm{F} 3 ; 6.1]$. Thus, $f \mapsto f_{*}$ defines an integration. To show it is a perfect integration, we need to verify that a suitable form of the projective bundle formula holds (cf. $[\mathrm{P} ; 1.1 .6]$ ). First, we verify the normalization condition. Namely, let $L \rightarrow X$ be any line bundle and define $e(L)=z^{*}\left(z_{*}(1)\right) \in L^{1} H^{2}(X)$, where $z: X \hookrightarrow L$ is the zero section embedding. Under the isomorphism $L^{1} H^{2}(X) \cong C H^{2}(X)$, the element $e(L)$ corresponds to $z^{!}\left(z_{*}(1)\right)$, where $z^{!}$is the pull-back map on Chow groups for a regular closed immersion. Thus $e(L)=c_{1}(L)=D(L)$ (essentially by definition of $D(L)$ ). Finally, if $E$ is any vector bundle, define $\xi_{E}=e\left(\mathcal{O}_{E}(-1)\right) \in L^{1} H^{2}(\mathbb{P}(E))$. It remains to prove

$$
\left(\pi^{*}, \xi_{E} \cup \pi^{*}, \xi_{E}^{2} \cup \pi^{*}, \ldots, \xi_{E}^{r-1} \cup \pi^{*}\right): \bigoplus_{i=0}^{r-1} A(X) \rightarrow A(\mathbb{P}(E))
$$

is an isomorphism, where $\pi: \mathbb{P}(E) \rightarrow X$ is the canonical map. This follows from [F3; 6.5]

Remark 5.4. The reader can consult $[\mathrm{KW}]$ for a context in which a special case of Theorem 5.2 plays a central role.

The following result is another application of the rational isomorphism between semi-topological $K$-theory and morphic cohomology.

Theorem 5.5. Let $G$ be a finite group acting on the smooth connected variety $Y$ and assume that the quotient $Y / G=X$ is also smooth. Let $f: Y \rightarrow X$ be the quotient map, a finite (but not necessarily étale) map. Then the natural map

$$
f^{*}: K_{*}^{s s t}(X)_{\mathbb{Q}} \rightarrow K_{*}^{s s t}(Y)_{\mathbb{Q}}
$$

identifies $K_{*}^{s s t}(X)_{\mathbb{Q}}$ with the $G$-invariants of $K_{*}^{s s t}(Y)_{\mathbb{Q}}$.

Proof. By Theorem 4.7, it suffices to verify that the natural map on morphic cohomology

$$
f^{*}: L^{q} H^{m}(X, \mathbb{Q}) \rightarrow L^{q} H^{m}(Y, \mathbb{Q})^{G}
$$


is an isomorphism, for all $q, m$. Define

$$
f_{*}: \operatorname{Mor}\left(Y, \mathcal{C}_{0}\left(\mathbb{P}^{q}\right)\right)^{+} \rightarrow \mathcal{M o r}\left(X, \mathcal{C}_{0}\left(\mathbb{P}^{q}\right)\right)^{+}
$$

to be the continuous map induced by pushforward of cycles [F3; 3.1$]$ — that is, $f_{*}$ is the map induced by sending an integral subscheme $Z$ of $Y \times \mathbb{P}^{q}$ (that is finite over $Y$ ) to $\sum_{W}[k(Z): k(W)] W$, where the sum ranges over the irreducible components $W$ of the scheme theoretic image of $Z$ under $f \times i d$. Let $f_{*}$ also denote the induced map on morphic cohomology

$$
f_{*}: L^{q} H^{m}(Y) \rightarrow L^{q} H^{m}(X) .
$$

Then one may readily verify that $f_{*} \circ f^{*}$ coincides with multiplication by $|G|=\operatorname{deg}(f)$ on $L^{q} H^{m}(X)$, and we claim $f^{*} \circ f_{*}$ is the endomorphism of $L^{q} H^{m}(Y)$ sending $\gamma$ to $\sum_{g \in G} g^{*}(\gamma)$. Indeed, we claim this holds on the level of spaces - that is, the map

$$
f^{*} \circ f_{*}: \operatorname{Mor}\left(Y, \mathcal{C}_{0}\left(\mathbb{P}^{q}\right)\right)^{+} \rightarrow \mathcal{M o r}\left(Y, \mathcal{C}_{0}\left(\mathbb{P}^{q}\right)\right)^{+}
$$

is given by $\sum_{g \in G} g^{*}$. To show this, it suffices to restrict to the inverse image in $Y$ of the open dense subset $U$ of $X$ over which $f$ is étale - in other words, we may assume $f$ is étale, in which case the formula is obvious.

The result now follows, since the above formulas show $f^{*}$ is rationally injective and that the inclusion $L^{q} H^{m}(Y, \mathbb{Q})^{G} \hookrightarrow L^{q} H^{m}(Y, \mathbb{Q})$ is split by the map $\frac{1}{|G|} f^{*} \circ f_{*}$.

We next reformulate the Friedlander-Mazur $s$-operation in morphic cohomology.

Proposition 5.6. Let $s \in L^{1} H^{0}(\operatorname{Spec} \mathbb{C})$ denote the class represented by the element in

$$
\pi_{2}\left(\operatorname{Hom}\left(\Delta_{\text {top }}^{\bullet}, \mathcal{C}_{0}\left(\mathbb{P}^{1}\right)\right)^{+}\right) \cong \pi_{2}\left(Z_{0}\left(\mathbb{P}^{1}\right)\right) \cong \mathbb{Z}
$$

given by the map $S^{2} \cong\left(\mathbb{P}^{1}\right)^{\text {an }} \rightarrow Z_{0}\left(\mathbb{P}^{1}\right)$ sending $x \in \mathbb{P}^{1}$ to $x-\infty \in Z_{0}\left(\mathbb{P}^{1}\right)$. Then for any smooth variety $X$ of pure dimension $d$, there is a commutative square

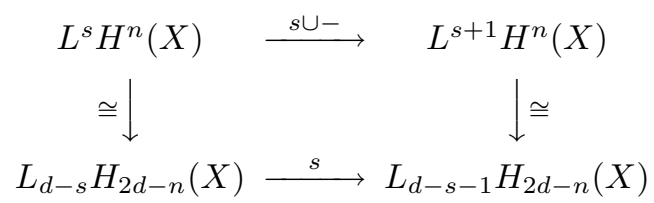

where the vertical maps are the duality isomorphisms of [FL2], [F3], the upper horizontal map is cup product with the class $s \in L^{1} H^{0}(\operatorname{Spec} \mathbb{C})$, and the lower horizontal map is the Friedlander-Mazur "s-operation" in Lawson homology [FM; §6].

Proof. The square (5.6.1) arises from the commutative square of spaces

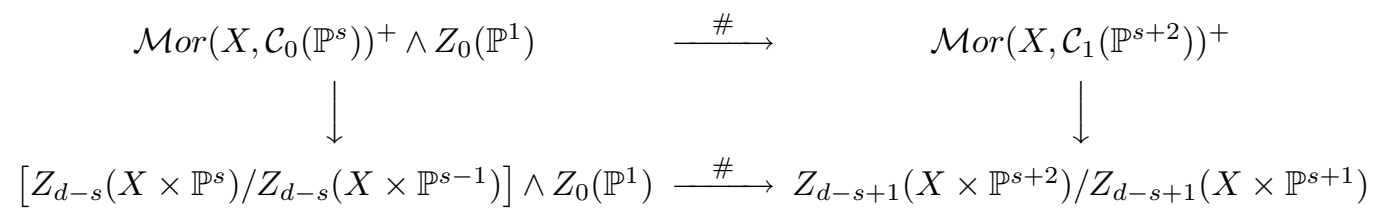

using the class $s$ in the evident manner. Here, the upper horizontal map is induced by $\left(f: X \rightarrow \mathcal{C}_{0}\left(\mathbb{P}^{s}\right), t \in\right.$ $\left.\mathcal{C}_{0}\left(\mathbb{P}^{1}\right)\right) \mapsto f \# t$ with $(f \# t)(x)=f(x) \# t \in \mathcal{C}_{1}\left(\mathbb{P}^{s+2}\right)$, the vertical maps are given by taking the graph of a 
map, and the lower horizontal map sends a pair of cycles to their join. (We have implicitly used an iterated form of the homotopy equivalence

$$
\pi^{*}: Z_{r}(X) \stackrel{\sim}{\longrightarrow} Z_{r+1}\left(X \times \mathbb{P}^{1}\right) / Z_{r+1}\left(X \times \mathbb{P}^{0}\right)
$$

discussed in the proof of [FG; 2.6].)

We define the Bott element

$$
\beta \in K_{2}^{s s t}(\operatorname{Spec} \mathbb{C}) \cong K_{\text {top }}^{-2}(p t) \cong \mathbb{Z}
$$

by the formula $c_{2,1}(\beta)=s$, where $s \in L^{1} H^{0}(\operatorname{Spec} \mathbb{C}) \cong \mathbb{Z}$ is given in Proposition 5.6. Note that $s$ is a generator for the abelian group $L^{1} H^{0}(\operatorname{Spec} \mathbb{C})$ and $c_{2,1}: K_{2}^{s s t}(\operatorname{Spec} \mathbb{C}) \rightarrow L^{1} H^{0}(\operatorname{Spec} \mathbb{C})$ is an isomorphism. In particular, $\beta$ is a generator of $K_{2}^{s s t}(\operatorname{Spec} \mathbb{C}) \cong \mathbb{Z}$, and, for any integer $n>0$, the image of $\beta$ under the boundary map

$$
K_{2}^{s s t}(\operatorname{Spec} \mathbb{C}) \rightarrow K_{1}^{s s t}(\operatorname{Spec} \mathbb{C}, \mathbb{Z} / n)
$$

in the evident long exact sequence is a generator of $K_{1}^{s s t}(\operatorname{Spec} \mathbb{C}, \mathbb{Z} / n) \cong \mathbb{Z} / n$. Since $K_{1}^{\text {sst }}(\operatorname{Spec} \mathbb{C}, \mathbb{Z} / n) \cong$ $K_{1}(\operatorname{Spec} \mathbb{C} ; \mathbb{Z} / n)$ by $[\mathrm{FW} 3 ; 3.7]$, we see that what we have referred to as the "Bott element" here corresponds with the usual Bott element in algebraic $K$-theory.

The following corollary asserts that multiplication by the Bott element in $K$-theory "covers" the FriedlanderMazur s-operation in morphic cohomology.

Corollary 5.7. For any quasi-projective complex variety $X$ and integer $j$, we have a commutative square

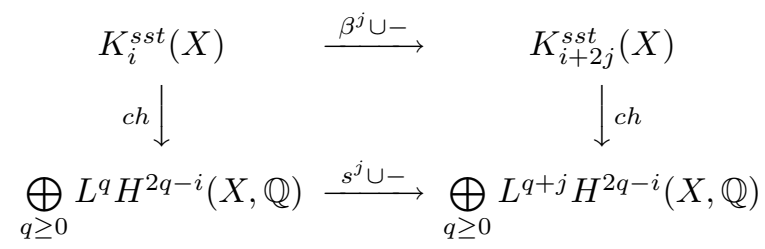

where the upper map is given by multiplication by the $j$-th power of the Bott element $\beta \in K_{2}^{\text {sst }}($ Spec $\mathbb{C})$ and the lower map is the $j$-th iterate of the (dual of the) Friedlander-Mazur s-operation.

Proof. Since the Chern classes $c_{2, q}$ for Spec $\mathbb{C}$ vanish for $q \neq 1$, we have $\operatorname{ch}_{2}(\beta)=s \in L^{1} H^{0}(\operatorname{Spec} \mathbb{C}, \mathbb{Q})$. The result follows immediately from the multiplicativity of the Chern character proven in Theorem 4.7.

Using Theorem 4.7 and Corollary 5.7, we deduce the following result. A version of this theorem was given in [FW2; 4.2] under the hypothesis that Theorem 4.4 of this paper was valid. Also, in [Wa], a version of this result is proved for smooth projective varieties. The groups $K_{t o p}^{n}\left(X^{a n}\right)$ for $n$ positive are defined in the usual manner by imposing Bott periodicity, so that $\bigoplus_{q \in \mathbb{Z}} K_{\text {top }}^{-q}\left(X^{a n}\right)=\left(\bigoplus_{q \geq 0} K_{\text {top }}^{-q}\left(X^{a n}\right)\right)\left[\frac{1}{\beta}\right]$.

Theorem 5.8. For any smooth, quasi-projective complex variety $X$, the natural map

$$
\mathcal{K}^{s s t}(X) \rightarrow \mathcal{K}_{\text {top }}\left(X^{a n}\right)
$$

induces an isomorphism on homotopy groups after inverting the Bott element $\beta \in K_{2}^{\text {sst }}(\operatorname{Spec} \mathbb{C})$ :

$$
\left(\bigoplus_{q \geq 0} K_{q}^{s s t}(X)\right)\left[\frac{1}{\beta}\right] \stackrel{\cong}{\longrightarrow} \bigoplus_{q \in \mathbb{Z}} K_{\text {top }}^{-q}\left(X^{a n}\right) .
$$


Proof. Recall that the Dold-Thom Theorem implies that Lawson homology of 0-cycles on $X$ is naturally isomorphic to Borel-Moore homology of $X^{\text {an }}$

$$
L_{0} H_{m}(X) \cong H_{m}^{B M}\left(X^{a n}\right) .
$$

Thus, duality relating morphic cohomology and Lawson homology implies that $L^{d} H^{m}(X) \cong H_{\text {sing }}^{m}\left(X^{a n}\right)$ where $d=\operatorname{dim} X$. Moreover, the natural isomorphisms

$$
L^{q} H^{m}(X) \cong \pi_{2 q-m} Z_{d}\left(X \times \mathbb{A}^{q}\right) \cong \pi_{2 q-m} Z_{0}\left(X \times \mathbb{A}^{q-d}\right) \cong H_{2 q-m}^{B M}\left(\left(X \times \mathbb{A}^{q-d}\right)^{a n}\right) \cong H_{2 d-m}^{B M}\left(X^{a n}\right)
$$

together with Poincare duality imply that the natural map

$$
L^{q} H^{m}(X) \rightarrow H_{\text {sing }}^{m}\left(X^{a n}\right)
$$

is an isomorphism for $q \geq d$. We conclude that the natural map

$$
\left(\bigoplus_{q} L^{q} H^{2 q-*}(X)\right)\left[\frac{1}{s}\right] \rightarrow \bigoplus_{q} H_{\text {sing }}^{2 q-*}\left(X^{a n}\right)
$$

is an isomorphism.

Corollary 5.7 thus enables us to conclude the rational version of the theorem; namely, the natural map

$$
\left(\bigoplus_{q \geq 0} K_{q}^{s s t}(X)\right)\left[\frac{1}{\beta}\right] \otimes \mathbb{Q} \rightarrow \bigoplus_{q \in \mathbb{Z}} K_{t o p}^{-q}\left(X^{a n}\right)_{\mathbb{Q}}
$$

is an isomorphism. The integral statement of the theorem now follows as in [FW2; 4.2] using Thomason's theorem [Th], asserting that mod- $n$ algebraic $K$-theory with the Bott element inverted is naturally isomorphic to mod- $n$ topological $K$-theory, and the natural isomorphism $K_{*}(X, \mathbb{Z} / n) \cong K_{*}^{s s t}(X, \mathbb{Z} / n)$ proven in [FW2].

We recall the "topological filtration" on singular cohomology defined by the formula

$$
T^{j} H_{\text {sing }}^{m}\left(X^{a n}, \mathbb{Z}\right)=\operatorname{im}\left(s^{j}: L^{d-j} H^{m}(X) \rightarrow L^{d} H^{m}(X) \cong H_{\text {sing }}^{m}\left(X^{a n}, \mathbb{Z}\right)\right),
$$

where $d=\operatorname{dim}(X)$. (We set $T^{j}=T^{0}$ if $j<0$.) This gives a filtration of the form

$$
H_{\text {sing }}^{m}\left(X^{a n}, \mathbb{Z}\right)=T^{0} H_{\text {sing }}^{m}\left(X^{a n}, \mathbb{Z}\right) \supset T^{1} H_{\text {sing }}^{m}\left(X^{a n}, \mathbb{Z}\right) \supset T^{2} H_{\text {sing }}^{m}\left(X^{a n}, \mathbb{Z}\right) \supset \cdots,
$$

with $T^{j} H_{\text {sing }}^{m}\left(X^{a n}, \mathbb{Z}\right)=0$ for $j>d$. Following Grothendieck, we define the coniveau filtration on $H_{\text {sing }}^{m}\left(X^{\text {an }}, \mathbb{Q}\right)$ by the formula

$$
\begin{aligned}
& G^{j} H_{\text {sing }}^{m}\left(X^{a n}, \mathbb{Q}\right)= \\
& \quad \sum\left\{\operatorname{ker}\left(H_{\text {sing }}^{m}\left(X^{a n}, \mathbb{Q}\right) \rightarrow H_{\text {sing }}^{m}\left((X-Y)^{a n}, \mathbb{Q}\right)\right) \quad \mid Y \subset X \text { closed of codimension at least } j\right\},
\end{aligned}
$$

giving a filtration of the form

$$
H_{\text {sing }}^{m}\left(X^{a n}, \mathbb{Z}\right)=G^{0} H_{\text {sing }}^{m}\left(X^{a n}, \mathbb{Z}\right) \supset G^{1} H_{\text {sing }}^{m}\left(X^{a n}, \mathbb{Z}\right) \supset G^{2} H_{\text {sing }}^{m}\left(X^{a n}, \mathbb{Z}\right) \supset \cdots
$$


For $X$ projective, $G^{j} H_{\text {sing }}^{m}\left(X^{a n}, \mathbb{Q}\right)$ is a subset of $T^{j} H_{\text {sing }}^{m}(X, \mathbb{Q})$ [FM; 4.3]. Moreover, in certain cases, equality of these two filtrations is known (cf. $[\mathrm{F} 2 ; 4.2])$. We also have the rational Hodge filtration

$$
H_{\text {sing }}^{m}\left(X^{a n}, \mathbb{Q}\right)=F^{0} H_{\text {sing }}^{m}\left(X^{a n}, \mathbb{Q}\right) \supset F^{1} H_{\text {sing }}^{m}\left(X^{a n}, \mathbb{Q}\right) \supset F^{2} H_{\text {sing }}^{m}\left(X^{a n}, \mathbb{Q}\right) \supset \cdots,
$$

where $F^{j} H_{\text {sing }}^{m}\left(X^{a n}, \mathbb{Q}\right)$ is defined by Grothendieck $[\mathrm{G}]$ to be the maximal sub-mixed Hodge structure of $H_{\text {sing }}^{m}\left(X^{a n}, \mathbb{Q}\right)$ contained in $H_{\text {sing }}^{m}\left(X^{a n}, \mathbb{Q}\right) \cap \bigoplus_{p \geq j} H_{\text {sing }}^{p, m-p}\left(X^{a n}, \mathbb{C}\right)$ (where $H_{\text {sing }}^{i, j}\left(X^{a n}, \mathbb{C}\right)$ refers to the standard Hodge decomposition of the complex cohomology of a complex manifold). Equivalently, $F^{j} H_{\text {sing }}^{m}\left(X^{a n}, \mathbb{Q}\right)$ is the maximal sub-mixed Hodge structure of $H_{\text {sing }}^{m}\left(X^{a n}, \mathbb{Q}\right)$ of span $m-2 j$ as defined (for homology) in [FM; 7.1].

As observed in $[\mathrm{FM}]$ in the dual context of homology, for any projective smooth variety and any $j$ there are natural inclusions

$$
T^{j} H_{\text {sing }}^{m}\left(X^{a n}, \mathbb{Q}\right) \subseteq G^{j} H_{\text {sing }}^{m}\left(X^{a n}, \mathbb{Q}\right) \subseteq F^{j} H_{\text {sing }}^{m}\left(X^{a n}, \mathbb{Q}\right)
$$

for all $j$.

Finally, we define a filtration on $K_{t o p}^{0}$ which is the evident analogue of the "topological filtration" on cohomology - namely, if $X$ has dimension $d$, set

$$
T^{j} K_{\text {top }}^{0}\left(X^{a n}\right)=\operatorname{im}\left(K_{2 d-2 j}^{s s t}(X) \stackrel{\beta^{j}}{\longrightarrow} K_{2 d}^{s s t}(X) \rightarrow K_{\text {top }}^{-2 d}\left(X^{a n}\right) \underset{\cong}{\stackrel{\beta^{d}}{\cong}} K_{\text {top }}^{0}\left(X^{a n}\right)\right) .
$$

(Again, $T^{j}=T^{0}$ for $j<0$.)

Theorem 5.10. For any smooth, quasi-projective complex variety $X$, the Chern character restricts to an isomorphism

$$
c h^{\text {top }}: T^{j} K_{\text {top }}^{0}\left(X^{a n}\right)_{\mathbb{Q}} \stackrel{\cong}{\longrightarrow} \bigoplus_{q \geq 0} T^{j-q} H_{\text {sing }}^{2 q}\left(X^{a n}, \mathbb{Q}\right),
$$

for all $j$. In other words, the weight $q$ piece of $T^{j} K_{\text {top }}^{0}\left(X^{a n}\right)_{\mathbb{Q}}$ is mapped isomorphically via the Chern character to $T^{j-q} H_{\text {sing }}^{2 q}\left(X^{a n}, \mathbb{Q}\right)$.

Proof. By Theorem 4.7 and Corollary 5.7, we have a commutative ladder of groups of the form

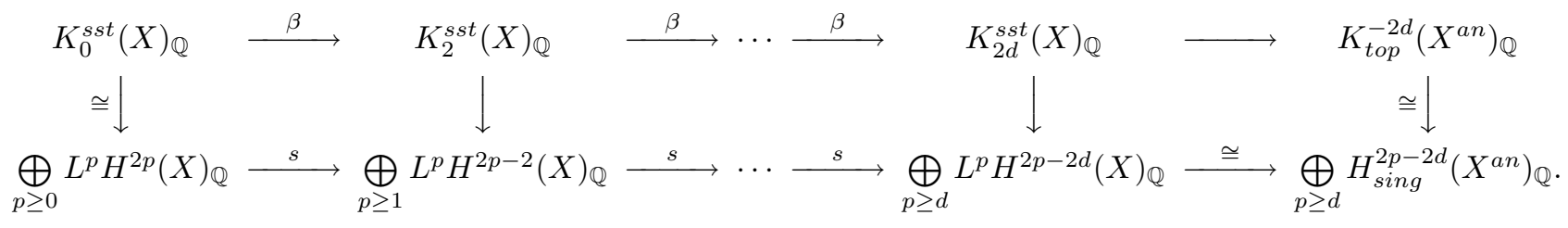

The horizontal maps increase the index of $L^{*}$ by one (except for the last map) and leave the cohomological index $H^{*}$ fixed. The vertical maps are defined by taking the composition of

$$
K_{2 i}^{s s t}(X)_{\mathbb{Q}} \stackrel{c h^{s s t}}{\cong} \bigoplus_{p \geq 0} L^{p} H^{2 p-2 i}(X)_{\mathbb{Q}} \rightarrow \bigoplus_{p \geq i} L^{p} H^{2 p-2 i}(X)_{\mathbb{Q}}, \quad 0 \leq i \leq d,
$$

where the second map is the canonical surjection. (If $L^{p} H^{m}(X)=0$ for $m<0$, as is conjectured, then all of the vertical maps would be isomorphisms.) This establishes the isomorphism

$$
c^{\text {top }}: T^{j} K_{\text {top }}^{0}\left(X^{a n}\right)_{\mathbb{Q}} \cong \operatorname{im}\left(s^{j}: \bigoplus_{p \geq d-j} L^{p} H^{2 p+2 j-2 d}(X)_{\mathbb{Q}} \rightarrow \bigoplus_{p \geq d} L^{p} H^{2 p-2 d}(X)_{\mathbb{Q}} \cong \bigoplus_{p \geq d} H_{\text {sing }}^{2 p-2 d}\left(X^{a n}\right)_{\mathbb{Q}}\right) \text {. }
$$


Observe that for any $m$ the maps

$$
L^{d} H^{m}(X)_{\mathbb{Q}} \rightarrow L^{d+1} H^{m}(X)_{\mathbb{Q}} \rightarrow \cdots \rightarrow H^{m}(X)_{\mathbb{Q}}
$$

are all isomorphisms and that, for each $d \geq p \geq d-j$, the map

$$
s^{j}: L^{p} H^{m}(X)_{\mathbb{Q}} \rightarrow L^{p+j} H^{m}(X)_{\mathbb{Q}} \cong H_{\text {sing }}^{m}\left(X^{a n}\right)_{\mathbb{Q}}
$$

factors as

$$
L^{p} H^{m}(X)_{\mathbb{Q}} \stackrel{s^{d-p}}{\longrightarrow} L^{d} H^{m}(X)_{\mathbb{Q}} \stackrel{s^{p+j-d}}{\cong} L^{p+j} H^{m}(X)_{\mathbb{Q}} \cong H_{\text {sing }}^{m}\left(X^{a n}\right)_{\mathbb{Q}} .
$$

The image of (5.10.2) therefore coincides with $T^{d-p} H_{\text {sing }}^{m}\left(X^{a n}\right)_{\mathbb{Q}}$ provided $p \geq d-j$. Using (5.10.1), we have that $c h^{\text {top }}$ maps $T^{j} K_{\text {top }}^{0}\left(X^{a n}\right)_{\mathbb{Q}}$ isomorphically to

$$
\begin{aligned}
& \operatorname{im}\left(s^{j}: \bigoplus_{p \geq d-j} L^{p} H^{2 p+2 j-2 d}(X)_{\mathbb{Q}} \rightarrow \bigoplus_{p \geq d} L^{p} H^{2 p-2 d}(X)_{\mathbb{Q}} \cong \bigoplus_{p \geq d} H_{\text {sing }}^{2 p-2 d}\left(X^{a n}\right)_{\mathbb{Q}}\right) \\
= & \bigoplus_{p \geq d-j} \operatorname{im}\left(s^{j}: L^{p} H^{2 p+2 j-2 d}(X)_{\mathbb{Q}} \rightarrow L^{p+j} H^{2 p+2 j-2 d}(X)_{\mathbb{Q}} \cong H_{\text {sing }}^{2 p+2 j-2 d}\left(X^{a n}\right)_{\mathbb{Q}}\right) \\
= & \bigoplus_{p \geq d-j} T^{d-p} H_{\text {sing }}^{2 p+2 j-2 d}\left(X^{a n}\right)_{\mathbb{Q}}=\bigoplus_{q \geq 0} T^{j-q} H_{\text {sing }}^{2 q}\left(X^{a n}\right)_{\mathbb{Q}} .
\end{aligned}
$$

We recall that S. Abdulali verifies that the topological filtration equals the rational Hodge filtration for any abelian variety which satisfies the Generalized Hodge Conjecture $[\mathrm{A}]$. The reader can consult $[\mathrm{A}]$ for a lengthy list of abelian varieties known to satisfy the generalized Hodge conjecture.

Theorem 5.11. Let $X$ be an abelian variety satisfying the Generalized Hodge Conjecture. Then there is a natural isomorphism

$$
T^{j} K_{\text {top }}^{0}\left(X^{a n}\right)_{\mathbb{Q}} \cong \bigoplus_{q \geq 0} F^{j-q} H_{\text {sing }}^{2 q}\left(X^{a n}, \mathbb{Q}\right) .
$$

Proof. This follows immediately from Theorem 5.10 and Abdulali's identity

$$
T^{j} H_{\text {sing }}^{n}\left(X^{a n}, \mathbb{Q}\right)=F^{j} H_{\text {sing }}^{n}\left(X^{a n}, \mathbb{Q}\right)
$$

valid for abelian varieties satisfying the Generalized Hodge Conjecture.

\section{REFERENCES}

[A] S. Abdulali, Filtrations on the cohomology of abelian varieties, CRM Proceedings and Lecture Notes 24 (2000), $3-12$.

$[\mathrm{AH}] \quad$ M. Atiyah and F. Hirzebruch, Vector bundles and homogeneous spaces, Proc. Sympos. Pure Math. Vol. III, 7-38.

[BFM] P. Baum, W. Fulton, and R. MacPherson, Riemann-Roch for singular varieties, Publ. Math. IHES 45 (1975), $101-145$.

[B] S. Bloch, Algebraic cycles and higher K-theory, Adv in Math 61 (3) (1986), 267-304.

[BL] S. Bloch and S. Lichtenbaum, A spectral sequence for motivic cohomology, Invent. Math. (to appear).

[BS] A. Borel and J. P. Serre, Le théorème de Riemann-Roch, Bull. Soc. Math. France 86 (1958), 97-136.

$[\mathrm{BF}] \quad$ A. K. Bousfield and E. Friedlander, Homotopy theory of $\Gamma$-spaces, spectra, and bisimplicial sets, Lecture Notes in Math 658, 80-130. 
[CL1] R. L. Cohen and P. Lima-Filho, An algebraic geometric realization of the Chern character, Preprint, available at http://www.math.uiuc.edu/K-theory/379/.

[CL2] R. L. Cohen and P. Lima-Filho, Holomorphic K-theory, algebraic co-cycles, and loop groups., K-theory 23 (2001), $345-376$.

[DW] B. H. Dayton and C. A. Weibel, A spectral sequence for the $K$-theory of affine glued schemes, Algebraic $K$-theory, Evanston 1980, Springer Lecture Notes in Math., vol. 854, 1981, pp. 24-92.

[DeJ] A. J. de Jong, Smoothness, semi-stability and alterations, Inst. Hautes Études Sci Publ. Math, 83 (1996), 51-93..

[D] P. Deligne, Théorie de Hodge III, Inst. Hautes Études Sci Publ. Math, 44 (1974), 5-77..

[F1] E. Friedlander, Algebraic coycles, Chow varieties, and Lawson homology, Comp. Math. 77 (1991), 55-93.

[F2] E. Friedlander, Filtrations on algebraic cycles and homology., Ann. Sci. École Norm. Sup. 28 (4) (1995), $317-343$.

[F3] E. Friedlander, Algebraic cocycles on normal, quasi-projective varieties, Comp. Math. 110 (1998), $127-162$.

[F4] E. Friedlander, Bloch-Ogus properties for topological cycle theory, Ann. Sci. Ec. Norm. Sup 44 (2000), 57-79.

[FG] E. Friedlander and O. Gabber, Cycle spaces and intersection theory, Topological Methods in Modern Mathematics, 1993, pp. 325-370.

[FHW] E. Friedlander, C. Haesemeyer, and M. Walker, Comparing K-theory spectral sequences (in preparation).

[FL1] E. Friedlander and H. B. Lawson, A theory of algebraic cocycles, Annals of Math. 136 (1992), 361-428.

[FL2] E. Friedlander and H. B. Lawson, Duality relating spaces of algebraic cocycles and cycles, Topology 36 (1997), 533-565.

[FM] E. Friedlander and B. Mazur, Filtrations on the Homology of Algebraic Varieties, Memoirs of the AMS, Number $529,1991$.

[FS] E. Friedlander and A. Suslin, The spectral sequence relating algebraic K-theory to motivic cohomology, Preprint, available at http://www.math.uiuc.edu/K-theory/432/.

[FV] E. Friedlander and V. Voevodsky, Bivariant cycle cohomology, Cycles, Transfers, and Motivic Homology Theories, Annals of Math. Studies, 2000.

[FW1] E. Friedlander and M. Walker, Function spaces and continuous algebraic pairings for varieties, Comp. Math. 125 (2001), 69-110.

[FW2] E. Friedlander and M. Walker, Semi-topological K-theory using function complexes, Topology 41 (2002), 591-644.

[FW3] E. Friedlander and M. Walker, Comparing K-theories for complex varieties, Amer. J. Math. 123 (2001), 779-810.

[FW4] E. Friedlander and M. Walker, Semi-topological K-theory of real varieties., Proceedings of the International Colloquium on Algebra, Arithmetic and Geometry, Tata Institute of Fundamental, Research, Mumbai 2000, (2001), $219-326$.

[Fu] W. Fulton, Intersection Theory, 1984.

[Gr] D. Grayson, Adams operations on higher K-theory, K-Theory 6, 97-111.

[GW] D. Grayson and M. Walker, Geometric models for algebraic K-theory, K-theory 20 (4) (2000), 311-330.

[G] A. Grothendieck, Hodge's general conjecture is false for trivial reasons, Topology 8 (1969), 299-303.

$[\mathrm{H}] \quad$ H. Hironaka, Resolution of singularities of an algebraic variety over a field of characteristic zero. I. , II., Annals of Math. (2) 79 (1964), 205-326.

[KW] M. Karoubi and C. Weibel, Algebraic and Real K-theory of real varieties, Topology (to appear).

[J] J. P. Jouanolou, Une suite exacte de Mayer-Vietoris en K-théorie algébrique, Algebraic K-theory I: Higher algebraic $K$-theories, vol. 341, Springer Lecture Notes Math, 1973, pp. 293-316.

[L] H. Blaine Lawson, Algebraic cycles and homotopy theory, Ann. of Math. (2) 129 (1989), 253-291.

[Le] M. Levine, Bloch's higher Chow groups revisited, Astérisque 226 (1994), 235-320.

[Ma] J. P. May, The geometry of iterated loop spaces, vol. 271, Lecture Notes Math., 1972.

[P] I. Panin, Riemann-Roch Theorem for oriented cohomology, Preprint, available at http://www.math.uiuc.edu/Ktheory $/ 552 /$.

[PS] I. Panin and A. Smirnov, Oriented cohomology theories and Riemann-Roch-Grothendieck theorem, Preprint, available at http://www.math.uiuc.edu/K-theory/459/.

[Q] D. Quillen, Higher algebraic K-theory. I, Algebraic $K$-theory, I: Higher $K$-theories (Proc. Conf., Battelle Memorial Inst., Seattle, Wash., 1972), Springer-Verlag, L.N.M 341, 1973, pp. 85-147.

[SGA4] M. Artin, A. Grothendieck, and J.-L. Verdier, Theorie des Topos et Cohomologie Etale des Schemas, vol. 270, Lecture Notes Math., 1972.

[SGA6] P. Berthelot, A. Grothendieck, and L. Illusie, Théorie des Intersections et Thérème de Riemann-Roch, vol. 225, Lecture Notes Math., 1971.

[Su] A. Suslin, Higher Chow groups and étale cohomology, Cycles, transfers, and motivic homology theories, Annals of Math. Studies, vol. 143, Princeton Univ. Press, Princeton, NJ, 2000, pp. 239-254.

[SV1] A. Suslin and V. Voevodsky, Singular homology of abstract algebraic varieties, Invent. Math. 123 (1996), 61-94.

[SV2] A. Suslin and V. Voevodsky, Relative cycles and Chow sheaves, Cycles, transfers, and motivic homology theories, Annals of Math. Studies, vol. 143, Princeton Univ. Press, Princeton, NJ, 2000, pp. 10-86. 
[Th] R. Thomason, Algebraic K-theory and tale cohomology, Ann. Sci. École Norm. Sup. 18 (1985), 437-552.

[TT] R. Thomason and T. Trobaugh, Higher algebraic K-theory ofschemes and of derived categories., The Grothendieck Festschrift, III, Progr. Math., vol. 88, Birkhäuser, 1990, pp. 247-435.

[V1] V. Voevodsky, Homology of schemes, Selecta Mathematica 2 (1996), 111-153.

[V2] V. Voevodsky, Cohomological theory of presheaves with transfers, Cycles, transfers, and motivic homology theories, Annals of Math. Studies, vol. 143, Princeton Univ. Press, Princeton, NJ, 2000, pp. 10-86.

[Wa] M. Walker, Semi-topological K-homology and Thomason's Theorem, Preprint, available at http://www.math.uiuc.edu/Ktheory $/ 497 /$.

[We] C. Weibel, Homotopy algebraic K-theory, Contemp. Math. 83, Amer. Math. Soc., Providence, RI, 461-488.

Department of Mathematics, Northwestern University, Evanston, IL, 60208-2730, U.S.A.

E-mail address: eric@math.northwestern.edu

Department of Mathematics and Statistics, University of Nebraska - Lincoln, Lincoln, Ne, 68588-0323, U.S.A.

E-mail address: mwalker@math.unl.edu 